DOI 10.4171/JEMS/333

Andrea R. Nahmod · Tadahiro Oh · Luc Rey-Bellet · Gigliola Staffilani

\title{
Invariant weighted Wiener measures and almost sure global well-posedness for the periodic derivative NLS
}

Received January 6, 2011

\begin{abstract}
We construct an invariant weighted Wiener measure associated to the periodic derivative nonlinear Schrödinger equation in one dimension and establish global well-posedness for data living in its support. In particular almost surely for data in a Fourier-Lebesgue space $\mathcal{F} L^{s, r}(\mathbb{T})$ with $s \geq 1 / 2,2<r<4,(s-1) r<-1$ and scaling like $H^{1 / 2-\epsilon}(\mathbb{T})$, for small $\epsilon>0$. We also show the invariance of this measure.
\end{abstract}

\section{Introduction}

In the past few years, methods such as those by J. Bourgain (high-low method, e.g. [5, 6]) on the one hand and by J. Colliander, M. Keel, G. Staffilani, H. Takaoka and T. Tao (I-method or method of almost conservation laws, e.g. $[15,16,17])$ on the other, have been applied to study the global in time existence for dispersive equations at regularities which are right below or in between those corresponding to conserved quantities. It turns out, however, that for many dispersive equations and systems there still remains a gap between the local in time results and those that could be globally achieved. In those cases, it seems natural to return to one of Bourgain's early approaches for periodic dispersive equations (NLS, KdV, mKdV, Zakharov system) [3, 4, 5, 7, 8, 9] where global in time existence was studied in the almost sure sense via the existence and invariance of the associated Gibbs measure (cf. Lebowitz, Rose and Speer's and Zhidkov's works [30], [48]). More recently this approach has been used for example by N. Tzvetkov [44, 45] for subquintic radial nonlinear wave equation on the disc, N. Burq and N. Tzvetkov [12, 13] for subcubic and subquartic radial nonlinear wave equations on $3 \mathrm{~d}$ ball, N. Burq,

A. R. Nahmod: Radcliffe Institute for Advanced Study, Harvard University, Byerly Hall, 8 Garden Street, Cambridge, MA 02138, USA, and Department of Mathematics, University of Massachusetts, 710 N. Pleasant Street, Amherst, MA 01003, USA; e-mail: nahmod@math.umass.edu

T. Oh: Department of Mathematics, 40 St. George Street, Toronto, Ontario, Canada M5S 2E4, USA; e-mail: oh@math.toronto.edu

L. Rey-Bellet: Department of Mathematics, University of Massachusetts, 710 N. Pleasant Street, Amherst, MA 01003, USA; e-mail: luc@math.umass.edu

G. Staffilani: Radcliffe Institute for Advanced Study, Harvard University, Byerly Hall, 8 Garden Street, Cambridge, MA 02138, USA, and Department of Mathematics, Massachusetts Institute of Technology, 77 Massachusetts Avenue, Cambridge, MA 02139, USA;

e-mail: gigliola@math.mit.edu 
L. Thomann, and N. Tzvetkov [11] for the nonlinear Schrödinger equation with harmonic potential, and by T. Oh [33, 34, 35, 36] for the periodic KdV-type coupled systems, Schrödinger-Benjamin-Ono system and white noise for the KdV equation.

Failure to show global existence by Bourgain's high-low method or the I-method might come from certain 'exceptional' initial data set, and the virtue of the Gibbs measure is that it does not see that exceptional set. At the same time, the invariance of the Gibbs measure, just like the usual conserved quantities, can be used to control the growth in time of those solutions in its support and extend the local in time solutions to global ones almost surely. The difficulty in this approach lies in the actual construction of the associated Gibbs measure and in showing both its invariance under the flow and the almost sure global well-posedness, since, on the one hand, we need invariance to show global well-posedness, and on the other hand we need globally defined flow to discuss invariance.

Our goal in this paper is to construct an invariant weighted Wiener measure associated to the periodic derivative nonlinear Schrödinger equation DNLS in (2.1) in one dimension and establish global well-posedness for data living in its support. In particular almost surely for data in a Fourier-Lebesgue space $\mathcal{F} L^{s, r}$ defined in (2.2) below (cf. [27, 21, 14, 22]) and scaling like $H^{1 / 2-\epsilon}(\mathbb{T})$, for small $\epsilon>0$. The motivation for this paper stems from the fact that by scaling DNLS should be well-posed for data in $H^{\sigma}, \sigma \geq 0$, but the results obtained so far are much weaker.

Local well-posedness is known for $\sigma \geq 1 / 2$ for the nonperiodic [40] and periodic [26] cases while global well-posedness is known for $\sigma \geq 1 / 2$ for the nonperiodic case ( $\sigma>1 / 2$ in [16] and $\sigma \geq 1 / 2$ in [31]) and for $\sigma>1 / 2$ in the periodic case [47]. Furthermore, in the nonperiodic case the Cauchy initial value problem for DNLS is ill-posed for data in $H^{\sigma}(\mathbb{R}), \sigma<1 / 2$ [40], [2], a strong indication that ill-posedness should also be expected in the periodic case in that range. Grünrock and Herr [22] have recently established local well-posedness for the periodic DNLS in Fourier-Lebesgue spaces $\mathcal{F} L^{s, r}$, which for appropriate choices of $(s, r)$ scale like $H^{\sigma}(\mathbb{T})$ for any $\sigma>1 / 4$. Their result is the starting point of this work (cf. Section 2 for a more detailed discussion).

The measure we construct is based on the energy functional rather than the Hamiltonian. Hence we simply refer to it as weighted Wiener measure rather than Gibbs measure since the name 'Gibbs measure' has traditionally been reserved for those weighted Wiener measures constructed using the Hamiltonian. By invariance of a measure $\mu$ we mean that if $\Phi(t)$ denotes the flow map associated to our nonlinear equation then $\Phi(t)$ is defined for all $t \in \mathbb{R}, \mu$-almost surely and for all $f \in L^{1}(\mu)$ and all $t \in \mathbb{R}$,

$$
\int f(\Phi(t)(\phi)) \mu(d \phi)=\int f(\phi) \mu(d \phi) .
$$

In general terms our aim is to construct a well defined measure $\mu$ so that local wellposedness of the periodic DNLS holds in some space $\mathcal{B}$ containing the support of $\mu$. Then we show almost sure global well-posedness as well as the invariance of $\mu$ via a combination of the methods of Bourgain and Zhidkov [48] in the context of NLS, KdV, mKdV. In implementing this scheme however we need to overcome two main obstacles due to the need to gauge the equation to show local well-posedness (e.g. [40, 26]) and to construct 
an invariant measure. The symplectic form associated to the periodic gauged derivative nonlinear Schrödinger equation GDNLS in (2.6) does not commute with Fourier modes truncation and so the truncated finite-dimensional systems are not necessarily Hamiltonian. The first (mild) obstacle is to show the conservation of the Lebesgue measure associated to the finite-dimensional approximation to the periodic gauged derivative nonlinear Schrödinger equation FGDNLS, defined in (3.1) by hand, rather than by using the Hamiltonian structure. The second obstacle is much more serious and is at the heart of this work. The energy $\mathcal{E}$ defined in (2.13) associated to the gauged periodic DNLS ${ }^{1}$ which we prove to be conserved in time, ceases to be so when computed on solutions of the finite-dimensional approximation equation, that is, $\frac{d}{d t} \mathcal{E}\left(v^{N}\right) \neq 0$, when $v^{N}$ is a solution to the finite-dimensional gauged DNLS (see (4.9)). In other words the finite-dimensional weighted Wiener measure is not invariant any longer and unlike in Zhidkov's work [48] on $\mathrm{KdV}$ we do not have a priori knowledge of global well-posedness. We show however that it is almost invariant in the sense that we can control the growth in time of $\mathcal{E}\left(v^{N}\right)(t)$. This idea is reminiscent of the I-method. However, while in the I-method one needs to estimate the variation of the energy of solutions to the infinite-dimensional equation at time $t$ smoothly projected onto frequencies of size up to $N$, here one needs to control the variation of the energy $\mathcal{E}$ of the solution $v^{N}$ to the finite-dimensional approximation equation FGDNLS. We note that the loss in energy conservation for solutions to the finite-dimensional equation is principally due to the manner one chooses to approximate the infinite-dimensional gauged equation by using Fourier projections onto the first $N$ th frequencies. In [3] Bourgain describes an alternative approach that relies on using a discrete system of ODE which seems to preserve the conservation of energy. This approach however entails a number of other difficulties, for one needs to replace the circle $\mathbb{T}$ by the cyclic group $\mathbb{Z}_{N}$ and carry out the analysis on cyclic groups. We choose not to follow this path here.

We expect the ungauged invariant Wiener measure associated to DNLS (2.1) we obtain in Section 7 to be absolutely continuous with respect to the weighted Wiener measure constructed by Thomann and Tzvetkov [42]. This question is addressed in a forthcoming paper [32].

The paper is organized as follows. In Section 2 we present some general background, notation and results on the derivative nonlinear Schrödinger equation in one dimension. In Section 3 we discuss FGDNLS. In Section 4 we overcome the first two obstacles mentioned above. Namely we prove the invariance of the Lebesgue measure associated to FGDNLS and devote the rest of the section to prove our energy growth estimate Theorem 4.2. In Section 5 we carry out the construction of the weighted Wiener measure associated to the GDNLS. In Section 6 we prove the almost sure global well-posedness result for the GDNLS and the invariance of the measure constructed in section 5. Finally in Section 7 we translate back our results to the ungauged DNLS equation.

Notation. Whenever we write $a+$ for $a \in \mathbb{R}$ we mean $a+\varepsilon$ for some $\varepsilon>0$; similarly for $a$-. In addition, we write $A \lesssim B$ to mean there exists some absolute constant $C>0$ such that $A \leq C B$.

\footnotetext{
1 We emphasize $\mathcal{E}$ is not the Hamiltonian of the gauged DNLS.
} 


\section{The derivative NLS equation in one dimension}

The initial value problem for DNLS takes the form

$$
\left\{\begin{array}{l}
u_{t}-i u_{x x}=\lambda\left(|u|^{2} u\right)_{x} \\
\left.u\right|_{t=0}=u_{0}
\end{array}\right.
$$

where either $(x, t) \in \mathbb{R} \times(-T, T)$ or $(x, t) \in \mathbb{T} \times(-T, T)$ and $\lambda$ is real. In this paper we will take $\lambda=1$ for convenience. DNLS is a Hamiltonian PDE whose flow conserves also mass and energy, i.e. the following quantities are conserved in time ${ }^{2}$ (cf. $\left.[28,25,26]\right)$ :

$$
\begin{aligned}
\text { mass: } \quad M(u)(t) & =\int|u(x, t)|^{2} d x, \\
\text { energy: } \quad E(u)(t) & =\int\left|u_{x}\right|^{2} d x+\frac{3}{2} \operatorname{Im} \int u^{2} \bar{u} \overline{u_{x}} d x+\frac{1}{2} \int|u|^{6} d x, \\
\text { hamiltonian: } \quad H(u)(t) & =\operatorname{Im} \int u \overline{u_{x}} d x+\frac{1}{2} \int|u|^{4} d x .
\end{aligned}
$$

DNLS was introduced as a model for the propagation of circularly polarized Alfvén waves in a magnetized plasma with a constant magnetic field (cf. Sulem-Sulem [39]). The equation is scale invariant for data in $L^{2}$, i.e. if $u(x, t)$ is a solution then $u_{a}(x, t)=$ $a^{\alpha} u\left(a x, a^{2} t\right)$ is also a solution if and only if $\alpha=1 / 2$. Thus a priori one expects some form of existence and uniqueness results for (2.1) for data in $H^{\sigma}, \sigma \geq 0$. Many results are known for the Cauchy problem with smooth data, including data in $H^{1}$, such as those by M. Tsutsumi and I. Fukuda [43], N.Hayashi [23], N. Hayashi and T. Ozawa [24, 25] and T. Ozawa [37] and others (cf. references therein).

In looking for solutions to (2.1) we face a derivative loss arising from the nonlinear term $\left(|u|^{2} u\right)_{x}=u^{2} \bar{u}_{x}+2|u|^{2} u_{x}$ and hence for low regularity data the key is to somehow make up for this loss.

For the nonperiodic case $(x \in \mathbb{R})$ Takaoka [40] proved sharp local well-posedness (LWP) in $H^{1 / 2}(\mathbb{R})$ relying on the gauge transformation used by Hayashi and Ozawa [24, 25] and the so-called Fourier restriction norm method. Then, Colliander-Keel-StaffilaniTakaoka and Tao $[15,16]$ established global well-posedness (GWP) for data in $H^{\sigma}(\mathbb{R})$, $\sigma>1 / 2$, of small $L^{2}$ norm using the so-called I-method on the gauge equivalent equation (see also [41]). Here, small in $L^{2}$ just means less than an appropriate constant $\sqrt{2 \pi / \lambda}$ which forces the associated 'energy' to be positive via the Gagliardo-Nirenberg inequality. This result was recently improved by Miao, Wu and $\mathrm{Xu}$ to $\sigma \geq 1 / 2$. The Cauchy initial value problem for DNLS is ill-posed for data in $H^{\sigma}$ and $\sigma<1 / 2$ (data map fails to be $C^{3}$ or uniformly $C^{0}$ [40], [2]). In [21] A. Grünrock proved that the nonperiodic DNLS is locally well-posed in the Fourier-Lebesgue spaces $\mathcal{F} L^{s, r}(\mathbb{R})$ which for appropriate choices of $(s, r)$ scale like $H^{\sigma}(\mathbb{R})$ for any $\sigma>0$ (cf. (2.2) below).

In the periodic setting, S. Herr [26] showed that the Cauchy problem associated to periodic DNLS is locally well-posed for initial data $u(0) \in H^{\sigma}(\mathbb{T}), \sigma \geq 1 / 2$, in the sense

\footnotetext{
2 In fact, DNLS is completely integrable.
} 
of local existence, uniqueness and continuity of the flow map. Herr's proof is based on an adaptation to the periodic setting of the gauge transformation introduced by Hayashi [23] and Hayashi and Ozawa [24, 25] on $\mathbb{R}$, in conjunction with sharp multilinear estimates for the gauged equivalent equation in periodic Fourier restriction norm spaces $X^{s, b}$ that yield local well-posedness for the gauged equation. Moreover, by use of conservation laws, the problem is also shown to be globally well-posed for $\sigma \geq 1$ and data which is small in $L^{2}$ (as in [15, 16]) [26]. More recently, Win [47] applied the I-method to prove GWP in $H^{\sigma}(\mathbb{T})$ for $\sigma>1 / 2$.

A. Grünrock and S. Herr [22] showed that the Cauchy problem associated to DNLS is locally well-posed for initial data $u_{0} \in \mathcal{F} L^{s, r}(\mathbb{T})$ with $2<r<4$ and $s \geq 1 / 2$ where

$$
\left\|u_{0}\right\|_{\mathcal{F} L^{s, r}(\mathbb{T})}:=\left\|\langle n\rangle^{s} \widehat{u}_{0}\right\|_{\ell_{n}^{r}(\mathbb{Z})} .
$$

These spaces scale like the Sobolev $H^{\sigma}(\mathbb{T})$ ones where $\sigma=s+1 / r-1 / 2$. The proof is based on Herr's adapted periodic gauge transformation and new multilinear estimates for the gauged equivalent equation in an appropriate variant of Fourier restriction norm spaces $X_{r, q}^{s, b}$ introduced by Grünrock-Herr [22]. ${ }^{3}$

For $s, b \in \mathbb{R}, r, q \geq 1$ we define the space $X_{r, q}^{s, b}$ as the completion of the Schwartz space $\mathcal{S}(\mathbb{T} \times \mathbb{R})$ with respect to the norm

$$
\|u\|_{X_{r, q}^{s, b}}:=\left\|\langle n\rangle^{s}\left\langle\tau+n^{2}\right\rangle^{b} \widehat{u}(n, \tau)\right\|_{\ell_{n}^{r} L_{\tau}^{q}}
$$

where first we take the $L_{\tau}^{q}$ norm and then the $\ell_{n}^{r}$ one. We also define the space

$$
\|u\|_{X_{r, q ;-}^{s, b}}:=\left\|\langle n\rangle^{s}\left\langle\tau-n^{2}\right\rangle^{b} \widehat{u}(n, \tau)\right\|_{\ell_{n}^{r} L_{\tau}^{q},}
$$

and note that $u \in X_{r, q}^{s, b}$ if and only if $\bar{u} \in X_{r, q ;-}^{s, b}$.

For $\delta>0$ fixed, we define the restriction space $X_{r, q}^{s, b}(\delta)$ of all $v=\left.u\right|_{[-\delta, \delta]}$ for some $u \in X_{r, q}^{s, b}$ with norm

$$
\|v\|_{X_{r, q}^{s, b}(\delta)}:=\inf \left\{\|u\|_{X_{r, q}^{s, b}}: u \in X_{r, q}^{s, b} \text { and } v=\left.u\right|_{[-\delta, \delta]}\right\} .
$$

When we take $q=2$ we simply write $X_{r, 2}^{s, b}=X_{r}^{s, b}$. Note $X_{2,2}^{s, b}=X^{s, b}$. Later we will also use the space

$$
Z_{r}^{s}(\delta):=X_{r, 2}^{s, 1 / 2}(\delta) \cap X_{r, 1}^{s, 0}(\delta) .
$$

Some simple embeddings are as follows. For $s, b_{1}, b_{2} \in \mathbb{R}, r \geq 1$ and $b_{1}>b_{2}+1 / 2$,

$$
X_{r, 2}^{s, b_{1}} \subset X_{r, 1}^{s, b_{2}} \quad \text { and } \quad X_{r, 1}^{s, 0} \subset C\left(\mathbb{R}, \mathcal{F} L^{s, r}\right)
$$

which follow by Cauchy-Schwarz with respect to the $L_{\tau}^{1}$ norm and by $\mathcal{F}^{-1} L^{1} \subset L^{\infty}$ respectively. In particular

$$
Z_{r}^{s}(\delta) \subset C\left([-\delta, \delta], \mathcal{F} L^{s, r}\right)
$$

\footnotetext{
3 Note that in our notation the indices $(r, q)$ are the dual of the corresponding ones in GrünrockHerr [22].
} 

below.

We finally recall the following estimate ${ }^{4}$ heavily used in the proof of Theorem 4.2

Lemma 2.1 ([22, Lemma 5.1]). Let $1 / 3<b<1 / 2$ and $s>3(1 / 2-b)$. Then

$$
\|u v \bar{w}\|_{L_{x t}^{2}} \lesssim\|u\|_{X^{s, b}}\|v\|_{X^{s, b}}\|w\|_{X^{0, b}} .
$$

In particular if $b=(1 / 2)-$, then

$$
\|u v \bar{w}\|_{L_{x t}^{2}} \lesssim\|u\|_{X^{\epsilon, \frac{1}{2}-}}\|v\|_{X^{\epsilon, \frac{1}{2}-}}\|w\|_{X^{0, \frac{1}{2}-}},
$$

for small $\epsilon>0$; while when $b=(1 / 3)+$,

$$
\|u v \bar{w}\|_{L_{x t}^{2}} \lesssim\|u\|_{X^{\frac{1}{2}-, \frac{1}{3}+}}\|v\|_{X^{\frac{1}{2}-, \frac{1}{3}+}}\|w\|_{X^{0, \frac{1}{3}+}} \cdot
$$

\subsection{The periodic gauged derivative NLS equation}

We first recall S. Herr's gauge transformation. For $f \in L^{2}(\mathbb{T})$, let

$$
G(f)(x):=\exp (-i J(f)) f(x)
$$

where

$$
J(f)(x):=\frac{1}{2 \pi} \int_{0}^{2 \pi} \int_{\theta}^{x}\left(|f(y)|^{2}-\frac{1}{2 \pi}\|f\|_{L^{2}(\mathbb{T})}^{2}\right) d y d \theta .
$$

Note $G(f)$ is $2 \pi$-periodic since its integrand has zero mean value. Then for $u \in$ $C\left([-T, T] ; L^{2}(\mathbb{T})\right)$ and $m(u):=\frac{1}{2 \pi} \int_{\mathbb{T}}|u(x, 0)|^{2} d x$ the adapted periodic gauge is defined as ${ }^{5}$

$$
\mathcal{G}(u)(t, x):=G(u(t))(x-2 \operatorname{tm}(u)) .
$$

Note the $L^{2}$ norm of $\mathcal{G}(u)(t, x)$ is still conserved since the torus is invariant under translation. The map

$$
\mathcal{G}: C\left([-T, T] ; H^{\sigma}(\mathbb{T})\right) \rightarrow C\left([-T, T] ; H^{\sigma}(\mathbb{T})\right)
$$

is a homeomorphism for any $\sigma \geq 0$ and locally bi-Lipschitz on subsets of $C\left([-T, T] ; H^{\sigma}(\mathbb{T})\right)$ with prescribed $\|u(0)\|_{L^{2}}([26])$. Moreover the same is true if we replace $H^{\sigma}(\mathbb{T})$ by $\mathcal{F} L^{s, r}$ with $s>1 / 2-1 / r$ when $2<r<\infty$ and $s \geq 0$ when $r=2$ ([22]).

Then if $u$ is a solution to DNLS $(2.1)$ and $v:=\mathcal{G}(u)$ we see that $v$ solves the gauged DNLS equation (GDNLS)

$$
v_{t}-i v_{x x}=-v^{2} \bar{v}_{x}+\frac{i}{2}|v|^{4} v-i \psi(v) v-i m(v)|v|^{2} v
$$

\footnotetext{
4 This is a trilinear refinement of Bourgain's $L^{6}(\mathbb{T})$ Strichartz estimate [10].

5 Recall $m(u)(t)$ is conserved under the flow of (2.1).
} 
with initial data $v(0)=\mathcal{G}(u(0))$, where

$$
\begin{aligned}
& m(v)(t):=\frac{1}{2 \pi} \int_{\mathbb{T}}|v(x, t)|^{2} d x, \\
& \psi(v)(t):=-\frac{1}{\pi} \int_{\mathbb{T}} \operatorname{Im}\left(v \overline{v_{x}}\right) d x+\frac{1}{4 \pi} \int_{\mathbb{T}}|v|^{4} d x-m(v)^{2} .
\end{aligned}
$$

Note that $m(v)$ is conserved in time, more precisely $m(v)(t)=\frac{1}{2 \pi} \int_{\mathbb{T}}|v(x, 0)|^{2} d x=$ $m(u)$, and both $m(v)$ and $\psi(v)$ are real.

The initial value problem associated to (2.6) with data in $\mathcal{F} L^{s, r}(\mathbb{T})$ is locally wellposed in $Z_{r}^{s}(\delta), 2<r<4, s \geq 1 / 2$, for some $\delta>0$. This was proved in Theorem 7.2 of [22].

Remark 2.2. Local well-posedness for GDNLS (2.6) implies local existence, uniqueness and continuity of the flow map for DNLS (2.1) [26, 22]. One cannot however carry back to solutions to DNLS all the auxiliary estimates coming from the local well-posedness result for GDNLS.

Now we show how the energy $E(u)$ and $H(u)$ transform under the gauge. Let $u$ be the solution to DNLS (2.1) and define

$$
w=e^{-i J(u)} u .
$$

Then $w$ solves GDNLS (2.6) with the extra $m(w) w_{x}$ term in the linear part of the equation [26]. So the gauge transform is, properly speaking, the transformation $w=e^{-i J(u)} u$ followed by the transformation

$$
v(x, t)=w(t, x-2 m(w) t) .
$$

But all the terms involved in the conserved quantities we considered are invariant under this second transformation $w \mapsto v$ (the torus is invariant under translation). We also notice that $m(u)=m(w)=m(v)$, hence below we will be simply using $m$ for this quantity.

Since

$$
u=e^{i J(w)} w
$$

we have

$$
u_{x}=e^{i J(w)}\left(w_{x}+i J(w)_{x} w\right)
$$

with $J(w)_{x}=|w|^{2}-m$.

We have

$$
\begin{aligned}
H(u) & =\operatorname{Im} \int_{\mathbb{T}} u \overline{u_{x}} d x+\frac{1}{2} \int_{\mathbb{T}}|u|^{4} d x \\
& =\operatorname{Im} \int_{\mathbb{T}} w\left(\overline{w_{x}}-i J(w)_{x} \bar{w}\right) d x+\frac{1}{2} \int_{\mathbb{T}}|w|^{4} d x \\
& =\operatorname{Im} \int_{\mathbb{T}} w \overline{w_{x}} d x-\frac{1}{2} \int_{\mathbb{T}}|w|^{4} d x+2 \pi m^{2}=: \mathscr{H}(w) .
\end{aligned}
$$


In addition

$$
\begin{aligned}
u_{x} \overline{u_{x}} & =\left(w_{x}+i J(w)_{x} w\right)\left(\overline{w_{x}}-i J(w)_{x} \bar{w}\right) \\
& =w_{x} \overline{w_{x}}+i J(w)_{x}\left(w \overline{w_{x}}-\bar{w} w_{x}\right)+J(w)_{x}^{2}|w|^{2} \\
& =w_{x} \overline{w_{x}}-2 \operatorname{Im} J(w)_{x} w \overline{w_{x}}+\left(|w|^{6}-2 m|w|^{4}+m^{2}|w|^{2}\right) \\
& =w_{x} \overline{w_{x}}-2 \operatorname{Im} w^{2} \bar{w} \overline{w_{x}}+2 m \operatorname{Im} w \overline{w_{x}}+\left(|w|^{6}-2 m|w|^{4}+m^{2}|w|^{2}\right) .
\end{aligned}
$$

By the same calculations we also have

$$
u^{2} \overline{u u_{x}}=w^{2} \overline{w w_{x}}-i|w|^{6}+i m|w|^{4} .
$$

We now recall that

$$
E(u)(t)=\int\left|u_{x}\right|^{2} d x+\frac{3}{2} \operatorname{Im} \int u^{2} \bar{u} \overline{u_{x}} d x+\frac{1}{2} \int|u|^{6} d x,
$$

hence by using (2.11), (2.9), (2.10) we find

$E(u)=\int w_{x} \overline{w_{x}} d x-\frac{1}{2} \operatorname{Im} \int w^{2} \bar{w} \overline{w_{x}} d x+2 m \operatorname{Im} \int w \overline{w_{x}} d x-\frac{1}{2} m \int|w|^{4} d x+2 \pi m^{3}$.

If we define

$$
\mathscr{E}(w):=\int_{\mathbb{T}}\left|w_{x}\right|^{2} d x-\frac{1}{2} \operatorname{Im} \int_{\mathbb{T}} w^{2} \bar{w} \overline{w_{x}} d x+\frac{1}{4 \pi}\left(\int_{\mathbb{T}}|w(t)|^{2} d x\right)\left(\int_{\mathbb{T}}|w(t)|^{4} d x\right),
$$

then $E(u)$ can be rewritten as

$$
E(u)=\mathscr{E}(w)+2 m \mathscr{H}(w)-2 \pi m^{3}=: \mathcal{E}(w) .
$$

Remark 2.3. We observe that $H(u)(t)=\mathscr{H}(w)(t)$ and $\frac{d}{d t} H(u)(t)=0$ since $H$ is the Hamiltonian for DNLS (2.1), hence $\frac{d}{d t} \mathscr{H}(w)(t)=0$. On the other hand, we also know that $\frac{d}{d t} E(u)(t)=0$, hence $\frac{d}{d t} \mathscr{E}(w)(t)=0$. By the translation invariance of integration over $\mathbb{T}$, we find that (2.13) holds with $v$ in place of $w$ and

$$
\frac{d}{d t} \mathscr{H}(v)(t)=0=\frac{d}{d t} \mathscr{E}(v)(t) .
$$

\section{Finite-dimensional approximation of GDNLS}

We denote by $P_{N} f=\sum_{|n| \leq N} \widehat{f}(n) e^{i n x}$ the finite-dimensional projection onto the first $2 N+1$ modes and $P_{N}^{\perp}:=I-P_{N}$. Then the finite-dimensional approximation (FGDNLS) is

$$
v_{t}^{N}=i v_{x x}^{N}-P_{N}\left(\left(v^{N}\right)^{2} \overline{v_{x}^{N}}\right)+\frac{i}{2} P_{N}\left(\left|v^{N}\right|^{4} v^{N}\right)-i \psi\left(v^{N}\right)(t) v^{N}-i m\left(v^{N}\right) P_{N}\left(\left|v^{N}\right|^{2} v^{N}\right)
$$

with initial data

$$
v_{0}^{N}=P_{N} v_{0}
$$

where $m$ and $\psi$ are as defined in (2.7) and (2.8) respectively. 
Lemma 3.1. We have

$$
\frac{d}{d t} m\left(v^{N}\right)(t):=\frac{d}{d t} \frac{1}{2 \pi} \int_{\mathbb{T}}\left|v^{N}(x, t)\right|^{2} d x=0 .
$$

Proof. Indeed for simplicity let us momentarily denote by $w:=v^{N}$ a solution to (3.1); note $P_{N} w=w$. Then using that for any $F, \int P_{N}\left(F\left(v^{N}\right)\right) \overline{v^{N}} d x=\int F\left(v^{N}\right) P_{N} \overline{v^{N}} d x=$ $\int F\left(v^{N}\right) \overline{v^{N}} d x$, we obtain

$$
\begin{aligned}
\frac{d}{d t}(2 \pi m(w))= & 2 \operatorname{Re} \int w_{t} \bar{w} d x \\
= & 2 \operatorname{Re}\left(-i \int\left|w_{x}\right|^{2}-\int P_{N}\left(w^{2} \overline{w_{x}}\right) \bar{w}+\frac{i}{2} \int P_{N}\left(|w|^{4} w\right) \bar{w}\right. \\
& \left.-i \psi(w)(t) \int|w|^{2}-i m(w)(t) \int P_{N}\left(|w|^{2} w\right) \bar{w}\right) \\
= & 2 \operatorname{Re}\left(-\int\left(w^{2} \bar{w}_{x}\right) \bar{w}+\frac{i}{2} \int|w|^{6}-i \psi(w) \int|w|^{2}-i m(w) \int|w|^{4}\right) \\
= & -\int w^{2} \bar{w} \bar{w}_{x}-\int w w_{x} \bar{w}^{2}=-\frac{1}{2} \int \partial_{x}\left(|w|^{4}\right)=0 .
\end{aligned}
$$

Theorem 3.2 (Local well-posedness). Let $2<r<4$ and $s \geq 1 / 2$. Then for every

$$
v_{0}^{N} \in B_{R}:=\left\{v_{0}^{N} \in \mathcal{F} L^{s, r}(\mathbb{T}):\left\|v_{0}^{N}\right\| \mathcal{F}_{L^{s, r}(\mathbb{T})}<R\right\}
$$

and $\delta \lesssim R^{-\gamma}$, for some $\gamma>0$, there exists a unique solution

$$
v^{N} \in Z_{r}^{s}(\delta) \subset C\left([-\delta, \delta] ; \mathcal{F} L^{s, r}(\mathbb{T})\right)
$$

of (3.1) and (3.2). Moreover the map

$$
\left(B_{R},\|\cdot\|_{\mathcal{F} L^{s, r}(\mathbb{T})}\right) \rightarrow C\left([-\delta, \delta] ; \mathcal{F} L^{s, r}(\mathbb{T})\right): v_{0}^{N} \mapsto v^{N}
$$

is real analytic.

Proof. The proof follows the argument in [22, Theorem 7.2] since $P_{N}$ acts on a multilinear nonlinearity and it is a bounded operator in $L^{p}, 1<p<\infty$, and commutes with $D^{s}$. Also, although the proof in [22] is presented for $s=1 / 2$, a simple argument of persistence of regularity gives the result for any $s \geq 1 / 2$.

The following lemma gives control on how close the finite-dimensional approximations are to the solution of (2.6). Our proof is a variation of Bourgain's Lemma 2.27 in [3] (see also [12]).

Lemma 3.3 (Approximation lemma). Let $v_{0} \in \mathcal{F} L^{s, r}(\mathbb{T}), s>1 / 2,2<r<4$, be such that $\left\|v_{0}\right\|_{\mathcal{F} L^{s, r}(\mathbb{T})}<A$, for some $A>0$, and let $N$ be a large integer. Assume the solution $v^{N}$ of (3.1) with initial data $v_{0}^{N}(x):=P_{N}\left(v_{0}\right)$ satisfies the bound

$$
\left\|v^{N}(t)\right\|_{\mathcal{F} L^{s, r}(\mathbb{T})} \leq A \quad \text { for all } t \in[-T, T],
$$


for some given $T>0$. Then the IVP GDNLS (2.6) with initial data $v_{0}$ is well-posed on $[-T, T]$ and there exist $C_{0}, C_{1}>0$ such that its solution $v(t)$ satisfies the estimate

$$
\left\|v(t)-v^{N}(t)\right\|_{\mathcal{F} L^{s_{1}, r}(\mathbb{T})} \lesssim \exp \left[C_{0}(1+A)^{C_{1}} T\right] N^{s_{1}-s}
$$

for all $t \in[-T, T]$ and $1 / 2 \leq s_{1}<s$.

Proof. We first observe that from the local well-posedness theory ([22] and Theorem 3.2), GDNLS (2.6) with initial data $v_{0}$ and FGDNLS (3.1) with initial data $v_{0}^{N}$ are both well-posed in $[-\delta, \delta]$ for $\delta \sim(1+A)^{-\gamma}$. Let $w:=v-v^{N}$; then $w$ satisfies the equation

$$
w_{t}-i w_{x x}=F(v)-P_{N} F\left(v^{N}\right)=P_{N}\left[F(v)-F\left(v^{N}\right)\right]+\left(1-P_{N}\right) F(v),
$$

where $F(\cdot)$ is the nonlinearity of (2.6). By the Duhamel principle we have

$w(t)=S(t)\left[v_{0}-v_{0}^{N}\right]+\int_{0}^{t} S\left(t-t^{\prime}\right)\left(P_{N}\left[F(v)-F\left(v^{N}\right)\right]\left(t^{\prime}\right)+\left(1-P_{N}\right) F(v)\left(t^{\prime}\right)\right) d t^{\prime}$,

where $S(t)=e^{i t \Delta}$, and from the proof of Theorem 7.2 in [22] we have the bound

$$
\begin{aligned}
\|w\|_{Z_{r}^{s_{1}}(\delta)} \lesssim & \left\|v_{0}-v_{0}^{N}\right\| \mathcal{F} L^{s_{1}, r}(\mathbb{T}) \\
& +\|\left(1-\delta^{\gamma}\left(1+\left\|v^{N}\right\|_{Z_{r}^{s_{1}}(\delta)}+\|v\|_{Z_{r}^{s_{1}}(\delta)}\right)^{4}\|w\|_{Z_{r}^{s_{1}}(\delta)} S\left(t-t^{\prime}\right) F(v)\left(t^{\prime}\right) d t^{\prime} \|_{Z_{r}^{s_{1}}(\delta)}\right. \\
\lesssim & A N^{s_{1}-s}+\delta^{\gamma}\left(1+\left\|v^{N}\right\|_{Z_{r}^{s_{1}}(\delta)}+\|v\|_{Z_{r}^{s_{1}}(\delta)}\right)^{4}\|w\|_{Z_{r}^{s_{1}}(\delta)} \\
& +N^{s_{1}-s} \delta^{\gamma}\left(1+\|v\|_{Z_{r}^{s}(\delta)}\right)^{5}
\end{aligned}
$$

By choosing a smaller $\delta$ if necessary we obtain from (3.4)

$$
\|w\|_{Z_{r}^{s_{1}}(\delta)} \leq C A N^{s_{1}-s}+\frac{1}{2}\|w\|_{Z_{r}^{s_{1}}(\delta)}
$$

for some absolute constant $C>0$,which implies

$$
\left\|v(t)-v^{N}(t)\right\|_{\mathcal{F} L^{s_{1}, r}(\mathbb{T})} \leq 2 C A N^{s_{1}-s} \quad \text { for all } t \in[-\delta, \delta]
$$

and by iteration (3.3) follows.

\section{Analysis of the finite-dimensional equation FGDNLS}

Recall that equation DNLS is Hamiltonian and hence its gauge equivalent formulation should stay Hamiltonian (change of coordinates). However, the gauge transformation is not a 'canonical map' and the symplectic form in the new coordinates depends on $v$; that is, we lose the simple expression the symplectic form (namely $\partial_{x}$ ) had in the original coordinates. Two problems arise from the lack of commutativity between the gauged skew-selfadjoint form $J$ and $P_{N}$ : 
(1) The conservation of Lebesgue measure associated to FGDNLS is not obvious as before. We must prove that this is indeed the case; see Subsection 4.1 below.

And more seriously:

(2) We lose the conservation of the energy $\mathcal{E}\left(v^{N}\right)$ for the finite-dimensional approximations, that is, $d \mathcal{E}\left(v^{N}\right) / d t \neq 0$. In particular we lose the invariance of $\mu_{N}$, the associated finite-dimensional weighted Wiener measure. However we have an estimate controlling its growth, namely Theorem 4.2 below.

\subsection{Invariance of the Lebesgue measure}

If we rewrite FGDNLS (3.1) as a system of complex ODE's for the Fourier coefficients $c_{k} \equiv \widehat{v^{N}}(k)$ we obtain a set of $2 N+1$ complex equations of the form $\frac{d}{d t} c_{k}=F_{k}\left(\left\{c_{j}, \bar{c}_{j}\right\}\right)$, or equivalently $4 N+2$ equations $\frac{d}{d t} a_{k}=\operatorname{Re} F_{k}\left(\left\{c_{j}, \bar{c}_{j}\right\}\right)$ and $\frac{d}{d t} b_{k}=\operatorname{Im} F_{k}\left(\left\{c_{j}, \bar{c}_{j}\right\}\right)$ for the real functions $a_{k}=\operatorname{Re} F_{k}$ and $b_{k}=\operatorname{Im} F_{k}$.

To show that this set of equations preserves volume we need to verify that the divergence of the vector field vanishes, i.e.,

$$
\sum_{k}\left(\frac{\partial \operatorname{Re} F_{k}}{\partial a_{k}}+\frac{\partial \operatorname{Im} F_{k}}{\partial b_{k}}\right)=0
$$

This is easily shown to be equivalent to

$$
\sum_{k}\left(\frac{\partial F_{k}}{\partial c_{k}}+\frac{\partial \bar{F}_{k}}{\partial \bar{c}_{k}}\right)=0
$$

And indeed we have

Lemma 4.1. The Lebesgue measure $\prod_{|j| \leq N} d a_{j} d b_{j}$ is invariant under the flow of the system of ODE's (4.1).

Proof. The FGDNLS (3.1) as a system of complex ODE's for the Fourier coefficients $c_{k}$ takes the form

$$
\begin{aligned}
\frac{d}{d t} c_{k}= & -i k^{2} c_{k}+i \sum_{n_{1}, n_{2}, n_{3}} n_{3} c_{n_{1}} c_{n_{2}} \bar{c}_{n_{3}} \delta_{n_{1}+n_{2}-n_{3}-k} \\
& +\frac{i}{2} \sum_{n_{1}, n_{2}, n_{3}, n_{4}, n_{5}} c_{n_{1}} c_{n_{2}} c_{n_{3}} \bar{c}_{n_{4}} \bar{c}_{n_{5}} \delta_{n_{1}+n_{2}+n_{3}-n_{4}-n_{5}-k} \\
& -i \psi\left(\left\{c_{j}, \bar{c}_{j}\right\}\right) c_{k}-i m\left(\left\{c_{j}, \bar{c}_{j}\right\}\right) \sum_{n_{1}, n_{2}, n_{3}} c_{n_{1}} c_{n_{2}} \bar{c}_{n_{3}} \delta_{n_{1}+n_{2}-n_{3}-k}
\end{aligned}
$$

with $m\left(\left\{c_{j}, \bar{c}_{j}\right\}\right)=\sum_{j}\left|c_{j}\right|^{2}$ and

$$
\psi\left(\left\{c_{j}, \bar{c}_{j}\right\}\right)=-2 \sum_{k} k\left|c_{k}\right|^{2}+\frac{1}{2} \sum_{n_{1}, n_{2}, n_{3}, n_{4}} c_{n_{1}} c_{n_{2}} \bar{c}_{n_{3}} \bar{c}_{n_{4}} \delta_{n_{1}+n_{2}-n_{3}-n_{4}}-\left(\sum_{j}\left|c_{j}\right|^{2}\right)^{2} .
$$


To show that this set of equations preserves volume we need to verify

$$
\sum_{k}\left(\frac{\partial F_{k}}{\partial c_{k}}+\frac{\partial \bar{F}_{k}}{\partial \bar{c}_{k}}\right)=0
$$

The vector field $F_{k}$ consists of several terms which we analyze separately.

(1) $F_{k}^{(1)}=-i k^{2} c_{k}$. Then $\frac{\partial F_{k}^{(1)}}{\partial c_{k}}+\frac{\partial \bar{F}_{k}^{(1)}}{\partial \bar{c}_{k}}=-i k^{2}+i k^{2}=0$.

(2) $F_{k}^{(2)}=i \sum_{n_{1}, n_{2}, n_{3}} n_{3} c_{n_{1}} c_{n_{2}} \bar{c}_{n_{3}} \delta_{n_{1}+n_{2}-n_{3}-k}$. To differentiate we consider the terms with $n_{1}=k$ and $n_{2}=k$ to obtain

$$
\frac{\partial F_{k}^{(2)}}{\partial c_{k}}=i 2 \pi \sum_{n_{2}, n_{3}} n_{3} c_{n_{2}} \bar{c}_{n_{3}} \delta_{n_{2}-n_{3}}+i 2 \pi \sum_{n_{1}, n_{3}} n_{3} c_{n_{1}} \bar{c}_{n_{3}} \delta_{n_{1}-n_{3}}=i 4 \pi \sum_{n} n\left|c_{n}\right|^{2}
$$

and similarly

$$
\frac{\partial \bar{F}_{k}^{(2)}}{\partial \bar{c}_{k}}=-i 4 \pi \sum_{n} n\left|c_{n}\right|^{2},
$$

and thus all the contributions of this term to the divergence disappear.

(3) $F_{k}^{(3)}=\frac{i}{2} \sum_{n_{1}, n_{2}, n_{3}, n_{4}, n_{5}} c_{n_{1}} c_{n_{2}} c_{n_{3}} \bar{c}_{n_{4}} \bar{c}_{n_{5}} \delta_{n_{1}+n_{2}+n_{3}-n_{4}-n_{5}-k}$. This term is treated similarly to (2) and is left to the reader.

(4) $F_{k}^{(4)}=2 i\left(\sum_{j} j\left|c_{j}\right|^{2}\right) c_{k}$. We have

$$
\frac{\partial F_{k}^{(4)}}{\partial c_{k}}=2 i k\left|c_{k}\right|^{2}+2 i \sum_{j} j\left|c_{j}\right|^{2}, \quad \frac{\partial \bar{F}_{k}^{(4)}}{\partial \bar{c}_{k}}=-2 i k\left|c_{k}\right|^{2}-2 i \sum_{j} j\left|c_{j}\right|^{2},
$$

and so these terms do not contribute to the divergence.

(5) $F_{k}^{(5)}=i\left(\sum_{j}\left|c_{j}\right|^{2}\right)^{2} c_{k}$. We have

$$
\frac{\partial F_{k}^{(5)}}{\partial c_{k}}=2 i\left(\sum_{j}\left|c_{j}\right|^{2}\right)\left|c_{k}\right|^{2}+i\left(\sum_{j}\left|c_{j}\right|^{2}\right)^{2}
$$

and again $\frac{\partial F_{k}^{(5)}}{\partial c_{k}}+\frac{\partial \bar{F}_{k}^{(5)}}{\partial \bar{c}_{k}}=0$

(6) $F_{k}^{(6)}=-\frac{i}{2} \sum_{n_{1}, n_{2}, n_{3}, n_{4}} c_{n_{1}} c_{n_{2}} \bar{c}_{n_{3}} \bar{c}_{n_{4}} \delta_{n_{1}+n_{2}-n_{3}-n_{4}} c_{k}$. We have

$$
\begin{aligned}
\frac{\partial F_{k}^{(6)}}{\partial c_{k}}= & -\frac{i}{2} \sum_{n_{1}, n_{2}, n_{3}, n_{4}} c_{n_{1}} c_{n_{2}} \bar{c}_{n_{3}} \bar{c}_{n_{4}} \delta_{n_{1}+n_{2}-n_{3}-n_{4}} \\
& -i \sum_{n_{2}, n_{3}, n_{4}} c_{k} c_{n_{2}} \bar{c}_{n_{3}} \bar{c}_{n_{4}} \delta_{k+n_{2}-n_{3}-n_{4}}
\end{aligned}
$$


and

$$
\begin{aligned}
\frac{\partial \bar{F}_{k}^{(6)}}{\partial \bar{c}_{k}}= & +\frac{i}{2} \sum_{n_{1}, n_{2}, n_{3}, n_{4}} \bar{c}_{n_{1}} \bar{c}_{n_{2}} c_{n_{3}} c_{n_{4}} \delta_{n_{1}+n_{2}-n_{3}-n_{4}} \\
& +i \sum_{n_{2}, n_{3}, n_{4}} \bar{c}_{k} \bar{c}_{n_{2}} c_{n_{3}} c_{n_{4}} \delta_{k+n_{2}-n_{3}-n_{4} .} .
\end{aligned}
$$

The first terms in (4.2) and (4.3) cancel for each $k$. By summing the second terms over $k$, we see that they do not contribute to the divergence.

(7) $F_{k}^{(7)}=-i \sum_{j}\left|c_{j}\right|^{2} \sum_{n_{1}, n_{2}, n_{3}} c_{n_{1}} c_{n_{2}} \bar{c}_{n_{3}} \delta_{n_{1}+n_{2}-n_{3}-k}$. We have

$$
\begin{aligned}
& \frac{\partial F_{k}^{(7)}}{\partial c_{k}}=-i \sum_{n_{1}, n_{2}, n_{3}} c_{n_{1}} c_{n_{2}} \bar{c}_{n_{3}} \bar{c}_{k} \delta_{n_{1}+n_{2}-n_{3}-k}-2 i\left(\sum_{j}\left|c_{j}\right|^{2}\right)^{2}, \\
& \frac{\partial \bar{F}_{k}^{(7)}}{\partial \bar{c}_{k}}=i \sum_{n_{1}, n_{2}, n_{3}} \bar{c}_{n_{1}} \bar{c}_{n_{2}} c_{n_{3}} c_{k} \delta_{n_{1}+n_{2}-n_{3}-k}+2 i\left(\sum_{j}\left|c_{j}\right|^{2}\right)^{2} .
\end{aligned}
$$

The second terms add to 0 for each $k$ while the first terms cancel if we sum over all $k$.

\subsection{Energy growth estimate}

Theorem 4.2. Let $v^{N}(t)$ be a solution to FGDNLS (3.1) in $[-\delta, \delta]$, and let $K>0$ be such that $\left\|v^{N}\right\|_{X_{3}^{(2 / 3)-, 1 / 2}(\delta)} \leq K$. Then there exists $\beta>0$ such that

$$
\left|\mathcal{E}\left(v^{N}(\delta)\right)-\mathcal{E}\left(v^{N}(0)\right)\right|=\left|\int_{0}^{\delta} \frac{d}{d t} \mathcal{E}\left(v^{N}\right)(t) d t\right| \lesssim C(\delta) N^{-\beta} \max \left(K^{6}, K^{8}\right) .
$$

Remark 4.3. It is possible that the estimate (4.4) may still hold for a different choice of $X_{r}^{s, 1 / 2}(\delta)$ norm, with $s \geq 1 / 2,2<r<4$ so that local well-posedness holds. On the other hand the pair $(s, r)$ should also be such that $(s-1) \cdot r<-1$ in order for $\mathcal{F} L^{s, r}$ to contain the support of the Wiener measure (cf. Section 5). Our choice of $s=(2 / 3)-$ and $r=3$ allows us to prove (4.4) while satisfying the conditions for local well-posedness and the support of the measure. Note that $\mathcal{F} L^{(2 / 3)-, 3}$ scales like $H^{(1 / 2)-}$.

\subsection{Preparation for the proof of Theorem 4.2}

Let $v^{N}$ denote the solution of FGDNLS (3.1) which we rewrite as

$$
v_{t}^{N}=\mathcal{L} v^{N}+P_{N}^{\perp}\left(\left(v^{N}\right)^{2} \overline{v_{x}^{N}}\right)-\frac{i}{2} P_{N}^{\perp}\left(\left|v^{N}\right|^{4} v^{N}\right)+i m\left(v^{N}\right) P_{N}^{\perp}\left(\left|v^{N}\right|^{2} v^{N}\right),
$$

where

$$
\mathcal{L} v^{N}:=i v_{x x}^{N}-\left(v^{N}\right)^{2} \overline{v_{x}^{N}}+\frac{i}{2}\left|v^{N}\right|^{4} v^{N}-i \psi\left(v^{N}\right) v^{N}-i m\left(v^{N}\right)\left|v^{N}\right|^{2} v^{N} .
$$


We first observe that from (2.13) and Lemma 3.1 we have

$$
\frac{d}{d t} \mathcal{E}\left(v^{N}\right)=\frac{d}{d t} \mathscr{E}\left(v^{N}\right)+2 m_{N} \frac{d}{d t} \mathscr{H}\left(v^{N}\right),
$$

where $m_{N}:=m\left(v^{N}\right)$.

Lemma 4.4. With the above notation we have

$$
\begin{aligned}
& \frac{d}{d t} \mathscr{E}\left(v^{N}\right)(t)=-2 \operatorname{Im} \int v^{N} \overline{v^{N} v_{x}^{N}} P_{N}^{\perp}\left(\left(v^{N}\right)^{2} \overline{v_{x}^{N}}\right) d x+\operatorname{Re} \int v^{N} \overline{v^{N} v_{x}^{N}} P_{N}^{\perp}\left(\left|v^{N}\right|^{4} v^{N}\right) d x \\
& -2 m_{N} \operatorname{Re} \int v^{N} \overline{v^{N} v_{x}^{N}} P_{N}^{\perp}\left(\left|v^{N}\right|^{2} v^{N}\right) d x+2 m_{N} \operatorname{Re} \int v^{N}{\overline{v^{N}}}^{2} P_{N}^{\perp}\left(\left(v^{N}\right)^{2} \overline{v_{x}^{N}}\right) d x \\
& +m_{N} \operatorname{Im} \int v^{N}{\overline{v^{N}}}^{2} P_{N}^{\perp}\left(\left|v^{N}\right|^{4} v^{N}\right) d x-2 m_{N}^{2} \operatorname{Im} \int v^{N}{\overline{v^{N}}}^{2} P_{N}^{\perp}\left(\left|v^{N}\right|^{2} v^{N}\right) d x, \\
& \frac{d}{d t} \mathscr{H}\left(v^{N}\right)(t)=-2 \operatorname{Re} \int_{\mathbb{T}}\left(\overline{v^{N}}\right)^{2} v^{N} P_{N}^{\perp}\left(\left(v^{N}\right)^{2} \overline{v_{x}^{N}}\right) d x \\
& +\operatorname{Im} \int v^{N}\left(\overline{v^{N}}\right)^{2} P_{N}^{\perp}\left(\left|v^{N}\right|^{4} v^{N}\right) d x-2 m_{N} \operatorname{Im} \int v^{N}\left(\overline{v^{N}}\right)^{2} P_{N}^{\perp}\left(\left|v^{N}\right|^{2} v^{N}\right) d x,
\end{aligned}
$$

and

$\frac{d}{d t} \mathcal{E}\left(v^{N}\right)(t)=-2 \operatorname{Im} \int v^{N} \overline{v^{N} v_{x}^{N}} P_{N}^{\perp}\left(\left(v^{N}\right)^{2} \overline{v_{x}^{N}}\right) d x+\operatorname{Re} \int v^{N} \overline{v^{N} v_{x}^{N}} P_{N}^{\perp}\left(\left|v^{N}\right|^{4} v^{N}\right) d x$

$-2 m_{N} \operatorname{Re} \int v^{N} \overline{v^{N} v_{x}^{N}} P_{N}^{\perp}\left(\left|v^{N}\right|^{2} v^{N}\right) d x-2 m_{N} \operatorname{Re} \int_{\mathbb{T}}\left(\overline{v^{N}}\right)^{2} v^{N} P_{N}^{\perp}\left(\left(v^{N}\right)^{2} \overline{v_{x}^{N}}\right) d x$

$+3 m_{N} \operatorname{Im} \int v^{N}\left(\overline{v^{N}}\right)^{2} P_{N}^{\perp}\left(\left|v^{N}\right|^{4} v^{N}\right) d x-6 m_{N} \operatorname{Im} \int v^{N}\left(\overline{v^{N}}\right)^{2} P_{N}^{\perp}\left(\left|v^{N}\right|^{2} v^{N}\right) d x$.

Proof. From (2.12) and integration by parts we have

$$
\begin{aligned}
\frac{d}{d t} \mathscr{E}\left(v^{N}\right)(t)= & -2 \operatorname{Re} \int v_{t}^{N} \overline{v_{x x}^{N}} d x-2 \operatorname{Im} \int v^{N} v_{t}^{N} \overline{v^{N} v_{x}^{N}} d x \\
& +2 m_{N} \operatorname{Re} \int v^{N} v_{t}^{N}{\overline{v^{N}}}^{2} d x
\end{aligned}
$$

Due to the energy conservation for the GDNLS (infinite system), one can see that the contribution in (4.10) from $\mathcal{L} v^{N}$ defined in (4.5) is zero. On the other hand by orthogonality we also have

$$
-2 \operatorname{Re} \int \overline{v_{x x}^{N}}\left(P_{N}^{\perp}\left(\left(v^{N}\right)^{2} \overline{v_{x}^{N}}\right)-\frac{i}{2} P_{N}^{\perp}\left(\left|v^{N}\right|^{4} v^{N}\right)+i m\left(v^{N}\right) P_{N}^{\perp}\left(\left|v^{N}\right|^{2} v^{N}\right)\right) d x=0 .
$$

Hence (4.7) follows. By a similar argument we obtain (4.8) as well. The lemma follows by substituting (4.7) and (4.8) into (4.6).

Remark 4.5. To establish Theorem 4.2 we need to estimate the terms in (4.9). In doing so we will ignore absolute constants and whether we are looking at the real or imaginary parts of the terms. 
The first term in (4.9) gives a contribution to (4.4) which is essentially

$$
I_{1}=\int_{0}^{\delta} \int v^{N} \overline{v^{N} v_{x}^{N}} P_{N}^{\perp}\left(\left(v^{N}\right)^{2} \overline{v_{x}^{N}}\right) d x d t .
$$

This term is the hardest to control since it has two derivatives, so we will treat this one first. We start by discussing how to absorb the rough time cut-off. Assume $\phi$ is any function in $X_{3}^{(2 / 3)-, 1 / 2}$ such that

Then we write

$$
\left.\phi\right|_{[-\delta, \delta]}=v^{N} .
$$

$$
\begin{aligned}
I_{1} & =\int_{\mathbb{T} \times \mathbb{R}} \chi_{[0, \delta]}(t) P_{N}^{\perp}\left(\left(v^{N}\right)^{2} \partial_{x} \overline{v^{N}}\right) v^{N} \overline{v^{N} v_{x}^{N}} d x d t \\
& =\int_{\mathbb{T} \times \mathbb{R}} P_{N}^{\perp}\left(\left(\chi_{[0, \delta]} \phi^{N}\right)^{2} \chi_{[0, \delta]} \overline{\phi_{x}^{N}}\right) \chi_{[0, \delta]} \phi^{N} \chi_{[0, \delta]} \overline{\phi^{N}} \chi_{[0, \delta]} \overline{\phi_{x}^{N}} d x d t
\end{aligned}
$$

and by denoting

we will in fact show that

$$
w:=\chi_{[0, \delta]} \phi, \quad w=P_{N}(w),
$$

$$
\left|I_{1}\right|=\left|\int_{\mathbb{T} \times \mathbb{R}} P_{N}^{\perp}\left((w)^{2} \partial_{x} \bar{w}\right) w \overline{w w_{x}} d x d t\right| \leq C(\delta) N^{-\beta}\|w\|_{X_{3}^{\frac{2}{3}-, \frac{1}{2}-}}^{6} .
$$

To go back to $v^{N}$ we use the following lemma:

Lemma 4.6 (time cutoff). Let $b<b_{1}<1 / 2$. Then the exists $C^{\prime}(\delta)>0$ such that

$$
\|w\|_{X_{3}^{\frac{2}{3}-, b}} \leq C^{\prime}(\delta)\|\phi\|_{X_{3}^{\frac{2}{3}-, b_{1}}} \leq C^{\prime}(\delta)\left\|v^{N}\right\|_{X_{3}^{\frac{2}{3}-, \frac{1}{2}}(\delta)}
$$

where $w, \phi$ and $v^{N}$ are as above.

Proof. Since the regularity in $x$ does not play any role, without any loss of generality we ignore the power $s=(2 / 3)-$. Then

$$
\begin{aligned}
\|w\|_{X_{3}^{0, b}} & =\left(\sum_{n}\left(\int\left|\widehat{\chi_{[0, \delta]} \phi}(n, \tau)\right|^{2}\left\langle\tau+n^{2}\right\rangle^{2 b} d \tau\right)^{3 / 2}\right)^{1 / 3} \\
& =\left(\sum_{n}\left(\int\left|\int_{\tau_{1}} \widehat{\chi_{[0, \delta]}}\left(\tau-\tau_{1}\right) \widehat{\phi}\left(n, \tau_{1}\right) d \tau_{1}\right|^{2}\left\langle\tau+n^{2}\right\rangle^{2 b} d \tau\right)^{3 / 2}\right)^{1 / 3} .
\end{aligned}
$$

Writing $\tau+n^{2}=\left(\tau-\tau_{1}\right)+\left(\tau_{1}+n^{2}\right)$ we bound (4.12) by

$$
\begin{aligned}
\lesssim & \left(\sum_{n}\left(\int\left|\int_{\tau_{1}} \widehat{\chi_{[0, \delta]}}\left(\tau-\tau_{1}\right)\left\langle\tau-\tau_{1}\right\rangle^{b} \widehat{\phi}\left(n, \tau_{1}\right) d \tau_{1}\right|^{2} d \tau\right)^{3 / 2}\right)^{1 / 3} \\
& +\left(\sum_{n}\left(\int\left|\int_{\tau_{1}} \widehat{\chi_{[0, \delta]}}\left(\tau-\tau_{1}\right) \widehat{\phi}\left(n, \tau_{1}\right)\left\langle\tau_{1}+n^{2}\right\rangle^{b} d \tau_{1}\right|^{2} d \tau\right)^{3 / 2}\right)^{1 / 3}
\end{aligned}
$$

We treat the first sum (4.13), the second one (4.14) being similar. If $\left\langle\tau-\tau_{1}\right\rangle<\left\langle\tau_{1}+n^{2}\right\rangle$ 
then by Young's inequality, (4.13) can be bounded by

$$
\lesssim\left\|\widehat{\chi[0, \delta]}(\tau) /\langle\tau\rangle^{\varepsilon}\right\|_{L^{1}}\|\| \widehat{\phi}(\tau, n)\left\langle\tau+n^{2}\right\rangle^{b+\varepsilon}\left\|_{L^{2}}\right\|_{\ell^{3}} \lesssim\|\chi\|_{H^{\beta}}\|\phi\|_{X_{3}^{0, b_{1}}}
$$

by Cauchy-Schwarz on the $\widehat{\chi}$ term provided $\beta+\varepsilon>1 / 2, \beta<1 / 2$ and where $b_{1}:=$ $b+\varepsilon<1 / 2$.

On the other hand if $\left\langle\tau-\tau_{1}\right\rangle \geq\left\langle\tau_{1}+n^{2}\right\rangle$, then again by Young's inequality, (4.13) can be bounded by

$$
\lesssim\left\|\widehat{\chi[0, \delta]}(\tau)\langle\tau\rangle^{b+\varepsilon}\right\|_{L^{2}}\|\| \widehat{\phi}(\tau, n)\left\langle\tau+n^{2}\right\rangle^{-\varepsilon}\left\|_{L^{1}}\right\|_{\ell^{3}} \lesssim\|\chi\|_{H^{b+\varepsilon}}\|\phi\|_{X_{3}^{0, b_{1}}}
$$

by Cauchy-Schwarz on the $\widehat{\phi}$ term provided $b_{1}+\varepsilon>1 / 2$ and $b_{1}<1 / 2$. Finally by taking the infimum and using the definition of $X_{3}^{0,1 / 2}(\delta)$, a bound in terms of $\left\|v^{N}\right\|_{X_{3}^{0,1 / 2}(\delta)}$ follows.

\subsection{Proof of Theorem 4.2}

Returning to (4.11) we write

$$
\begin{aligned}
& I_{1}=\int_{\mathbb{T} \times \mathbb{R}} P_{N}^{\perp}\left(w^{2} \partial_{x} \bar{w}\right) w \overline{w w_{x}} d x d t \\
& =\int_{\tau} \sum_{|n|>N}\left(\widehat{w^{2} \overline{w_{x}}}\right)(n, \tau) \overline{\left(\widehat{\bar{w} w_{x}}\right)}(n, \tau) d \tau \\
& =\int \sum_{|n|>N}\left(\int_{\tau=\tau_{1}+\tau_{2}-\tau_{3}} \sum_{\substack{n=n_{1}+n_{2}-n_{3} \\
\left|n_{j}\right| \leq N}} \widehat{w}\left(n_{1}, \tau_{1}\right) \widehat{w}\left(n_{2}, \tau_{2}\right)\left(-i n_{3}\right) \widehat{w}\left(n_{3}, \tau_{3}\right) d \tau_{1} d \tau_{2}\right) \\
& \times\left(\int_{-\tau=\tau_{4}-\tau_{5}-\tau_{6}} \sum_{\substack{n=n_{4}-n_{5}-n_{6} \\
\left|n_{j}\right| \leq N}} \widehat{w}\left(n_{4}, \tau_{4}\right) \widehat{\widehat{w}}\left(n_{5}, \tau_{5}\right)\left(-i n_{6}\right) \widehat{\widehat{w}}\left(n_{6}, \tau_{6}\right) d \tau_{4} d \tau_{5}\right) d \tau \\
& =\int \sum_{N<|n| \leq 3 N}\left(\int_{\tau=\tau_{1}+\tau_{2}+\tau_{3}} \sum_{\substack{n=n_{1}+n_{2}+n_{3} \\
\left|n_{j}\right| \leq N}} \widehat{w}\left(n_{1}, \tau_{1}\right) \widehat{w}\left(n_{2}, \tau_{2}\right)\left(i n_{3}\right) \widehat{\bar{w}}\left(n_{3}, \tau_{3}\right) d \tau_{1} d \tau_{2}\right) \\
& \times\left(\int_{-\tau=\tau_{4}+\tau_{5}+\tau_{6}} \sum_{\substack{n=n_{4}+n_{5}+n_{6} \\
\left|n_{j}\right| \leq N}} \widehat{w}\left(n_{4}, \tau_{4}\right) \widehat{\bar{w}}\left(n_{5}, \tau_{5}\right)\left(i n_{6}\right) \widehat{\bar{w}}\left(n_{6}, \tau_{6}\right) d \tau_{4} d \tau_{5}\right) d \tau \\
& =\int \sum_{N<|n| \leq 3 N}\left(\int_{\tau=\tau_{1}+\tau_{2}+\tau_{3}} \sum_{\substack{n=n_{1}+n_{2}+n_{3} \\
\left|n_{j}\right| \leq N}} \widehat{w_{1}}\left(n_{1}, \tau_{1}\right) \widehat{w_{2}}\left(n_{2}, \tau_{2}\right)\left(i n_{3}\right) \widehat{w_{3}}\left(n_{3}, \tau_{3}\right) d \tau_{1} d \tau_{2}\right) \\
& \times\left(\int_{-\tau=\tau_{4}+\tau_{5}+\tau_{6}} \sum_{\substack{n=n_{4}+n_{5}+n_{6} \\
\left|n_{j}\right| \leq N}} \widehat{w_{4}}\left(n_{4}, \tau_{4}\right) \widehat{w_{5}}\left(n_{5}, \tau_{5}\right)\left(i n_{6}\right) \widehat{w_{6}}\left(n_{6}, \tau_{6}\right) d \tau_{4} d \tau_{5}\right) d \tau,
\end{aligned}
$$

where $w_{1}=w_{2}=w_{4}=w$ and $\overline{w_{3}}=\overline{w_{5}}=\overline{w_{6}}=\bar{w}$. 
Remark 4.7. In what follows we always think of $N_{j}, N$ as dyadic; more precisely $N_{j}$ $:=2^{K_{j}}, N:=2^{K}$ where $K_{j}<K$ since $n_{j} \in \mathbb{Z}$. By a slight abuse of notation we then denote by $N_{j}$ both $\left|n_{j}\right|$ and the dyadic interval $\left[2^{K_{j}}, 2^{K_{j}+1}\right.$ ) to which $\left|n_{j}\right|$ belongs when $n_{j} \neq 0$. Moreover we denote by $w_{N_{j}}$ the function such that $\widehat{w_{N_{j}}}\left(n_{j}\right)=\chi_{\left\{\left|n_{j}\right| \sim N_{j}\right\}} \widehat{w_{j}}\left(n_{j}\right)$.

From the expression above we then have

$$
\begin{aligned}
& \left|n_{j}\right| \leq N, \quad N \leq|n| \leq 3 N, \quad n=n_{1}+n_{2}+n_{3}, \quad-n=n_{4}+n_{5}+n_{6}, \\
& N \sim \max \left(N_{1}, N_{2}, N_{3}\right) \sim \max \left(N_{4}, N_{5}, N_{6}\right), \\
& \tau+n^{2}-\left(\tau_{1}+n_{1}^{2}\right)-\left(\tau_{2}+n_{2}^{2}\right)-\left(\tau_{3}-n_{3}^{2}\right)=2\left(n-n_{1}\right)\left(n-n_{2}\right), \\
& \tau+n^{2}+\left(\tau_{4}+n_{4}^{2}\right)+\left(\tau_{5}-n_{5}^{2}\right)+\left(\tau_{6}-n_{6}^{2}\right)=2\left(n+n_{5}\right)\left(n+n_{6}\right) .
\end{aligned}
$$

So if we let $\tilde{\sigma}_{j}:=\tau_{j} \pm n_{j}^{2}$ and $\sigma_{j}:=\left\langle\tau_{j} \pm n_{j}^{2}\right\rangle$, by subtracting (4.17) from (4.18) we have

$$
\sum_{j=1}^{6} \tilde{\sigma}_{j}=-2\left(n\left(n_{1}+n_{2}+n_{5}+n_{6}\right)-n_{1} n_{2}+n_{5} n_{6}\right) .
$$

This in turn can also be rewritten using $n_{1}+n_{2}+n_{3}+n_{4}+n_{5}+n_{6}=0$ or $n=n_{1}+n_{2}+n_{3}$ and $-n=n_{4}+n_{5}+n_{6}$ as

$$
\sum_{j=1}^{6} \tilde{\sigma}_{j}=2\left(n\left(n_{3}+n_{4}\right)+n_{1} n_{2}-n_{5} n_{6}\right) .
$$

In addition, since $\tau_{1}+\tau_{2}+\tau_{3}+\tau_{4}+\tau_{5}+\tau_{6}=0$, adding and subtracting $n_{j}^{2}, j=1, \ldots, 6$, in the appropriate fashion, we obtain

$$
\sum_{j=1}^{6} \tilde{\sigma}_{j}=\left(n_{3}^{2}+n_{5}^{2}+n_{6}^{2}\right)-\left(n_{1}^{2}+n_{2}^{2}+n_{4}^{2}\right) .
$$

Hence we need to estimate

$$
\begin{aligned}
& \left|I_{1}\right|=\left|\sum_{N_{i} \leq N ; i=1, \ldots 6} \int_{\mathbb{R}} \int_{\mathbb{T}} P_{N}^{\perp}\left(w_{N_{1}} w_{N_{2}} \partial_{x} \overline{w_{N_{3}}}\right) w_{N_{4}} \overline{w_{N_{5}}} \partial_{x} \overline{w_{N_{6}}} d x d t\right| \\
& =\mid \sum_{N_{i} \leq N ; i=1, \ldots 6} \sum_{|n| \geq N} \int_{\tau}\left(\int_{\tau=\tau_{1}+\tau_{2}+\tau_{3}} \sum_{n=n_{1}+n_{2}+n_{3}} \widehat{w_{N_{1}}} \widehat{w_{N_{2}}}\left(i n_{3}\right) \widehat{\widehat{w_{N_{3}}}} d \tau_{1} d \tau_{2}\right) \\
& \times\left(\int_{-\tau=\tau_{4}+\tau_{5}+\tau_{6}} \sum_{-n=n_{4}+n_{5}+n_{6}} \widehat{w_{N_{4}}} \widehat{w_{N_{5}}}\left(i n_{6}\right) \widehat{\bar{w}_{N_{6}}} d \tau_{4} d \tau_{5}\right) d \tau \mid \\
& \leq \sum_{N \leq|n| \leq 3 N} \sum_{N_{i} \leq N ; i=1, \ldots 6} \int_{\tau}\left(\int_{\tau=\tau_{1}+\tau_{2}+\tau_{3}} \sum_{n=n_{1}+n_{2}+n_{3}}\left|\widehat{w_{N_{1}}}\right|\left|\widehat{w_{N_{2}}}\right|\left|n_{3}\right|\left|\widehat{w_{N_{3}}}\right| d \tau_{1} d \tau_{2}\right) \\
& \times\left(\int_{-\tau=\tau_{4}+\tau_{5}+\tau_{6}} \sum_{-n=n_{4}+n_{5}+n_{6}}\left|\widehat{w_{N_{4}}}\right|\left|\widehat{w_{N_{5}}}\right|\left|n_{6}\right|\left|\widehat{w_{N_{6}}}\right| d \tau_{4} d \tau_{5}\right) d \tau \text {. }
\end{aligned}
$$


Remark 4.8. This expression (4.22) will be our point of departure in beginning our estimate. In what follows we will abuse notation and write $w_{N_{j}}$ for $\left|\widehat{w_{N_{j}}}\right|$ and $\overline{w_{N_{k}}}$ for $\left|\widehat{\overline{w_{N_{k}}}}\right|$ since at the end we will estimate all functions in the $X_{r}^{s, b}$ norms which depend solely on the absolute value of the Fourier transform.

We start by laying out all possible cases and organizing them according to the sizes of the two derivative terms.

\section{Types:}

I. $N_{3} \sim N$ and $N_{6} \sim N$,

II. $N_{3} \sim N$ and $N_{6} \ll N$,

III. $N_{6} \sim N$ and $N_{3} \ll N$,

IV. $N_{3} \ll N$ and $N_{6} \ll N$.

Now we subdivide into all subcases in each situation and group them according to how many low frequencies (i.e. $N_{j} \ll N$ ) we have overall, taking into account (4.16).

\section{All cases for each type:}

IA. $N_{3} \sim N, N_{6} \sim N$ and 4 lows: $N_{1}, N_{2}, N_{4}, N_{5} \ll N$.

IB. $N_{3} \sim N, N_{6} \sim N$ and 3 lows:

(i) $N_{1}, N_{2}, N_{4} \ll N$ and $N_{5} \sim N$,

(ii) $N_{1}, N_{2}, N_{5} \ll N$ and $N_{4} \sim N$,

(iii) $N_{1}, N_{4}, N_{5} \ll N$ and $N_{2} \sim N$,

(iv) $N_{2}, N_{4}, N_{5} \ll N$ and $N_{1} \sim N$.

IC. $N_{3} \sim N, N_{6} \sim N$ and 2 lows:

(i) $N_{1}, N_{2} \ll N$ and $N_{4}, N_{5} \sim N$,

(ii) $N_{1}, N_{4} \ll N$ and $N_{2}, N_{5} \sim N$,

(iii) $N_{1}, N_{5} \ll N$ and $N_{2}, N_{4} \sim N$.

(iv) $N_{2}, N_{4} \ll N$ and $N_{1}, N_{5} \sim N$,

(v) $N_{2}, N_{5} \ll N$ and $N_{1}, N_{4} \sim N$,

(vi) $N_{4}, N_{5} \ll N$ and $N_{1}, N_{2} \sim N$.

ID. $N_{3} \sim N, N_{6} \sim N$ and 1 low:

(i) $N_{1} \ll N$ and $N_{2}, N_{4}, N_{5} \sim N$,

(ii) $N_{2} \ll N$ and $N_{1}, N_{4}, N_{5} \sim N$,

(iii) $N_{4} \ll N$ and $N_{1}, N_{2}, N_{5} \sim N$,

(iv) $N_{5} \ll N$ and $N_{1}, N_{2}, N_{4} \sim N$.

IE. $N_{3} \sim N, N_{6} \sim N$ and $N_{1}, N_{2}, N_{4}, N_{5} \sim N$.

IIA. $N_{3} \sim N$ and $N_{6} \ll N$ and 3 lows:

(i) $N_{1}, N_{2}, N_{4} \ll N$ and $N_{5} \sim N$,

(ii) $N_{1}, N_{2}, N_{5} \ll N$ and $N_{4} \sim N$,

IIB. $N_{3} \sim N$ and $N_{6} \ll N$ and 2 lows:

(i) $N_{1}, N_{2} \ll N$ and $N_{4}, N_{5} \sim N$,

(ii) $N_{1}, N_{4} \ll N$ and $N_{2}, N_{5} \sim N$, 
(iii) $N_{1}, N_{5} \ll N$ and $N_{2}, N_{4} \sim N$,

(iv) $N_{2}, N_{4} \ll N$ and $N_{1}, N_{5} \sim N$.

(v) $N_{2}, N_{5} \ll N$ and $N_{1}, N_{4} \sim N$.

IIC. $N_{3} \sim N$ and $N_{6} \ll N$ and 1 low:
(i) $N_{1} \ll N$ and $N_{2}, N_{4}, N_{5} \sim N$,
(ii) $N_{2} \ll N$ and $N_{1}, N_{4}, N_{5} \sim N$,
(iii) $N_{4} \ll N$ and $N_{1}, N_{2}, N_{5} \sim N$,
(iv) $N_{5} \ll N$ and $N_{1}, N_{2}, N_{4} \sim N$.

IID. $N_{3} \sim N$ and $N_{6} \ll N$ and $N_{1}, N_{2}, N_{4}, N_{5} \sim N$.

IIIA. $N_{6} \sim N$ and $N_{3} \ll N$ and 3 lows:

(i) $N_{2}, N_{4}, N_{5} \ll N$ and $N_{1} \sim N$,

(ii) $N_{1}, N_{4}, N_{5} \ll N$ and $N_{2} \sim N$.

IIIB. $N_{6} \sim N$ and $N_{3} \ll N$ and 2 lows:

(i) $N_{4}, N_{5} \ll N$ and $N_{1}, N_{2} \sim N$,

(ii) $N_{1}, N_{4} \ll N$ and $N_{2}, N_{5} \sim N$,

(iii) $N_{1}, N_{5} \ll N$ and $N_{2}, N_{4} \sim N$,

(iv) $N_{2}, N_{4} \ll N$ and $N_{1}, N_{5} \sim N$,

(v) $N_{2}, N_{5} \ll N$ and $N_{1}, N_{4} \sim N$.

IIIC. $N_{6} \sim N$ and $N_{3} \ll N$ and 1 low:

(i) $N_{1} \ll N$ and $N_{2}, N_{4}, N_{5} \sim N$,

(ii) $N_{2} \ll N$ and $N_{1}, N_{4}, N_{5} \sim N$,

(iii) $N_{4} \ll N$ and $N_{1}, N_{2}, N_{5} \sim N$,

(iv) $N_{5} \ll N$ and $N_{1}, N_{2}, N_{4} \sim N$.

IIID. $N_{6} \sim N$ and $N_{3} \ll N$ and $N_{1}, N_{2}, N_{4}, N_{5} \sim N$.

IVA. $N_{3} \ll N, N_{6} \ll N$ and 2 lows:

(i) $N_{1}, N_{4} \ll N$ and $N_{2}, N_{5} \sim N$,

(ii) $N_{1}, N_{5} \ll N$ and $N_{2}, N_{4} \sim N$,

(iii) $N_{2}, N_{4} \ll N$ and $N_{1}, N_{5} \sim N$,

(iv) $N_{2}, N_{5} \ll N$ and $N_{1}, N_{4} \sim N$.

IVB. $N_{3} \ll N, N_{6} \ll N$ and 1 low:

(i) $N_{1} \ll N$ and $N_{2}, N_{4}, N_{5} \sim N$,

(ii) $N_{2} \ll N$ and $N_{1}, N_{4}, N_{5} \sim N$,

(iii) $N_{4} \ll N$ and $N_{1}, N_{4}, N_{5} \sim N$,

(iv) $N_{5} \ll N$ and $N_{1}, N_{2}, N_{4} \sim N$.

IVC. $N_{3} \ll N, N_{6} \ll N$ and $N_{1}, N_{2}, N_{4}, N_{5} \sim N$.

In what follows we will use the following estimates repeatedly:

Lemma 4.9. Let $w_{N_{i}}$ be as above. Then

$$
\begin{aligned}
\left\|w_{N_{i}}\right\|_{X^{0+, \frac{1}{2}-}} & \leq N_{i}^{-\frac{1}{2}+}\left\|w_{N_{i}}\right\|_{X_{3}^{\frac{2}{3}-, \frac{1}{2}-}}, \\
\left\|w_{N_{i}}\right\|_{X^{\frac{1}{2}-, \frac{1}{3}+}} & \leq\left\|w_{N_{i}}\right\|_{X_{3}^{\frac{2}{3}-, \frac{1}{2}-} \cdot} \cdot
\end{aligned}
$$


We also have

$$
\left\|w_{N_{i}}\right\|_{L_{x t}^{8}} \leq\left\|w_{N_{i}}\right\|_{X_{3}^{\frac{13}{24}+\frac{3}{8}+}}
$$

If we assume that $\sigma_{i} \lesssim N^{\gamma}$ for any $\gamma>0$, then

$$
\left\|w_{N_{i}}\right\|_{L_{x t}^{\infty}} \leq N^{0+}\left\|w_{N_{i}}\right\|_{X_{3}^{\frac{2}{3}-, \frac{1}{2}-}} \text {. }
$$

Proof. The estimates (4.23) and (4.24) are a consequence of frequency localization and Hölder's inequality. The estimate (4.26) is a consequence of Sobolev embedding together with the assumption that $\sigma_{i} \lesssim N^{\gamma}$.

Lemma 4.10. Let $0<\beta<2, \rho \geq 0$ and $\delta>0$. Let $M>0$ and $w_{M}$ be such that $\operatorname{supp} w_{M}(\cdot, x) \subset[-\delta, \delta], x \in \mathbb{T}$. Define

$$
\widehat{J_{\beta} w_{M}}(\tau, n):=\chi_{\{|n| \sim M\}} \chi_{\left\{\left|\tau+n^{2}\right| \leq M^{\beta}\right\}}\left|\widehat{w_{M}}(\tau, n)\right| .
$$

Then

$$
\left\|J_{\beta} w_{M}\right\|_{X^{0, \rho}} \lesssim C_{\delta} A(\beta, M)^{1 / 6} M^{\rho \beta+}\left\|w_{M}\right\|_{X_{3}^{0,1 / 6}}
$$

where $A(M, \beta)$ defined below is bounded by $1+M^{\beta-1}$.

Proof. We write

$$
\begin{aligned}
\left\|J_{\beta} w_{M}\right\|_{X^{0, \rho}}^{2} & =\sum_{|n| \sim M} \int_{\left|\tau+n^{2}\right| \leq M^{\beta}}\left|\widehat{w_{M}}(\tau, n)\right|^{2}\left\langle\tau+n^{2}\right\rangle^{2 \rho} d \tau \\
& \leq M^{2 \rho \beta} \int_{\tau}\left(\sum_{|n| \sim M,\left|\tau+n^{2}\right| \leq M^{\beta}}\left|\widehat{w_{M}}(\tau, n)\right|^{2}\right) d \tau \\
& \leq M^{2 \rho \beta} \int_{\tau}\left[\sum_{|n| \sim M,\left|\tau+n^{2}\right| \leq M^{\beta}}\left|\widehat{w_{M}}(\tau, n)\right|^{3}\right]^{2 / 3}|S(\tau, M, \beta)|^{1 / 3} d \tau
\end{aligned}
$$

where

$$
S(\tau, M, \beta):=\left\{n \in \mathbb{Z}:|n| \sim M \text { and }\left|\tau+n^{2}\right| \leq M^{\beta}\right\},
$$

and $|S|$ represents the counting measure of the set.

We will show below that

$$
A(M, \beta):=\sup _{\tau}|S(\tau, M, \beta)| \leq 1+M^{\beta-1} .
$$

Hence (4.27) is less than or equal to

$$
\begin{aligned}
& A(M, \beta)^{1 / 3} M^{2 \rho \beta} \int_{\tau}\left[\sum_{n} \chi_{\{|n| \sim M\}}(n) \chi_{\left\{\left|\tau+n^{2}\right| \leq M^{\beta}\right\}}(\tau, n)\left|\widehat{w_{M}}(\tau, n)\right|^{3}\right]^{2 / 3} d \tau \\
& =A(M, \beta)^{1 / 3} M^{2 \rho \beta} \int_{\tau}\left\|\left\{\chi_{\left\{\left|\tau+n^{2}\right| \leq M^{\beta}\right\}}(\tau, n) \widehat{w_{M}}(\tau, n)\right\}_{n}\right\|_{\ell^{3}(|n| \sim M)}^{2} d \tau \\
& \sim A(M, \beta)^{1 / 3} M^{2 \rho \beta} \int_{t}\left\|\mathcal{F}_{\tau}^{-1}\left(\left\{\chi_{\left\{\left|\tau+n^{2}\right| \leq M^{\beta}\right\}}(\tau, n) \widehat{w_{M}}(\tau, n)\right\}_{n}\right)(t)\right\|_{\ell^{3}(|n| \sim M)}^{2} d t \\
& =A(M, \beta)^{1 / 3} M^{2 \rho \beta} \int_{t}\left\|\left\{\mathcal{F}_{\tau}^{-1}\left(\chi_{\left\{\left|\tau+n^{2}\right| \leq M^{\beta}\right\}}(\tau, n)\right) * \mathcal{F}_{\tau}^{-1}\left(\widehat{w_{M}}(\tau, n)\right)\right\}_{n}(t)\right\|_{\ell^{3}(|n| \sim M)}^{2} d t
\end{aligned}
$$


Note that $\mathcal{F}_{\tau}^{-1}\left(\widehat{w_{M}}(\cdot, n)\right)(t)$ is still supported on $[-\delta, \delta]$ for all $n$ and

$$
\mathcal{F}_{\tau}^{-1}\left(\chi_{\left\{\left|\tau+n^{2}\right| \leq M^{\beta}\right\}}(\cdot, n)\right)(t)=2 e^{-i t n^{2}} \frac{\sin \left(M^{\beta} t\right)}{t} .
$$

We then continue the above chain of inequalities with

$$
\begin{aligned}
= & A(M, \beta)^{1 / 3} M^{2 \rho \beta} \\
& \times \int_{t}\left\|\int_{\mathbb{R}} \chi_{[-\delta, \delta]}\left(t^{\prime}\right) \mathcal{F}_{n}\left(w_{M}\left(t^{\prime}, \cdot\right)\right)(n) e^{-i\left(t-t^{\prime}\right) n^{2}} \frac{\sin \left(M^{\beta}\left(t-t^{\prime}\right)\right)}{t-t^{\prime}} d t^{\prime}\right\|_{\ell^{3}(|n| \sim M)}^{2} d t \\
\leq & A(M, \beta)^{1 / 3} M^{2 \rho \beta} \\
& \times \int_{\mathbb{R}}\left[\int_{\mathbb{R}} \chi_{[-\delta, \delta]}\left(t^{\prime}\right)\left\|\mathcal{F}_{n}\left(w_{M}\left(t^{\prime}, \cdot\right)\right)(n)\right\|_{\ell^{3}(|n| \sim M)}\left|\frac{\sin \left(M^{\beta}\left(t-t^{\prime}\right)\right)}{t-t^{\prime}}\right| d t^{\prime}\right]^{2} d t .
\end{aligned}
$$

Let $p=2-$ and $q=1+$; then we compute

$$
\begin{aligned}
\left\|\frac{\sin \left(M^{\beta}\left(t-t^{\prime}\right)\right)}{t-t^{\prime}}\right\|_{L_{t}^{q}} & =M^{\beta}\left(\int_{\mathbb{R}}\left|\frac{\sin \left(M^{\beta} t\right)}{t M^{\beta}}\right|^{q} d t\right)^{1 / q} \\
& =M^{\beta} M^{-\beta / q}\left(\int_{\mathbb{R}}\left|\frac{\sin (r)}{r}\right|^{q} d r\right)^{1 / q} \lesssim M^{0+} .
\end{aligned}
$$

On the other hand, for $1 / \gamma=1 / p-1 / 3$,

$$
\begin{aligned}
& \left\|\chi_{[-\delta, \delta]}(\cdot)\right\| \mathcal{F}_{n}\left(w_{M}(t, \cdot)\right)(n)\left\|_{\ell^{3}(|n| \sim M)}\right\|_{L_{t}^{p}}^{2} \lesssim \delta^{2 / \gamma}\|\| \mathcal{F}_{n}\left(w_{M}(t, \cdot)\right)(n)\left\|_{\ell^{3}(|n| \sim M)}\right\|_{L_{t}^{3}}^{2} \\
& \lesssim \delta^{2 / \gamma}\|\| e^{i t n^{2}} \mathcal{F}_{n}\left(w_{M}(t, \cdot)\right)(n)\left\|_{\ell^{3}(|n| \sim M)}\right\|_{L_{t}^{3}}^{2} \\
& =\delta^{2 / \gamma}\|\| e^{i t n^{2}} \mathcal{F}_{n}\left(w_{M}(t, \cdot)\right)(n)\left\|_{L_{t}^{3}}\right\|_{\ell^{3}(|n| \sim M)}^{2} \\
& \lesssim \delta^{2 / \gamma}\|\| e^{i t n^{2}} \mathcal{F}_{n}\left(w_{M}(t, \cdot)\right)(n)\left\|_{H_{t}^{1 / 6}}\right\|_{\ell^{3}(|n| \sim M)}^{2}=\delta^{2 / \gamma}\left\|w_{M}\right\|_{X_{3}^{0,1 / 6}}^{2},
\end{aligned}
$$

where we used the Sobolev theorem and the definition of $X_{r}^{s, b}$. Finally by Young's inequality, (4.29) and (4.30) we have the desired estimate.

It remains to show (4.28). We use an argument similar to [18]. For fixed $\tau$ let $S:=$ $S(\tau, M, \beta) \neq \emptyset$. Then there exists $n_{0} \in S$ and hence

$$
\begin{aligned}
|S| & \leq 1+\left|\left\{l \in \mathbb{Z}:\left|n_{0}+l\right| \sim M,\left|\tau+\left(n_{0}+l\right)^{2}\right| \leq M^{\beta}\right\}\right| \\
& \leq 1+\left|\left\{l \in \mathbb{Z}:|l| \leq M,\left|2 n_{0} l+l^{2}\right| \lesssim M^{\beta}\right\}\right| .
\end{aligned}
$$

We have $\left|2 n_{0} l+l^{2}\right|=\left|\left(l+n_{0}\right)^{2}-n_{0}^{2}\right| \lesssim M^{\beta}$ if and only if

$$
-C M^{\beta}+n_{0}^{2} \leq\left(l+n_{0}\right)^{2} \leq n_{0}^{2}+C M^{\beta} .
$$

Hence we need $|l| \leq M$ to satisfy

$$
\begin{gathered}
-\sqrt{n_{0}^{2}+C M^{\beta}} \leq l+n_{0} \leq \sqrt{n_{0}^{2}+C M^{\beta}} \\
l+n_{0} \geq \sqrt{n_{0}^{2}-C M^{\beta}} \quad \text { or } \quad l+n_{0} \leq-\sqrt{n_{0}^{2}-C M^{\beta}} .
\end{gathered}
$$


In other words we need to know the size of

$$
\left[-\sqrt{n_{0}^{2}+C M^{\beta}},-\sqrt{n_{0}^{2}-C M^{\beta}}\right] \cup\left[\sqrt{n_{0}^{2}-C M^{\beta}}, \sqrt{n_{0}^{2}+C M^{\beta}}\right]
$$

which is of the order of $M^{\beta} /\left|n_{0}\right|$. Hence since $\left|n_{0}\right| \sim M$ we have

$$
|S| \leq 1+M^{\beta-1}
$$

which implies (4.28) by taking $\sup _{\tau}$.

In what follows we are under the assumption that $\sigma_{j} \lesssim N^{7}$ for all $j=1, \ldots, 6$. Towards the end of the proof we remove this assumption. We begin by treating all cases with at least two high frequencies in the nonderivative terms. All cases in IC, ID, IE, IIB, IIC, IID, IIIB, IIIC, IIID, IVA, IVB, IVC follow from the following lemma applied with the exponent $\sigma$ set equal to 0 .

Lemma 4.11. Assume there are $i, j \in\{1,2,4,5\}$ such that $N_{i} \geq N^{1-\sigma}$ for $0 \leq \sigma<1 / 6$

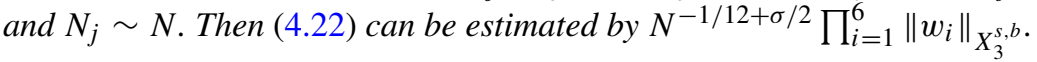

Proof. By Plancherel we see that (4.22) is less than or equal to

$$
\sum_{N_{j} \sim N ; N_{i} \geq N^{1-\sigma} ; N_{k} \leq N, 1 \leq k \leq 6} \int_{\mathbb{R}} \int_{\mathbb{T}} N_{3} N_{6} w_{N_{1}} w_{N_{2}} \overline{w_{N_{3}}} w_{N_{4}} \overline{w_{N_{5}} w_{N_{6}}} d x d t .
$$

Let $0<\beta<1$ to be determined below. Assume

$$
\sigma_{3} \leq N_{3}^{\beta} .
$$

By Cauchy-Schwarz's inequality, grouping the first three functions in (4.31) in $L_{x t}^{2}$ and the last three in $L_{x t}^{2}$ and using (2.3) we see that (4.31) is less than or equal to

$$
\sum_{N_{j} \sim N ; N_{i} \geq N^{1-\sigma} ; N_{k} \leq N} N_{3} N_{6} \prod_{i=1}^{6}\left\|w_{N_{i}}\right\|_{X^{\epsilon, \frac{1}{2}-}} .
$$

Note now that by (4.32), $w_{N_{3}}$ is equal to $J_{\beta} w_{N_{3}}$ as defined in Lemma 4.10. Then we have

$$
\left\|w_{N_{3}}\right\|_{X^{\epsilon, \frac{1}{2}-}} \leq C_{\delta} N_{3}^{1 / 2 \beta+}\left\|w_{N_{3}}\right\|_{X_{3}^{0, \frac{1}{6}+}} \cdot
$$

Hence by (4.23), (4.34) we deduce that (4.33) is less than or equal to

$$
\begin{aligned}
\sum_{N_{j} \sim N ; N_{i} \geq N^{1-\sigma} ; N_{k} \leq N} & N_{3} N_{6} N_{1}^{-\frac{1}{2}+} N_{2}^{-\frac{1}{2}+} N_{3}^{\frac{1}{2} \beta+} N_{3}^{-\frac{2}{3}} N_{4}^{-\frac{1}{2}+} N_{5}^{-\frac{1}{2}+} N_{6}^{-\frac{1}{2}+} \\
& \times\left(\prod_{i=1}^{6}\left\|w_{N_{i}}\right\|_{X_{3}^{\frac{2}{3}-, \frac{1}{2}-}}\right) . \\
& \lesssim \sum_{N_{j} \sim N ; N_{i} \geq N^{1-\sigma} ; N_{k} \leq N} N_{3}^{\frac{1}{3}+\frac{\beta}{2}+} N^{\frac{1}{2}+} N^{-1+\frac{\sigma}{2}}\left(\prod_{i=1}^{6}\left\|w_{N_{i}}\right\|_{X_{3}^{\frac{2}{3}-, \frac{1}{2}-}}\right) .
\end{aligned}
$$


Now we apply Hölder's inequality with $r=3, r^{\prime}=3 / 2$ to sum in $N_{j}, N_{i}, N_{k}$ (multiply and divide by $N_{j}^{-\epsilon}$ with a loss of $N^{\epsilon}$ for each term). For example,

$$
\sum_{N_{j} \leq N}\left\|w_{N_{j}}\right\|_{X_{3}^{s, b}}=\sum_{N_{j} \leq N}\|\|\left\langle n_{j}\right\rangle^{s}\left\langle\tau+n_{j}^{2}\right\rangle^{b} \widehat{w}_{N_{j}}\left(\tau, n_{j}\right)\left\|_{L_{\tau}^{2}}\right\|_{\ell^{3}} .
$$

Set $Y_{N_{j}}\left(n_{j}\right):=\left\|\left\langle n_{j}\right\rangle^{s}\left\langle\tau-n_{j}^{2}\right\rangle^{b} \widehat{w}_{N_{j}}\left(\tau, n_{j}\right)\right\|_{L_{\tau}^{2}}$. Then the expression in (4.35) equals

$$
\begin{aligned}
\sum_{N_{j} \leq N} N_{j}^{\varepsilon} N_{j}^{-\varepsilon}\left\|Y_{N_{j}}\right\|_{\ell^{3}} & \leq N^{\varepsilon}\left(\sum_{N_{j} \leq N} N_{j}^{-\frac{3}{2} \varepsilon}\right)^{2 / 3}\left(\sum_{N_{j} \leq N}\left\|Y_{N_{j}}\right\|_{\ell^{3}}^{3}\right)^{1 / 3} \\
& \lesssim N^{\varepsilon}\left(\sum_{N_{j} \leq N} \sum_{\left|n_{j}\right| \sim N_{j}}\left\|\left\langle n_{j}\right\rangle^{s}\left\langle\tau+n_{j}^{2}\right\rangle^{b} \widehat{w}_{j}\left(\tau, n_{j}\right)\right\|_{L_{\tau}^{2}}^{3}\right)^{1 / 3} \\
& \sim N^{\varepsilon}\left\|w_{j}\right\|_{X_{3}^{s, b}}
\end{aligned}
$$

Note then that all in all we get at worst a factor of $N^{-\frac{1}{6}+\frac{\beta}{2}+\frac{\sigma}{2}+}$.

Now assume that

$$
\sigma_{3} \geq N_{3}^{\beta}
$$

Then rewrite (4.31) as

$$
\sum_{N_{j} \sim N ; N_{i} \geq N^{1-\sigma} ; N_{k} \leq N} \int_{\mathbb{R}} \int_{\mathbb{T}} N_{3} N_{6}\left|\sigma_{3}\right|^{-\frac{1}{2}+} w_{N_{1}} w_{N_{2}}\left|\sigma_{3}\right|^{\frac{1}{2}-} \overline{w_{N_{3}}} w_{N_{4}} \overline{w_{N_{5}}} \overline{w_{N_{6}}} d x d t
$$

We do Hölder by placing $\left|\sigma_{3}\right|^{(1 / 2)-} \overline{w_{N_{3}}}$ in $L_{x t}^{2}$, the product of $\overline{w_{N_{6}}}$ with the two largest among $w_{N_{1}}, w_{N_{2}}, w_{N_{4}}, \bar{w}_{N_{5}}$ in $L_{x t}^{2}$, while the remaining ones in $L_{x t}^{\infty}$. Then by (4.23) and (4.26), we bound (4.36) by

$$
\begin{aligned}
& \lesssim \sum_{N_{j} \sim N ; N_{i} \geq N^{1-\sigma} ; N_{k} \leq N} N_{3} N_{3}^{-\frac{1}{2}-\frac{\beta}{2}+} N_{6} N_{6}^{-\frac{1}{2}+} N^{-\frac{1}{2}+} N^{-\frac{1}{2}+\frac{\sigma}{2}+}\left(\prod_{i=1}^{6}\left\|w_{N_{i}}\right\|_{X_{3}^{\frac{2}{3}-, \frac{1}{2}-}}\right) \\
& \lesssim \sum_{N_{j} \sim N ; N_{i} \geq N^{1-\sigma} ; N_{k} \leq N} N_{3}^{\frac{1}{2}-\frac{\beta}{2}+} N^{-\frac{1}{2}+\frac{\sigma}{2}+}\left(\prod_{i=1}^{6}\left\|w_{N_{i}}\right\| X_{X^{\frac{2}{3}-, \frac{1}{2}-}}\right) .
\end{aligned}
$$

We want that $\beta>\sigma$ to conclude by Hölder the desired inequality with a decay in $N$. We now impose that

$$
-\frac{1}{6}+\frac{\beta}{2}+\frac{\sigma}{2}=-\frac{\beta}{2}+\frac{\sigma}{2}
$$

whence $\beta=1 / 6$ and provided $0<\sigma<1 / 6$ the lemma follows.

It remains then to treat cases IA, IB, IIA and IIIA. Before starting we note the following support condition that will be used throughout what follows.

\section{Support condition}

By (4.15) and (4.16) the triplet $\left(w_{N_{1}}, w_{N_{2}}, \overline{w_{N_{3}}}\right)$ satisfies $n=n_{1}+n_{2}+n_{3},\left|n_{j}\right| \leq N$, $N \leq|n| \leq 3 N$ and $N \sim \max \left(N_{1}, N_{2}, N_{3}\right)$. 
Suppose that, say, $\max \left(N_{1}, N_{2}\right) \leq N^{\theta}$ for some $0<\theta<1$. Without any loss of generality assume $n>0$. Then $N \leq n \leq\left(n_{1}+n_{2}\right)+n_{3} \leq 2 N^{\theta}+N$ and hence $n=N+k$ where $0 \leq k \leq 2 N^{\theta}$. Next observe that $n_{3}=n-\left(n_{1}+n_{2}\right)=N+k-\left(n_{1}+n_{2}\right)$ with $\left|k-\left(n_{1}+n_{2}\right)\right| \leq 4 N^{\theta}$, whence $n_{3}=N+O\left(N^{\theta}\right)$. In other words, whenever $\max \left(N_{1}, N_{2}\right) \leq N^{\theta}$ the support of $\widehat{w_{N_{3}}}$ is of size $O\left(N^{\theta}\right)$. Note that we could have just as well said that the support of $\widehat{w_{N_{3}}}$ is of size $O\left(\max \left(N_{1}, N_{2}\right)\right)$ in lieu of $O\left(N^{\theta}\right)$.

When we are in this situation we say we have the support condition on $\overline{w_{N_{3}}}$. This argument is symmetric with respect to $w_{N_{1}}, w_{N_{2}}$ or $\overline{w_{N_{3}}}$. The exact same analysis holds for $\left(w_{N_{4}}, \overline{w_{N_{5}}}, \overline{w_{N_{6}}}\right)$. By abuse of notation we still write, for example, $\widehat{w_{N_{3}}}\left(n_{3}\right)$ for $\widehat{w_{N_{3}}}\left(n_{3}\right) \chi_{I_{3}}\left(n_{3}\right)$, where $I_{3}$ is the support of $\widehat{w_{N_{3}}}$ when the support condition holds.

Remark 4.12. As a consequence of the support condition, estimate (4.23) can be improved. For example if we have the support condition on $\widehat{w_{N_{3}}}$ then

$$
\left\|w_{N_{3}}\right\|_{X^{0+, \frac{1}{2}-}} \lesssim\left|I_{3}\right|^{1 / 6}\left\|w_{N_{3}}\right\|_{X_{3}^{0+, \frac{1}{2}-}} .
$$

Case IIIA. Note that (i) and (ii) are symmetric with respect to $j=1$ and $j=2$. So we only consider (i). Observe also that a priori there is no help from a large $\sigma_{j}$. Let $\sigma, \delta$ be two positive constants to be determined later but such that $1-\sigma>\delta$.

Subcase 1. Assume $N_{2}, N_{4}, N_{5}<N^{1-\sigma}, N_{3} \lesssim N^{\delta}$ and $N_{1} \sim N \sim N_{6}$ in (4.22). Then we have the support condition on $w_{N_{1}}$ and $\overline{w_{N_{6}}}$. Let us denote by $\sum_{*}$ the sum over the set of $N_{j} \leq N, 1 \leq j \leq 6$, such that $N_{1}, N_{6} \sim N, N_{j}<N^{1-\sigma}$ for $j=2,4,5$ and $N_{3} \lesssim N^{\delta}$. By Cauchy-Schwarz, (2.3), Lemma 4.9 and Remark 4.12 we then conclude that (4.22) is less than or equal to

$$
\begin{aligned}
& \sum_{*} N_{3} N_{6} \max \left(N_{2},\right.\left.N_{3}\right)^{1 / 6} N_{1}^{-\frac{2}{3}+} N_{2}^{-\frac{1}{2}+} N_{3}^{-\frac{1}{2}+} N_{4}^{-\frac{1}{2}+} N_{5}^{-\frac{1}{2}+} \\
& \times \max \left(N_{4}, N_{5}\right)^{1 / 6} N_{6}^{-\frac{2}{3}+}\left(\prod_{i=1}^{6}\left\|w_{N_{i}}\right\|_{X_{3}^{\frac{2}{3}-, \frac{1}{2}-}}\right) \\
& \lesssim \sum_{*} N_{3}^{\frac{1}{2}+} N_{6}^{\frac{1}{3}+} \max \left(N_{2}, N_{3}\right)^{1 / 6} N_{2}^{-\frac{1}{2}+} N^{-\frac{2}{3}+}\left(\prod_{i=1}^{6}\left\|w_{N_{i}}\right\|_{X_{3}^{\frac{2}{3}-, \frac{1}{2}-}}\right)
\end{aligned}
$$

since $N_{4}^{-(1 / 2)+} N_{5}^{-(1 / 2)+} \max \left(N_{4}, N_{5}\right)^{1 / 6}$ is bounded. On the other hand the latter expression is worst possible when $\max \left(N_{2}, N_{3}\right) \sim N_{3}$; hence if $\delta<1 / 2$ we conclude by Hölder as before with a decay of $N^{-1 / 3} N^{2 \delta / 3}$.

Subcase 2. Assume $N_{2}, N_{4}, N_{5}<N^{1-\sigma}, N_{3} \gtrsim N^{\delta}$ and $N_{1} \sim N \sim N_{6}$ in (4.22). We further subdivide as follows:

Subcase 2a. Assume $N_{2}, N_{4}, N_{5} \ll N^{\delta}, N_{3} \gtrsim N^{\delta}$ and $N_{1} \sim N \sim N_{6}$ in (4.22). Then from (4.20) there exists $\sigma_{j} \gtrsim N^{1+\delta}$. Denote by $\sum_{*}$ the sum over the set of $N_{j} \leq N$, $1 \leq j \leq 6$, such that $N_{1}, N_{6} \sim N, N_{j}<N^{\delta}$ for $j=2,4,5$ and $N_{3} \geq N^{\delta}$.

- Suppose $j=2,4$ or 5 ; $j=2$ or 4 are symmetric. So we treat first $j=2$ and then $j=5$. By Plancherel, (4.22) is less than or equal to 


$$
\begin{aligned}
\sum_{*} \int_{\mathbb{R}} \int_{\mathbb{T}} N_{3} N_{6} \sigma_{2}^{-\frac{1}{2}+} w_{N_{1}} \sigma_{2}^{\frac{1}{2}-} w_{N_{2}} \overline{w_{N_{3}}} w_{N_{4}} \overline{w_{N_{5}}} \overline{w_{N_{6}}} d x d t \\
\quad \lesssim \sum_{*} N_{3}^{\frac{1}{2}+} N_{6}^{\frac{1}{2}+} N^{-\frac{1}{2}-\frac{\delta}{2}} N_{1}^{-\frac{1}{2}+} N_{2}^{-\frac{1}{2}+} N_{4}^{0+} N_{5}^{0+}\left(\prod_{i=1}^{6}\left\|w_{N_{i}}\right\|_{X_{3}^{\frac{2}{3}-, \frac{1}{2}-}}\right)
\end{aligned}
$$

by Cauchy-Schwarz placing $w_{N_{1}} \overline{w_{N_{3}} w_{N_{6}}}$ in $L^{2}, \sigma_{2}^{1 / 2} w_{N_{2}}$ in $L^{2}$ and $w_{N_{4}} \overline{w_{N_{5}}}$ in $L^{\infty}$. From (2.3) and Lemma 4.9 we obtain the desired estimate with decay $N^{-\delta / 2}$ so long as $\delta>0$.

If $j=5$ we proceed as above with the same grouping in $L^{2}$ but exchanging the roles of $w_{N_{2}}$ and $\overline{w_{N_{5}}}$ for the other $L^{2}$ and one of the $L^{\infty}$ bounds.

- Suppose $j=3,6$ or $1 ; j=3$ or 6 are symmetric. So we treat first $j=3$ and then $j=1$. Proceeding as above from (4.22) we now have

$$
\begin{aligned}
\sum_{*} \int_{\mathbb{R}} \int_{\mathbb{T}} N_{3} N_{6} \sigma_{3}^{-\frac{1}{2}+} w_{N_{1}} w_{N_{2}} \sigma_{3}^{\frac{1}{2}-} \overline{w_{N_{3}}} w_{N_{4}} \overline{w_{N_{5}}} \overline{w_{N_{6}}} d x d t \\
\quad \lesssim \sum_{*} N_{3}^{\frac{1}{2}+} N_{6}^{\frac{1}{2}+} N^{-\frac{1}{2}-\frac{\delta}{2}} N_{1}^{-\frac{1}{2}+} N_{2}^{0+} N_{4}^{-\frac{1}{2}+} N_{5}^{0+}\left(\prod_{i=1}^{6}\left\|w_{N_{i}}\right\|_{X_{3}^{\frac{2}{3}-, \frac{1}{2}-}}\right)
\end{aligned}
$$

by Cauchy-Schwarz placing $w_{N_{1}} w_{N_{4}} \overline{w_{N_{6}}}$ in $L^{2}, \sigma_{3}^{(1 / 2)-} \overline{w_{N_{3}}}$ in $L^{2}$ and $w_{N_{2}} \overline{w_{N_{5}}}$ in $L^{\infty}$. We thus obtain the desired estimate as before with decay $N^{-\delta / 2}$ so long as $\delta>0$.

If $j=1$ then we group $\overline{w_{N_{3}}} w_{N_{4}} \overline{w_{N_{6}}}$ in $L^{2}, \sigma_{1}^{(1 / 2)-} w_{N_{1}}$ in $L^{2}$ and the other two in $L^{\infty}$ to reach the same estimate.

Subcase $2 b$. Suppose there exists $i \in\{2,4,5\}$ such that $N_{i} \gtrsim N^{\delta}$ and $N_{j} \ll N^{\delta}$ for $j \neq i$, and $i, j \in\{2,4,5\}$ while still $N_{3} \gtrsim N^{\delta}$ and $N_{1} \sim N \sim N_{6}$ in (4.22).

- Suppose $i=2$ first. Then we further split the sum over this set into three sums, $S_{1}, S_{2}$ and $S_{3}$ according to whether $N^{\delta} \lesssim N_{2} \ll N_{3}, N_{2} \sim N_{3}$ or $N_{2} \gg N_{3}$ respectively. When considering the sums over $S_{1}$ or $S_{3}$ we deduce from (4.20) that there exists $\sigma_{j} \gtrsim$ $N^{1+\delta}$ and hence the estimates for $S_{1}$ and $S_{3}$ follow exactly as those in Subcase 2a.

We then treat $S_{2}$. Since $N_{2} \sim N_{3}$ and $N_{2}<N^{1-\sigma}$, we also have $N_{3}<N^{1-\sigma}$, while $N_{4}, N_{5} \lesssim N^{\delta}$. Thus we have the support condition on $w_{N_{1}}$ and $\overline{w_{N_{6}}}$. Then from (4.22) by Cauchy-Schwarz, (2.3), Lemma 4.9 and Remark 4.12, grouping $w_{N_{1}} w_{N_{2}} \overline{w_{N_{3}}}$ in $L^{2}$ and $w_{N_{4}} \overline{w_{N_{5}}} \overline{w_{N_{6}}}$ and (4.23) we have

$$
\begin{aligned}
\sum_{S_{2}} N_{3} N_{6} \max \left(N_{2}, N_{3}\right)^{1 / 6} N_{1}^{-\frac{2}{3}+} N_{2}^{-\frac{1}{2}+} N_{3}^{-\frac{1}{2}+} N_{4}^{-\frac{1}{2}+} N_{5}^{-\frac{1}{2}+} & \quad \times \max \left(N_{4}, N_{5}\right)^{1 / 6} N_{6}^{-\frac{2}{3}+}\left(\prod_{i=1}^{6}\left\|w_{N_{i}}\right\|_{X_{3}^{\frac{2}{3}-, \frac{1}{2}-}}\right) \\
& \lesssim \sum_{S_{2}} N_{6}^{\frac{1}{3}+} \max \left(N_{2}, N_{3}\right)^{1 / 6} N_{1}^{-\frac{2}{3}+}\left(\prod_{i=1}^{6}\left\|w_{N_{i}}\right\|_{X_{3}^{\frac{2}{3}-, \frac{1}{2}-}}\right)
\end{aligned}
$$

since $N_{4}^{-(1 / 2)+} N_{5}^{-(1 / 2)+} \max \left(N_{4}, N_{5}\right)^{1 / 6}$ is bounded and $N_{2} \sim N_{3}$. Summing as usual, we get the desired estimate with decay $N^{-(1 / 6)+}$ regardless of $\sigma>0$. 
- Suppose $i=4$. Again, we further split the sum over this set into three sums, over $S_{1}, S_{2}$ and $S_{3}$, according now to whether $N^{\delta} \lesssim N_{4} \ll N_{3}, N_{4} \sim N_{3}$ or $N_{4} \gg N_{3}$ respectively. For the sums over $S_{1}$ or $S_{3}$, from (4.20) we have $\sigma_{j} \gtrsim N^{1+\delta}$ and hence the estimates for $S_{1}$ and $S_{3}$ follow exactly as those in Subcase 2a.

We then treat $S_{2}$. Since $N_{4} \sim N_{3}, N_{3}<N^{1-\sigma}$ while $N_{2}, N_{5} \lesssim N^{\delta}$, once again we have the support condition on $w_{N_{1}}$ and $\overline{w_{N_{6}}}$. Proceeding as before we have

$$
\begin{aligned}
& \sum_{S_{2}} N_{3} N_{6} \max \left(N_{2}, N_{3}\right)^{1 / 6} N_{1}^{-\frac{2}{3}+} N_{2}^{-\frac{1}{2}+} N_{3}^{-\frac{1}{2}+} N_{4}^{-\frac{1}{2}+} N_{5}^{-\frac{1}{2}+} \\
& \quad \times \max \left(N_{4}, N_{5}\right)^{1 / 6} N_{6}^{-\frac{2}{3}+}\left(\prod_{i=1}^{6}\left\|w_{N_{i}}\right\|_{X_{3}^{\frac{2}{3}-, \frac{1}{2}-}}\right) \\
& \vdots \sum_{S_{2}} N_{3}^{\frac{1}{2}+} N_{6} N_{3}^{1 / 6} N^{-\frac{2}{3}+} N_{2}^{-\frac{1}{2}+} N_{4}^{-\frac{1}{2}+\frac{1}{6}+} N_{5}^{-\frac{1}{2}+} N^{-\frac{2}{3}+}\left(\prod_{i=1}^{6}\left\|w_{N_{i}}\right\|_{X_{3}^{\frac{2}{3}-, \frac{1}{2}-}}\right) \\
& \lesssim \sum_{S_{2}} N_{3}^{\frac{1}{3}+} N N^{-\frac{4}{3}+}\left(\prod_{i=1}^{6}\left\|w_{N_{i}}\right\|_{X_{3}^{\frac{2}{3}-, \frac{1}{2}-}}\right) .
\end{aligned}
$$

Since $N_{4} \sim N_{3}$ and $N_{3}<N^{1-\sigma}$, summing as before we have the desired estimate with decay $N^{-\sigma / 3}$ so long as $\sigma>0$.

- Suppose $i=5$. We split the sum over this set into three sums, over $S_{1}, S_{2}$ and $S_{3}$, according to whether $N^{\delta} \lesssim N_{5} \ll N_{3}, N_{5} \sim N_{3}$ or $N_{5} \gg N_{3}$ respectively. Again for the sums over $S_{1}$ or $S_{3}$, from (4.20) we have $\sigma_{j} \gtrsim N^{1+\delta}$ and hence the estimates for $S_{1}$ and $S_{3}$ follow exactly as those in Subcase 2a.

We then treat $S_{2}$. Since $N_{5} \sim N_{3}, N_{3}<N^{1-\sigma}$ while $N_{2}, N_{4} \lesssim N^{\delta}$, we have the support condition on $w_{N_{1}}$ and $\overline{w_{N_{6}}}$. Proceeding as before we have

$$
\begin{aligned}
\sum_{S_{2}} N_{3} N_{6} \max \left(N_{2}, N_{3}\right)^{1 / 6} N_{1}^{-\frac{2}{3}+} N_{2}^{-\frac{1}{2}+} N_{3}^{-\frac{1}{2}+} N_{4}^{-\frac{1}{2}+} N_{5}^{-\frac{1}{2}+} & \quad \times \max \left(N_{4}, N_{5}\right)^{1 / 6} N_{6}^{-\frac{2}{3}+}\left(\prod_{i=1}^{6}\left\|w_{N_{i}}\right\|_{X_{3}^{\frac{2}{3}-, \frac{1}{2}-}}\right) \\
& \lesssim \sum_{S_{2}} N_{3}^{\frac{1}{2}+} N_{6}^{\frac{1}{3}+} N_{3}^{1 / 6} N^{-\frac{2}{3}+} N_{2}^{-\frac{1}{2}+} N_{4}^{-\frac{1}{2}+} N_{5}^{-\frac{1}{2}+\frac{1}{6}+}\left(\prod_{i=1}^{6}\left\|w_{N_{i}}\right\|_{X_{3}^{\frac{2}{3}-, \frac{1}{2}-}}\right) \\
\lesssim & \sum_{S_{2}} N_{3}^{\frac{1}{3}+} N^{-\frac{1}{3}+}\left(\prod_{i=1}^{6}\left\|w_{N_{i}}\right\|_{X_{3}^{\frac{2}{3}-, \frac{1}{2}-}}\right)
\end{aligned}
$$

which gives the desired estimate with the same $N^{-\sigma / 3}$ decay as in the previous case so long as $\sigma>0$.

Subcase 2c. Suppose that there exist at least $i, j \in\{2,4,5\}(i \neq j)$ such that $N_{i}, N_{j} \gtrsim$ $N^{\delta}$ while $N_{3} \gtrsim N^{\delta}$ and $N_{1} \sim N \sim N_{6}$ in (4.22). Note that $N_{4}, N_{5}<N^{1-\sigma}$, which ensures the support condition on $\overline{w_{N_{6}}}$. 
- Suppose $(i, j)=(4,5)$. Proceeding as above and using similar arguments we have

$$
\begin{gathered}
\sum_{*} N_{3} N_{6} N_{1}^{-\frac{1}{2}+} N_{2}^{-\frac{1}{2}+} N_{3}^{-\frac{1}{2}+} N_{4}^{-\frac{1}{2}+} N_{5}^{-\frac{1}{2}+} \max \left(N_{4}, N_{5}\right)^{1 / 6} N_{6}^{-\frac{2}{3}+}\left(\prod_{i=1}^{6}\left\|w_{N_{i}}\right\|_{X_{3}^{\frac{2}{3}-, \frac{1}{2}-}}\right) \\
=\sum_{*} N_{3}^{\frac{1}{2}+} N_{6}^{\frac{1}{3}+} N^{-\frac{1}{2}+} N_{4}^{-\frac{1}{2}+} N_{5}^{-\frac{1}{2}+} \max \left(N_{4}, N_{5}\right)^{1 / 6}\left(\prod_{i=1}^{6}\left\|w_{N_{i}}\right\|_{X_{3}^{\frac{2}{3}-, \frac{1}{2}-}}\right),
\end{gathered}
$$

from which using that $N_{4}, N_{5} \gtrsim N^{\delta}$ and $N_{3} \gtrsim N^{\delta}$ we get the desired bound with decay $N^{1 / 3-5 \delta / 6}$ so long as $\delta>2 / 5$.

- Suppose $(i, j)=(2,5)$. Once again proceeding as before and using similar arguments we have

$$
\begin{aligned}
\sum_{*} N_{3} N_{6} N_{1}^{-\frac{1}{2}+} N_{2}^{-\frac{1}{2}+} N_{3}^{-\frac{1}{2}+} & N_{4}^{-\frac{1}{2}+} N_{5}^{-\frac{1}{2}+} \max \left(N_{4}, N_{5}\right)^{1 / 6} N_{6}^{-\frac{2}{3}+}\left(\prod_{i=1}^{6}\left\|w_{N_{i}}\right\|_{X_{3}^{\frac{2}{3}-, \frac{1}{2}-}}\right) \\
& \lesssim \sum_{*} N_{3}^{\frac{1}{2}+} N_{6}^{\frac{1}{3}+} N^{-\frac{1}{2}+} N^{-\frac{\delta}{2}} N^{-\frac{\delta}{2}+\frac{\delta}{6}}\left(\prod_{i=1}^{6}\left\|w_{N_{i}}\right\|_{X_{3}^{\frac{2}{3}-, \frac{1}{2}-}}\right)
\end{aligned}
$$

using that $N_{2} \gtrsim N^{\delta}$ and that $N_{4}^{-(1 / 2)+} N_{5}^{-(1 / 2)+} \max \left(N_{4}, N_{5}\right)^{1 / 6}$ is worse possible when $N_{4} \ll N_{5}$ but $N_{5} \gtrsim N^{\delta}$. Hence we once again obtain the desired estimate with decay $N^{1 / 3-5 \delta / 6}$ so long as $\delta>2 / 5$.

- Suppose $(i, j)=(2,4)$. This is exactly as in the previous case by exchanging the roles of 4 and 5 .

Subcase 3. Assume there exists at least one $i \in\{2,4,5\}$ such that $N_{i} \gtrsim N^{1-\sigma}$, $N_{2}, N_{4}, N_{5} \ll N$ while $N_{3} \ll N$ and $N_{1} \sim N \sim N_{6}$ in (4.22). This case follows from Lemma 4.11 with $0<\sigma<1 / 6$ as in its statement.

All in all, for Case IIIA we need $2 / 5<\delta<1 / 2$ and $0<\sigma<1 / 6$.

Remark 4.13. In the proof of the remaining cases, in order to keep the notation lighter, we will ignore the $+\epsilon$ appearing in the exponent of the $N_{i}$ 's in (4.23). For example we simply write $N_{i}^{-1 / 2}$ instead of $N_{i}^{-(1 / 2)+}$.

Case IA. Assume $N_{3} \sim N \sim N_{6}$ while $N_{1}, N_{2}, N_{4}, N_{5} \ll N$ in (4.22) and denote as before by $\sum_{*}$ the sum over this set. Observe that from (4.17)-(4.21) there exists $\sigma_{j} \gtrsim N^{2}$.

Subcase 1. Assume in addition $N_{1}, N_{2}<N^{\delta}$ for some $\delta>0$. We then have the support condition on $\overline{w_{N_{3}}}$.

- Suppose $j=3$ or 6 ; say $j=3$ ( $j=6$ is similar). Then we rewrite (4.22) as follows:

$$
\begin{aligned}
& \sum_{*} \int_{\mathbb{R}} \int_{\mathbb{T}} N^{2} \sigma_{3}^{-\frac{1}{2}+} w_{N_{1}} w_{N_{2}} \sigma_{3}^{\frac{1}{2}-} \overline{w_{N_{3}}} w_{N_{4}} \overline{w_{N_{5}}} \overline{w_{N_{6}}} d x d t \\
& \lesssim \sum_{*} N^{2} N^{-1} N_{1}^{0+} N_{2}^{0+} \max \left(N_{1}, N_{2}\right)^{1 / 6} N_{3}^{-2 / 3} N_{4}^{-1 / 2} N_{5}^{-1 / 2} N_{6}^{-1 / 2}\left(\prod_{i=1}^{6}\left\|w_{N_{i}}\right\|_{X_{3}^{\frac{2}{3}-, \frac{1}{2}-}}\right)
\end{aligned}
$$


by placing $\sigma_{3}^{(1 / 2)-} \overline{w_{N_{3}}}$ in $L_{x t}^{2}, w_{N_{4}} \overline{w_{N_{5}}} \overline{w_{N_{6}}}$ in $L_{x t}^{2}, w_{N_{1}} w_{N_{2}}$ in $L_{x t}^{\infty}$ and using the support condition on $\overline{w_{N}}$. By Hölder's inequality, summing as above, we get the desired estimate with decay $N^{\delta / 6-1 / 6}$ so long as $\delta<1$.

- Suppose $j=1,2,4$ or 5 . By symmetry (relative to conjugates) $j=1,2,4$ are similar; so suppose $j=1$. We rewrite (4.22) as

$$
\begin{aligned}
& \sum_{*} \int_{\mathbb{R}} \int_{\mathbb{T}} N^{2} \sigma_{1}^{-\frac{1}{2}+} w_{N_{1}} \sigma_{1}^{\frac{1}{2}-} w_{N_{2}} \overline{w_{N_{3}}} w_{N_{4}} \overline{w_{N_{5}}} \overline{w_{N_{6}}} d x d t \\
& \lesssim \sum_{*} N^{2} N^{-1} N_{1}^{-1 / 2} N_{2}^{0+} \max \left(N_{1}, N_{2}\right)^{1 / 6} N_{3}^{-2 / 3} N_{4}^{-1 / 2} N_{5}^{0+} N_{6}^{-1 / 2}\left(\prod_{i=1}^{6}\left\|w_{N_{i}}\right\|_{X_{3}^{\frac{2}{3}-, \frac{1}{2}-}}\right)
\end{aligned}
$$

by placing $\sigma_{1}^{(1 / 2)-} w_{N_{1}}$ in $L_{x t}^{2}, \overline{w_{N_{3}}} w_{N_{4}} \overline{w_{N_{6}}}$ in $L_{x t}^{2}, w_{N_{2}} \overline{w_{N_{5}}}$ in $L_{x t}^{\infty}$ and using the support condition on $\overline{w_{N_{3}}}$. Once again, by Hölder's inequality, summing as before we get the desired estimate with decay $N^{\delta / 6-1 / 6}$ so long as $\delta<1$.

If $j=5$, then

$$
\begin{aligned}
\sum_{*} & \int_{\mathbb{R}} \int_{\mathbb{T}} N^{2} \sigma_{5}^{-\frac{1}{2}+} w_{N_{1}} w_{N_{2}} \overline{w_{N_{3}}} w_{N_{4}} \sigma_{5}^{\frac{1}{2}-} \overline{w_{N_{5}}} \overline{w_{N_{6}}} d x d t \\
& \lesssim \sum_{*} N^{2} N^{-1} N_{1}^{0+} N_{2}^{0+} \max \left(N_{1}, N_{2}\right)^{1 / 6} N_{3}^{-\frac{2}{3}} N_{4}^{-\frac{1}{2}} N_{5}^{-\frac{1}{2}} N_{6}^{-\frac{1}{2}}\left(\prod_{i=1}^{6}\left\|w_{N_{i}}\right\|_{X_{3}^{\frac{2}{3}-, \frac{1}{2}-}}\right)
\end{aligned}
$$

by placing $\sigma_{5}^{(1 / 2)-} \overline{w_{N_{5}}}$ in $L_{x t}^{2}, \overline{w_{N_{3}}} w_{N_{4}} \overline{w_{N_{6}}}$ in $L_{x t}^{2}, w_{N_{1}} w_{N_{2}}$ in $L_{x t}^{\infty}$ and using the support condition on $\overline{w_{N_{3}}}$. Once again, by Hölder's inequality, summing as before we get the desired estimate with decay $N^{\delta / 6-1 / 6}$ so long as $0<\delta<1$.

Subcase 2. Assume either $N_{1}$ or $N_{2}$ is $>N^{\delta}$. Suppose $N_{1}>N^{\delta}$; otherwise exchange the roles of $w_{N_{1}}$ and $w_{N_{2}}$ below. We no longer rely on the support condition but on the lower bound on $N_{1}$ as follows.

- Suppose $j=3$ or 6 ; say $j=3$ ( $j=6$ is similar). Then proceeding as before we rewrite (4.22) as

$$
\begin{aligned}
\sum_{*} \int_{\mathbb{R}} \int_{\mathbb{T}} N^{2} \sigma_{3}^{-\frac{1}{2}+} w_{N_{1}} w_{N_{2}} \sigma_{3}^{\frac{1}{2}-} \overline{w_{N_{3}}} w_{N_{4}} \overline{w_{N_{5}}} \overline{w_{N_{6}}} d x d t \\
\quad \lesssim \sum_{*} N^{2} N^{-1} N_{1}^{-1 / 2} N_{2}^{0+} N_{3}^{-1 / 2} N_{4}^{0+} N_{5}^{-1 / 2} N_{6}^{-1 / 2}\left(\prod_{i=1}^{6}\left\|w_{N_{i}}\right\|_{X_{3}^{\frac{2}{3}-, \frac{1}{2}-}}\right)
\end{aligned}
$$

by placing $\sigma_{3}^{(1 / 2)-} \overline{w_{N_{3}}}$ in $L_{x t}^{2}, w_{N_{1}} \overline{w_{N_{5}}} \overline{w_{N_{6}}}$ in $L_{x t}^{2}, w_{N_{2}} w_{N_{4}}$ in $L_{x t}^{\infty}$. By Hölder's inequality, summing as above, we get the desired estimate with decay $N^{-\delta / 2}$ so long as $\delta>0$.

- Suppose $j=1$ or 2 ; say $j=1$ ( $j=2$ is similar). We now write

$$
\begin{aligned}
\sum_{*} \int_{\mathbb{R}} \int_{\mathbb{T}} N^{2} \sigma_{1}^{-\frac{1}{2}+} w_{N_{1}} \sigma_{1}^{\frac{1}{2}-} w_{N_{2}} \overline{w_{N_{3}}} w_{N_{4}} \overline{w_{N_{5}}} \overline{w_{N_{6}}} d x d t \\
\quad \lesssim \sum_{*} N^{2} N^{-1} N_{1}^{-1 / 2} N_{2}^{-1 / 2} N_{3}^{-1 / 2} N_{4}^{0+} N_{5}^{0+} N_{6}^{-1 / 2}\left(\prod_{i=1}^{6}\left\|w_{N_{i}}\right\|_{X_{3}^{\frac{2}{3}-, \frac{1}{2}-}}\right)
\end{aligned}
$$


by placing $\sigma_{1}^{(1 / 2)-} w_{N_{1}}$ in $L_{x t}^{2}, w_{N_{2}} \overline{w_{N_{3}}} \overline{w_{N_{6}}}$ in $L_{x t}^{2}, w_{N_{4}} \overline{w_{N_{5}}}$ in $L_{x t}^{\infty}$. Once again, by Hölder's inequality and summing as above, we get the desired estimate with decay $N^{-\delta / 2}$ so long as $\delta>0$.

- Suppose $j=4$. Then proceed as above but place $\sigma_{4}^{(1 / 2)-} w_{N_{4}}$ in $L_{x t}^{2}, w_{N_{1}} \overline{w_{N_{3}} w_{N_{6}}}$ in $L_{x t}^{2}$, and $w_{N_{2}} \overline{w_{N_{5}}}$ in $L_{x t}^{\infty}$.

- Suppose $j=5$. Then once again we proceed as above but now place $\sigma_{5}^{(1 / 2)-} \overline{w_{N_{5}}}$ in $L_{x t}^{2}, w_{N_{1}} \overline{w_{N_{3}}} \overline{w_{N_{6}}}$ in $L_{x t}^{2}$, and $w_{N_{2}} w_{N_{4}}$ in $L_{x t}^{\infty}$.

Remark 4.14. Matching Subcases 1 and 2 above means $-\delta / 2=\delta / 6-1 / 6$, which requires $\delta=1 / 4$ and yields a decay of $N^{-(1 / 8)+}$.

Case IIA. Part (i) will follow similarly to Case IA while part (ii) to Case IIIA.

Part (i). We are under the assumptions $N_{3} \sim N \sim N_{5}$ while $N_{1}, N_{2}, N_{4}, N_{6} \ll N$. It follows from (4.21) that there exists $\sigma_{j} \gtrsim N^{2}$. We proceed exactly as in IA exchanging in each instance the roles of $\overline{w_{N_{6}}}$ and $\overline{w_{N_{5}}}$.

Part (ii). We are under the assumptions $N_{3} \sim N \sim N_{4}$ while $N_{1}, N_{2}, N_{5}, N_{6} \ll N$. We have a priori no help from a large $\sigma_{j}$ at our disposal. We then proceed as in IIIA above with the role of $\left(N_{3} ; \overline{w_{N_{3}}}\right)$ switched with that of $\left(N_{6} ; \overline{w_{N_{6}}}\right)$, and $\left(N_{1} ; w_{N_{1}}\right)$ with $\left(N_{4} ; w_{N_{4}}\right)$. Hence for $\sigma, \delta>0$ to be determined, in Subcase 1 we are under the assumption $N_{1}, N_{2}, N_{5}<N^{1-\sigma}, N_{6} \lesssim N^{\delta}$ and $N_{3} \sim N \sim N_{4}$. In Subcase 2 we assume that $N_{1}, N_{2}, N_{5}<N^{1-\sigma}$ while $N_{6} \gtrsim N^{\delta}$ and $N_{3} \sim N \sim N_{4}$, and further subdivide just as before into Subcase $2 \mathrm{a}: N_{1}, N_{2}, N_{5} \ll N^{\delta}$ while $N_{6} \gtrsim N^{\delta}$ which implies from (4.19) the existence of a $\sigma_{j} \gtrsim N^{1+\delta}$; Subcase 2b: there exists $i \in\{1,2,5\}$ such that $N_{i} \gg N^{\delta}$ and $N_{j} \lesssim N^{\delta}$ for $j \neq i$ and $i, j \in\{1,2,5\}$ while still $N_{6} \gtrsim N^{\delta}$ and $N_{3} \sim N \sim N_{4}$ in (4.22), and Subcase 2c: there exist at least $i, j \in\{1,2,5\}(i \neq j)$ such that $N_{i}, N_{j} \gg N^{\delta}$ while $N_{6} \gtrsim N^{\delta}$ and $N_{3} \sim N \sim N_{4}$ in (4.22). Note that $N_{1}, N_{2}<N^{1-\sigma}$, which ensures the support condition on $\overline{w_{N_{3}}}$. Subcase 3: Assume there exists at least one $i \in\{1,2,5\}$ such that $N_{i} \gtrsim N^{1-\sigma}, N_{2}, N_{1}, N_{5} \ll N$ while $N_{6} \ll N$ and $N_{3} \sim N \sim N_{4}$ in (4.22). This case follows from Lemma 4.11 with $0<\sigma<1 / 6$ as in its statement.

Proceeding then just as in IIIA we deduce the desired estimate with the same decay in $N$ as in IIIA as long as $2 / 5<\delta<1 / 2$ and $0<\sigma<1 / 6$ as before.

Case IB. We first note that parts (ii), (iii) and (iv) are all symmetric relative to conjugation; so we only consider (i) and (ii).

Part (i). We are under the assumptions $N_{3} \sim N_{5} \sim N_{6} \sim N$ while $N_{1}, N_{2}, N_{4} \ll N$. It follows from (4.21) that there exists $\sigma_{j} \gtrsim N^{2}$.

- Suppose $j=1,2$ or 4 . By symmetry it is enough to consider $j=1$ and $j=4$. To obtain decay we need to use the support condition. Thus we further subdivide into two cases. 
Subcase 1. Assume in addition $N_{1}, N_{2}<N^{\delta}$ for some $\delta>0$. We then have the support condition on $\overline{w_{N_{3}}}$. For $j=1$ we have

$$
\begin{aligned}
& \sum_{*} \int_{\mathbb{R}} \int_{\mathbb{T}} N^{2} \sigma_{1}^{-\frac{1}{2}+} w_{N_{1}} \sigma_{1}^{\frac{1}{2}-} w_{N_{2}} \overline{w_{N_{3}}} w_{N_{4}} \overline{w_{N_{5}}} \overline{w_{N_{6}}} d x d t \\
& \quad \lesssim \sum_{*} N^{2} N^{-1} N_{1}^{-1 / 2} N_{2}^{-1 / 2} N_{3}^{-2 / 3} N^{\delta / 6} N_{4}^{0+} N_{5}^{0+} N_{6}^{-1 / 2}\left(\prod_{i=1}^{6}\left\|w_{N_{i}}\right\|_{X_{3}^{\frac{2}{3}-, \frac{1}{2}-}}\right)
\end{aligned}
$$

by placing $\sigma_{1}^{(1 / 2)-} w_{N_{1}}$ in $L_{x t}^{2}, w_{N_{2}} \overline{w_{N_{3}}} \overline{w_{N_{6}}}$ in $L_{x t}^{2}, w_{N_{4}} \overline{w_{N_{5}}}$ in $L_{x t}^{\infty}$. By Hölder's inequality, summing as above, we get the desired estimate with decay $N^{-1 / 6+\delta / 6}$ so long as $0<\delta<1$.

For $j=4$, we place $\sigma_{4}^{1 / 2} w_{N_{4}}$ in $L_{x t}^{2}, w_{N_{1}} \overline{w_{N_{3}} w_{N_{6}}}$ in $L_{x t}^{2}$ and $w_{N_{2}} \overline{w_{N_{5}}}$ in $L_{x t}^{\infty}$ and proceed similarly.

Subcase 2. Assume either $N_{1}$ or $N_{2}$ is $>N^{\delta}$. By symmetry suppose $N_{1}>N^{\delta}$; otherwise exchange the roles of $w_{N_{1}}$ and $w_{N_{2}}$ below. We use then the lower bound on $N_{1}$ as follows. For $j=1$,

$$
\begin{aligned}
\sum_{*} \int_{\mathbb{R}} & \int_{\mathbb{T}} N^{2} \sigma_{1}^{-\frac{1}{2}+} w_{N_{1}} \sigma_{1}^{\frac{1}{2}-} w_{N_{2}} \overline{w_{N_{3}}} w_{N_{4}} \overline{w_{N_{5}}} \overline{w_{N_{6}}} d x d t \\
& \lesssim \sum_{*} N^{2} N^{-1} N_{1}^{-1 / 2} N_{2}^{-1 / 2} N_{3}^{-1 / 2} N_{4}^{0+} N_{5}^{0+} N_{6}^{-1 / 2}\left(\prod_{i=1}^{6}\left\|w_{N_{i}}\right\|_{X_{3}^{\frac{2}{3}-, \frac{1}{2}-}}\right)
\end{aligned}
$$

by placing $\sigma_{1}^{(1 / 2)-} w_{N_{1}}$ in $L_{x t}^{2}, w_{N_{2}} \overline{w_{N_{3}}} \overline{w_{N_{6}}}$ in $L_{x t}^{2}, w_{N_{4}} \overline{w_{N_{5}}}$ in $L_{x t}^{\infty}$. Hence, by Hölder's inequality and summing as usual we get the desired estimate with decay $N^{-\delta / 2}$ so long as $\delta>0$.

For $j=4$, we place $\sigma_{4}^{(1 / 2)-} w_{N_{4}}$ in $L_{x t}^{2}, w_{N_{1}} \overline{w_{N_{3}}} \overline{w_{N_{6}}}$ in $L_{x t}^{2}$ and $w_{N_{2}} \overline{w_{N_{5}}}$ in $L_{x t}^{\infty}$ and proceed similarly.

Remark 4.15. Note that once again, matching Subcases 1 and 2 above means $-\delta / 2=$ $\delta / 6-1 / 6$, which requires $\delta=1 / 4$ and yields a decay of $N^{-(1 / 8)+}$.

- Suppose $j=3,6$ or 5. By symmetry relative to conjugation it is enough to consider $j=3$. We have

$$
\begin{aligned}
\sum_{*} \int_{\mathbb{R}} & \int_{\mathbb{T}} N^{2} \sigma_{3}^{-\frac{1}{2}+} w_{N_{1}} w_{N_{2}} \sigma_{3}^{\frac{1}{2}-} \overline{w_{N_{3}}} w_{N_{4}} \overline{w_{N_{5}}} \overline{w_{N_{6}}} d x d t \\
& \lesssim \sum_{*} N^{2} N^{-1} N_{1}^{0+} N_{2}^{0+} N_{3}^{-1 / 2} N_{4}^{-1 / 2} N_{5}^{-1 / 2} N_{6}^{-1 / 2}\left(\prod_{i=1}^{6}\left\|w_{N_{i}}\right\|_{X_{3}^{\frac{2}{3}-, \frac{1}{2}-}}\right)
\end{aligned}
$$

by placing $\sigma_{3}^{(1 / 2)-} \overline{w_{N_{3}}}$ in $L_{x t}^{2}, w_{N_{4}} \overline{w_{N_{5}}} \overline{w_{N_{6}}}$ in $L_{x t}^{2}, w_{N_{1}} w_{N_{2}}$ in $L_{x t}^{\infty}$. Hence, by Hölder's inequality, summing as usual we get the desired estimate with decay $N^{-(1 / 2)+}$. 
Part (ii). We are under the assumptions $N_{3} \sim N_{4} \sim N_{6} \sim N$ while $N_{1}, N_{2}, N_{5} \ll N$. It follows from (4.19) that there exists $\sigma_{j} \gtrsim N^{2}$.

- Suppose $j=1,2$ or 5. If $j=1$ then

$$
\begin{aligned}
\sum_{*} \int_{\mathbb{R}} \int_{\mathbb{T}} N^{2} \sigma_{1}^{-\frac{1}{2}+} \sigma_{1}^{\frac{1}{2}-} w_{N_{1}} w_{N_{2}} \overline{w_{N_{3}}} w_{N_{4}} \overline{w_{N_{5}}} \overline{w_{N_{6}}} d x d t \\
\quad \lesssim \sum_{*} N^{2} N^{-1} N_{1}^{-1 / 2} N_{2}^{0+} N_{3}^{-1 / 2} N_{4}^{-1 / 2} N_{5}^{0+} N_{6}^{-1 / 2}\left(\prod_{i=1}^{6}\left\|w_{N_{i}}\right\|_{X_{3}^{\frac{2}{3}-, \frac{1}{2}-}}\right)
\end{aligned}
$$

by placing $\sigma_{1}^{(1 / 2)-} w_{N_{1}}$ in $L_{x t}^{2}, w_{N_{4}} \overline{w_{N_{3}}} \overline{w_{N_{6}}}$ in $L_{x t}^{2}, w_{N_{2}} \overline{w_{N_{5}}}$ in $L_{x t}^{\infty}$. Hence, by Hölder's inequality and summing as usual we get the desired estimate with decay $N^{-(1 / 2)+}$.

If $j=2,5$ we proceed similarly, keeping $w_{N_{4}} \overline{w_{N_{3}}} \overline{w_{N_{6}}}$ in $L_{x t}^{2}$ and exchanging the roles of either $w_{N_{2}}$ or $\overline{w_{N_{5}}}$ with that of $w_{N_{1}}$ above.

- Suppose $j=3,6$ or 4 . If $j=3$ then

$$
\begin{aligned}
\sum_{*} \int_{\mathbb{R}} \int_{\mathbb{T}} N^{2} \sigma_{3}^{-\frac{1}{2}+} w_{N_{1}} w_{N_{2}} \sigma_{3}^{\frac{1}{2}-} \overline{w_{N_{3}}} w_{N_{4}} \overline{w_{N_{5}}} \overline{w_{N_{6}}} d x d t \\
\quad \lesssim \sum_{*} N^{2} N^{-1} N_{1}^{0+} N_{2}^{-1 / 2} N_{3}^{-1 / 2} N_{4}^{-1 / 2} N_{5}^{0+} N_{6}^{-1 / 2}\left(\prod_{i=1}^{6}\left\|w_{N_{i}}\right\|_{X_{3}^{\frac{2}{3}-, \frac{1}{2}-}}\right)
\end{aligned}
$$

by placing $\sigma_{3}^{(1 / 2)-} \overline{w_{N_{3}}}$ in $L_{x t}^{2}, w_{N_{2}} w_{N_{4}} \overline{w_{N_{6}}}$ in $L_{x t}^{2}, w_{N_{1}} \overline{w_{N_{5}}}$ in $L_{x t}^{\infty}$. Hence, by Hölder's and summing as usual we get the desired estimate with decay $N^{-(1 / 2)+}$.

If $j=6$ we proceed similarly exchanging the roles of $\overline{w_{N_{3}}}$ and $\overline{w_{N_{6}}}$ above.

If $j=4$ we place $\sigma_{4}^{(1 / 2)-} w_{N_{4}}$ in $L_{x t}^{2}$ and group $w_{N_{2}} \overline{w_{N_{3}}} \overline{w_{N_{6}}}$ in $L_{x t}^{2}$ to derive the same conclusion.

We now remove the assumption we made at the beginning of the proof. Suppose that there is at least one $\sigma_{j}>N^{7}$. It follows from (4.19) and (4.20) that there are two indices $1 \leq i_{1} \neq i_{2} \leq 6$ such that $\sigma_{i_{1}}, \sigma_{i_{2}} \gtrsim N^{7}$. Then, by (2.4) and (4.24), we have

$$
\begin{aligned}
& \left|I_{1}\right| \lesssim \sum_{N \leq|n| \leq 3 N} \sum_{N_{i} \leq N ; i=1, \ldots 6} \int_{\tau}\left(\int_{\tau=\tau_{1}+\tau_{2}+\tau_{3}} \sum_{n=n_{1}+n_{2}+n_{3}}\left|\widehat{w_{N_{1}}}\right|\left|\widehat{w_{N_{2}}}\right|\left|n_{3}\right|\left|\widehat{w_{N_{3}}}\right| d \tau_{1} d \tau_{2}\right)
\end{aligned}
$$

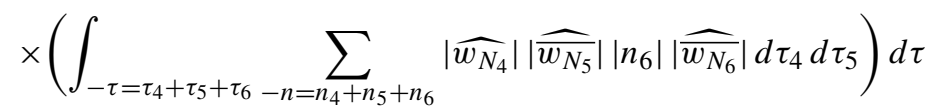

$$
\begin{aligned}
& \lesssim \sum_{N \leq|n| \leq 3 N} \sum_{N_{i} \leq N ; i=1, \ldots 6} N^{2}\left\|w_{N_{1}} w_{N_{2}} \overline{w_{N_{3}}}\right\|_{L_{x t}^{2}}\left\|w_{N_{4}} \overline{w_{N_{5}} w_{N_{6}}}\right\|_{L_{x t}^{2}} \\
& \lesssim \sum_{N \leq|n| \leq 3 N} \sum_{N_{i} \leq N ; i=1, \ldots 6} N^{-\frac{1}{3}+}\left\|w_{N_{i_{1}}}\right\|_{X^{\frac{1}{2}-, \frac{1}{2}-}}\left\|w_{N_{i_{2}}}\right\|_{X^{\frac{1}{2}-, \frac{1}{2}-}} \prod_{j \neq i_{1}, i_{2}}\left\|w_{j}\right\|_{X^{\frac{1}{2}-, \frac{1}{3}+}} \\
& \lesssim N^{-\frac{1}{3}}+\prod_{j=1}^{6}\left\|w_{j}\right\|_{X_{3}^{\frac{2}{3}-, \frac{1}{2}-}}
\end{aligned}
$$


To treat the remaining terms in (4.9) we first note that these are either higher order with no derivatives or the same order as the first but with only one derivative term. We again start by assuming that $\sigma_{j} \lesssim N^{9}$ for all $j$. Under this assumption the estimate follows from the following lemma.

Lemma 4.16 (Remaining terms). There exists $\beta>0$ such that

$$
\begin{aligned}
& \sum_{N \leq|n| \leq 3 N} \sum_{N_{i} \leq N ; i=1, \ldots 6} \int_{\tau}\left(\int_{\tau=\tau_{1}+\tau_{2}+\tau_{3}} \sum_{n=n_{1}+n_{2}+n_{3}}\left|\widehat{w_{N_{1}}}\right|\left|\widehat{w_{N_{2}}}\right|\left|\widehat{w_{N_{3}}}\right|\right) \\
& \times\left(\int_{-\tau=\tau_{4}+\tau_{5}+\tau_{6}} \sum_{-n=n_{4}+n_{5}+n_{6}}\left|\widehat{w_{N_{4}}}\right| \widehat{\mid w_{N_{5}}}|| m\left(n_{6}\right)|| \widehat{w_{N_{6}}} \mid\right) d \tau \lesssim N^{-\beta} \prod_{i=1}^{6}\left\|w_{i}\right\| X_{3}^{\frac{2}{3}-, \frac{1}{2}-}, \\
& \sum_{N \leq|n| \leq 3 N N_{i} \leq N ; i=1, \ldots 8} \int_{\tau}\left(\int_{\tau=\sum_{i=1}^{5} \tau_{i}} \sum_{n=\sum_{i=1}^{5} n_{i}}\left|\widehat{w_{N}}\right|\left|\widehat{w_{N}}\right|\left|\widehat{w_{N_{3}}}\right|\left|\widehat{w_{N_{4}}}\right|\left|\widehat{w_{N_{5}}}\right|\right) \\
& \left.\times\left(\int_{-\tau=\tau_{6}+\tau_{7}+\tau_{8}} \sum_{-n=n_{6}+n_{7}+n_{8}}\left|\widehat{w_{N_{6}}}\right|\left|\widehat{w_{N_{7}}}\right|\left|m\left(n_{8}\right)\right|\left|\widehat{w_{N_{8}}}\right|\right) d \tau \lesssim N^{-\beta} \prod_{i=1}^{8}\left\|w_{i}\right\| X_{3}^{\frac{2}{3}-, \frac{1}{2}-}\right)
\end{aligned}
$$

where the multiplier $m$ satisfies $|m(\xi)| \leq\langle\xi\rangle$.

Proof. Here we will only prove (4.39) since (4.38) is similar but simpler. Without loss of generality we can assume that $N_{1} \sim N \sim N_{8}$. Fix any $0<\sigma<1$ and consider the following cases.

Case 1. Assume that $N_{i} \lesssim N^{\sigma}, i \neq 1,8$. Then we have the support condition on $w_{N_{1}}$ and $\overline{w_{N_{8}}}$. By Plancherel, (4.39) is less than or equal to

$$
\begin{aligned}
& \sum_{N_{1}, N_{8} \sim N ; N_{i} \leq N^{\sigma}, i \neq 1,8} \int_{\mathbb{R}} \int_{\mathbb{T}} N_{8} w_{N_{1}} w_{N_{2}} \overline{w_{N_{3}}} w_{N_{4}} \overline{w_{N_{5}}} w_{N_{6}} \overline{w_{N_{7}} w_{N_{8}}} d x d t \\
& \lesssim \sum_{N_{1}, N_{8} \sim N ; N_{i} \leq N^{\sigma}, i \neq 1,8} N\left\|w_{N_{1}} w_{N_{2}} \overline{w_{N_{3}}}\right\|_{L_{x t}^{2}}\left\|w_{N_{4}} \overline{w_{N_{5}}}\right\|_{L_{x t}^{\infty}}\left\|w_{N_{6}} \overline{w_{N_{7}} w_{N_{8}}}\right\|_{L_{x t}^{2}} \\
& \lesssim \sum_{N_{1}, N_{8} \sim N ; N_{i} \leq N^{\sigma}, i \neq 1,8} N N_{1}^{-2 / 3} \max \left(N_{2}, N_{3}, N_{4}, N_{5}\right)^{1 / 6} N_{4}^{0+} N_{5}^{0+} N_{6}^{-1 / 2} N_{7}^{-1 / 2} N_{8}^{-2 / 3} \\
& \times \max \left(N_{6}, N_{7}\right)^{1 / 6}\left(\prod_{i=1}^{8}\left\|w_{N_{i}}\right\|_{X_{3}^{\frac{2}{3}-, \frac{1}{2}-}}\right) \lesssim N^{-\frac{1}{3}+\frac{\sigma}{6}+}\left(\prod_{i=1}^{8}\left\|w_{i}\right\|_{X_{3}^{\frac{2}{3}-, \frac{1}{2}-}}\right) .
\end{aligned}
$$

Case 2. Assume there exists $k \neq 1,8$ such that $N_{k}>N^{\sigma}$. Without loss of generality $k=4$. Then we bound (4.40) as follows: 


$$
\begin{gathered}
\sum_{N_{1}, N_{8} \sim N ; N_{4}>N^{\sigma}, N_{i} \leq N ; i \neq 1,4,8} N\left\|w_{N_{1}} w_{N_{4}} \overline{w_{N_{3}}}\right\|_{L_{x t}^{2}}\left\|w_{N_{2}} \overline{w_{N_{5}}}\right\|_{L_{x t}^{\infty}}\left\|w_{N_{6}} \overline{w_{N_{7}} w_{N_{8}}}\right\|_{L_{x t}^{2}} \\
\lesssim \sum_{N_{1}, N_{8} \sim N ; N_{4}>N^{\sigma}, N_{i} \leq N ; i \neq 1,4,8} N N_{1}^{-1 / 2} N_{3}^{-1 / 2} N_{4}^{-1 / 2} N_{2}^{0+} N_{5}^{0+} N_{6}^{-1 / 2} N_{7}^{-1 / 2} N_{8}^{-1 / 2} \\
\times\left(\prod_{i=1}^{8}\left\|w_{N_{i}}\right\| X_{X_{3}^{\frac{2}{3}-, \frac{1}{2}-}}\right) \lesssim N^{-\frac{\sigma}{2}+}\left(\prod_{i=1}^{8}\left\|w_{i}\right\|_{X_{3}^{\frac{2}{3}-, \frac{1}{2}-}}\right) .
\end{gathered}
$$

We now remove the assumption we made before the lemma above. Suppose that there is at least one $\sigma_{j}>N^{9}$. The term with six factors is handled just as in (4.37). To estimate the term with eight factors we first observe that as before there are at least two indices $1 \leq i_{1} \neq i_{2} \leq 8$ such that $\sigma_{i}, \sigma_{j} \gtrsim N^{9}$. Next we use Hölder's inequality to bound the left hand side of (4.39) by

$$
\sum_{N \leq|n| \leq 3 N} \sum_{N_{i} \leq N ; i=1, \ldots 8} N \prod_{i=1}^{8}\left\|w_{N_{i}}\right\|_{L_{t x}^{8}} \lesssim \sum_{N \leq|n| \leq 3 N} \sum_{N_{i} \leq N ; i=1, \ldots 8} N \prod_{i=1}^{8}\left\|w_{N_{i}}\right\|_{X_{3}^{\frac{13}{24}+, \frac{3}{8}}+}
$$

by (4.25). Using $\sigma_{i_{1}}, \sigma_{i_{2}}>N^{9}$ we conclude that the above is

$$
\begin{aligned}
& \lesssim \sum_{N \leq|n| \leq 3 N} \sum_{N_{i} \leq N ; i=1, \ldots 8} N^{-\frac{1}{8}+}\left\|w_{N_{i_{1}}}\right\|_{X_{3}^{\frac{13}{24}+, \frac{1}{2}-}}\left\|w_{N_{i_{2}}}\right\|_{X_{3}^{\frac{13}{24}+, \frac{1}{2}-}} \prod_{i \neq i_{1}, i_{2}}\left\|w_{N_{i}}\right\|_{X_{3}^{\frac{13}{24}+, \frac{3}{8}+}} \\
& \lesssim N^{-\frac{1}{8}+} \prod_{i=1}^{8}\left\|w_{i}\right\|_{X_{3}^{\frac{2}{3}-, \frac{1}{2}-} \cdot}
\end{aligned}
$$

\section{Construction of weighted Wiener measures}

In this section we construct weighted Wiener measures and associated probability spaces on which we establish well-posedness. To construct these measures we make use of the conserved quantities $\mathcal{E}(v)$ (given in (2.13)) and the $L^{2}$-norm. As a motivation we recall a well known fact in finite-dimensional spaces. Suppose we have a well-posed ODE $y_{t}=F(y)$, where $F$ is a divergence-free vector field. Assume $G(y)$ is a constant of motion such that for reasonable $f, f(G(y)) \in L^{1}(d y)$. Then by Liouville's Theorem, $d \mu(y)=Z^{-1} f(G(y)) d y$ is, for a suitable normalization constant $Z$, an invariant probability measure for the flow map for the ODE.

To construct measures on infinite-dimensional spaces we will consider conserved quantities of the form $\exp \left(-\frac{\beta}{2} \mathcal{E}(v)\right)$. But there is a priori little hope of constructing a finite measure using this quantity since (a) the nonlinear part of $\mathcal{E}(v)$ is not bounded below and (b) the linear part is only nonnegative but not positive definite. To resolve this we use the conservation of $L^{2}$-norm and consider instead the conserved quantity

$$
\chi\left\{\|v\|_{L^{2}} \leq B\right\} e^{-\frac{\beta}{2} \mathcal{N}(v)} e^{-\frac{\beta}{2} \int\left(|v|^{2}+\left|v_{x}\right|^{2}\right) d x}
$$


where $\mathcal{N}(v)$ is the nonlinear part of the energy, i.e.

$$
\begin{aligned}
\mathcal{N}(v)= & -\frac{1}{2} \operatorname{Im} \int_{\mathbb{T}} v^{2} \overline{v v}_{x} d x-\frac{1}{4 \pi}\left(\int_{\mathbb{T}}|v|^{2} d x\right)\left(\int_{\mathbb{T}}|v|^{4} d x\right) \\
& +\frac{1}{\pi}\left(\int_{\mathbb{T}}|v|^{2} d x\right)\left(\operatorname{Im} \int_{\mathbb{T}} v \bar{v}_{x} d x\right)+\frac{1}{4 \pi^{2}}\left(\int_{\mathbb{T}}|v|^{2} d x\right)^{3},
\end{aligned}
$$

and $B$ is a (suitably small) constant.

By analogy with the finite-dimensional case we would like to construct the measure (with $v(x)=u(x)+i w(x))$

$$
\text { “ } d \mu_{\beta}=Z^{-1} \chi\left\{\|v\|_{\left.L^{2} \leq B\right\}} e^{-\frac{\beta}{2} \mathcal{N}(v)} e^{-\frac{\beta}{2} \int\left(|v|^{2}+\left|v_{x}\right|^{2}\right) d x} \prod_{x \in \mathbb{T}} d u(x) d w(x) ” .\right.
$$

This is a purely formal, although suggestive, expression since it is impossible to define the Lebesgue measure on an infinite-dimensional space as a countably additive measure. Moreover, it will turn out that $\int\left|u_{x}\right|^{2}=\infty, \mu$-almost surely.

One uses instead a Gaussian measure as reference measure and the measure $\mu$ is constructed in two steps. First one constructs a Gaussian measure $\rho$ as the limit of the finite-dimensional measures on $\mathbb{R}^{4 N+2}$ given by

$$
d \rho_{N}=Z_{0, N}^{-1} \exp \left(-\frac{\beta}{2} \sum_{|n| \leq N}\left(1+|n|^{2}\right)\left|\widehat{v}_{n}\right|^{2}\right) \prod_{|n| \leq N} d a_{n} d b_{n}
$$

where $\widehat{v}_{n}=a_{n}+i b_{n}$. The construction of such Gaussian measures is a classical subject (see e.g. Gross [20] and Kuo [29]). For our purpose we will need to realize this measure as a measure supported on a suitable Banach space. Once this measure $\rho$ has been constructed one constructs the measure $\mu$ as a measure which is absolutely continuous with respect to $\rho$ and whose Radon-Nikodym derivative is

$$
\frac{d \mu}{d \rho}=\tilde{Z}^{-1} \chi_{\left\{\|v\|_{L}^{2} \leq B\right\}} e^{-\frac{\beta}{2} \mathcal{N}(v)} .
$$

For this measure to be normalizable it turns out that one needs $B$ to be sufficiently small. Also the constant $\beta$ in the measure does not play any role in the analysis (although the cutoff $B$ depends on $\beta$ ) and thus in the sequel we will set $\beta=1$. But note that the measures for different $\beta$ are all invariant and they are all mutually singular [20, 29].

First let us recall some facts on Gaussian measures in Hilbert spaces and Banach spaces. For details see Zhidkov [48], Gross [20] and Kuo [29]. Let $n \in \mathbb{N}$ and $\mathcal{T}$ be a symmetric positive $n \times n$ matrix with real entries. The Borel measure $\rho$ in $\mathbb{R}^{n}$ given by

$$
d \rho(x)=\frac{1}{\sqrt{(2 \pi)^{n} \operatorname{det}(\mathcal{T})}} \exp \left(-\frac{1}{2}\left\langle\mathcal{T}^{-1} x, x\right\rangle_{\mathbb{R}^{n}}\right) d x
$$

is called a (nondegenerate centered) Gaussian measure in $\mathbb{R}^{n}$. Note that $\rho\left(\mathbb{R}^{n}\right)=1$.

Now, we consider the analogous definition of the infinite-dimensional (centered) Gaussian measures. Let $H$ be a real separable Hilbert space and $\mathcal{T}: H \rightarrow H$ be a 
linear positive self-adjoint operator (generally unbounded) with eigenvalues $\left\{\lambda_{n}\right\}_{n \in \mathbb{N}}$ and the corresponding eigenvectors $\left\{e_{n}\right\}_{n \in \mathbb{N}}$ forming an orthonormal basis of $H$. We call a set $M \subset H$ cylindrical if there exists an integer $n \geq 1$ and a Borel set $F \subset \mathbb{R}^{n}$ such that

$$
M=\left\{x \in H:\left(\left\langle x, e_{1}\right\rangle_{H}, \ldots,\left\langle x, e_{n}\right\rangle_{H}\right) \in F\right\} .
$$

Given the operator $\mathcal{T}$, we denote by $\mathcal{A}$ the set of all cylindrical subsets of $H$; one can easily verify that $\mathcal{A}$ is a field. The centered Gaussian measure in $H$ with correlation operator $\mathcal{T}$ is defined as the additive (but not countably additive in general) measure $\rho$ defined on the field $\mathcal{A}$ via

$$
\rho(M)=(2 \pi)^{-n / 2} \prod_{j=1}^{n} \lambda_{j}^{-1 / 2} \int_{F} e^{-\frac{1}{2} \sum_{j=1}^{n} \lambda_{j}^{-1} x_{j}^{2}} d x_{1} \cdots d x_{n} \quad \text { for } M \in \mathcal{A} \text { as in (5.3). }
$$

The following proposition tells us when this Gaussian measure $\rho$ is countably additive.

Proposition 5.1. The Gaussian measure $\rho$ defined in (5.4) is countably additive on the field $\mathcal{A}$ if and only if $\mathcal{T}$ is an operator of trace class, i.e., $\sum_{n=1}^{\infty} \lambda_{n}<\infty$. If the latter holds, then the minimal $\sigma$-field $\mathcal{M}$ containing the field $\mathcal{A}$ of all cylindrical sets is the Borel $\sigma$-field on $H$.

Consider a sequence of finite-dimensional Gaussian measures $\left\{\rho_{n}\right\}_{n \in \mathbb{N}}$ defined as follows. For fixed $n \in \mathbb{N}$, let $\mathcal{M}_{n}$ be the set of all cylindrical sets in $H$ of the form (5.3) with this fixed $n$ and arbitrary Borel sets $F \subset \mathbb{R}^{n}$. Clearly, $\mathcal{M}_{n}$ is a $\sigma$-field, and setting

$$
\rho_{n}(M)=(2 \pi)^{-n / 2} \prod_{j=1}^{n} \lambda_{j}^{-1 / 2} \int_{F} e^{-\frac{1}{2} \sum_{j=1}^{n} \lambda_{j}^{-1} x_{j}^{2}} d x_{1} \cdots d x_{n}
$$

for $M \in \mathcal{M}_{n}$, we obtain a countably additive measure $\rho_{n}$ defined on $\mathcal{M}_{n}$. Then, one can extend the measure $\rho_{n}$ onto the whole Borel $\sigma$-field $\mathcal{M}$ of $H$ by setting $\rho_{n}(A):=$ $\rho_{n}\left(A \cap \operatorname{span}\left\{e_{1}, \ldots, e_{n}\right\}\right)$ for $A \in \mathcal{M}{ }^{6}$ Then we have

Proposition 5.2. Let $\rho$ in (5.4) be countably additive. Then $\left\{\rho_{n}\right\}_{n \in \mathbb{N}}$ constructed above converges weakly to $\rho$ as $n \rightarrow \infty$.

For our problem we consider the Gaussian measure $\rho$ which is the weak limit of the finite-dimensional Gaussian measures

$$
d \rho_{N}=Z_{0, N}^{-1} \exp \left(-\frac{1}{2} \sum_{|n| \leq N}\left(1+|n|^{2}\right)\left|\widehat{v}_{n}\right|^{2}\right) \prod_{|n| \leq N} d a_{n} d b_{n}
$$

Let $J_{s}:=(1-\Delta)^{s-1}$. Then we have

$$
\sum_{n}\left(1+|n|^{2}\right)\left|\widehat{v}_{n}\right|^{2}=\langle v, v\rangle_{H^{1}}=\left\langle J_{s}^{-1} v, v\right\rangle_{H_{s}} .
$$

\footnotetext{
6 Note a slight abuse of notation. We use $\rho_{n}$ to denote a Gaussian measure on $\operatorname{span}\left\{e_{1}, \ldots, e_{n}\right\}$ as well as its extension on $H$. A similar comment applies in the following.
} 
The operator $J_{S}: H_{S} \rightarrow H_{S}$ has the set of eigenvalues $\left\{\left(1+|n|^{2}\right)^{s-1}\right\}_{n \in \mathbb{Z}}$ and the corresponding eigenvectors $\left\{\left(1+|n|^{2}\right)^{-s / 2} e^{i n x}\right\}_{n \in \mathbb{Z}}$ form an orthonormal basis of $H^{s}$. Since $J_{S}$ is of trace class if and only if $s<1 / 2$, by Proposition 5.1, $\rho$ is a countably additive measure on $H^{s}$ for any $s<1 / 2$ (but not for $s \geq 1 / 2$ ).

Unfortunately, (2.6) is locally well-posed in $H^{s}(\mathbb{T})$ only for $s \geq 1 / 2$ [26]. Instead, we propose to work in the Fourier-Lebesgue space $\mathcal{F} L^{s, r}(\mathbb{T})$ defined in (2.2) in view of the local well-posedness result by Grünrock-Herr [22]. Since $\mathcal{F} L^{s, r}$ is not a Hilbert space, we need to construct $\rho$ as a measure supported on a Banach space.

\subsection{General Banach space setting}

Let us recall the basic theory of abstract Wiener spaces [29]. Given a real separable Hilbert space $H$ with norm $\|\cdot\|$, let $\mathcal{F}$ denote the set of finite-dimensional orthogonal projections $\mathbb{P}$ of $H$. Then define a cylinder set $E$ by $E=\{x \in H: \mathbb{P} x \in F\}$ where $\mathbb{P} \in \mathcal{F}$ and $F$ is a Borel subset of $\mathbb{P} H$, and let $\mathcal{R}$ denote the collection of such cylinder sets. Note that $\mathcal{R}$ is a field but not a $\sigma$-field. The Gaussian measure $\rho$ on $H$ is defined by

$$
\rho(E)=(2 \pi)^{-n / 2} \int_{F} e^{-\|x\|^{2} / 2} d x
$$

for $E \in \mathcal{R}$, where $n=\operatorname{dim} \mathbb{P} H$ and $d x$ is the Lebesgue measure on $\mathbb{P} H$. It is known that $\rho$ is finitely additive but not countably additive in $\mathcal{R}$.

Definition 5.3 (Gross [20]). A seminorm $\||\cdot|||$ in $H$ is called measurable if for every $\varepsilon>0$, there exists $\mathbb{P}_{\varepsilon} \in \mathcal{F}$ such that

$$
\rho(\||\mathbb{P} x|\|>\varepsilon)<\varepsilon
$$

for $\mathbb{P} \in \mathcal{F}$ orthogonal to $\mathbb{P}_{\varepsilon}$.

Any measurable seminorm is weaker than the norm of $H$, and $H$ is not complete with respect to $\||\cdot|\|$ unless $H$ is finite-dimensional. Let $\mathcal{B}$ be the completion of $H$ with respect to $\||\cdot|\|$ and denote by $i$ the inclusion map of $H$ into $\mathcal{B}$. The triple $(i, H, \mathcal{B})$ is called an abstract Wiener space.

Now, regarding $y \in \mathcal{B}^{*}$ as an element of $H^{*} \equiv H$ by restriction, we embed $\mathcal{B}^{*}$ in $H$. Define the extension of $\rho$ onto $\mathcal{B}$ (still denoted by $\rho$ ) as follows. For a Borel set $F \subset \mathbb{R}^{n}$, set

$\rho\left(\left\{x \in \mathcal{B}:\left(\left(x, y_{1}\right), \ldots,\left(x, y_{n}\right)\right) \in F\right\}\right):=\rho\left(\left\{x \in H:\left(\left\langle x, y_{1}\right\rangle_{H}, \ldots,\left\langle x, y_{n}\right\rangle_{H}\right) \in F\right\}\right)$,

where $y_{j}$ 's are in $\mathcal{B}^{*}$ and $(\cdot, \cdot)$ denotes the natural pairing between $\mathcal{B}$ and $\mathcal{B}^{*}$. Let $\mathcal{R}_{\mathcal{B}}$ denote the collection of cylinder sets $\left\{x \in \mathcal{B}:\left(\left(x, y_{1}\right), \ldots,\left(x, y_{n}\right)\right) \in F\right\}$ in $\mathcal{B}$.

Proposition 5.4 (Gross [20]). $\rho$ is countably additive on the $\sigma$-field generated by $\mathcal{R}_{\mathcal{B}}$.

\subsection{Back to our setting}

In the present context, we will let $H=H^{1}(\mathbb{T})$ and $\mathcal{B}=\mathcal{F} L^{s, r}(\mathbb{T})$ with $2 \leq r<\infty$ and $(s-1) r<-1$. First we prove the following result. 
Proposition 5.5. Let $2 \leq r<\infty$ and assume $(s-1) r<-1$. Then the seminorm $\|\cdot\|_{\mathcal{F}} L^{s, r}$ is measurable. Moreover, we have the following exponential tail estimate: there exist $C>0$ and $c>0$ (which both depend on $(s, r)$ ) such that, for $K>0$,

$$
\rho\left(\|v\|_{\mathcal{F} L^{s, r}}>K\right) \leq C e^{-c K^{2}} .
$$

This shows that $(i, H, \mathcal{B})=\left(i, H^{1}, \mathcal{F} L^{s, r}\right)(2 \leq r<\infty)$ is an abstract Wiener space if $(s-1) r<-1$ and thus the Wiener measure $\rho$ can be realized as a countably additive measure supported on $\mathcal{F} L^{s, r}$ for $(s-1) r<-1$. This is hardly surprising since this is equivalent to $\sigma \equiv s+1 / r-1 / 2<1 / 2$, and $\mathcal{F} L^{s, r}$ scale as $H^{\sigma}$.

The second part of Proposition 5.5 is a consequence of Fernique's theorem [19] (cf. Theorem 3.1 of Chapter III in [29]).

Remark 5.6. Proposition 5.5 was essentially proved in [35] in the context of white noise for the $\mathrm{KdV}$ equation. We include here a proof in our DNLS context for completeness. ${ }^{7}$

It is useful to note that the measure $\rho_{N}$ given in (5.5) can be regarded as the induced probability measure on $\mathbb{C}^{2 N+1} \cong \mathbb{R}^{4 N+2}$ under the map

$$
\omega \mapsto\left\{g_{n} / \sqrt{1+|n|^{2}}\right\}_{|n| \leq N},
$$

where $g_{n}(\omega),|n| \leq N$, are independent standard complex Gaussian random variables on a probability space $(\Omega, \mathcal{F}, P)$ (i.e. $\widehat{v}_{n}=g_{n} / \sqrt{1+|n|^{2}}$ ). In a similar manner, we can view $\rho$ as the induced probability measure under the map $\omega \mapsto\left\{g_{n} / \sqrt{1+|n|^{2}}\right\}_{n \in \mathbb{Z}}$, where $g_{n}(\omega)$ are independent standard complex Gaussian random variables.

For the proof of Proposition 5.5, we first recall the following result.

Lemma 5.7 ([36, Lemma 4.7]). Let $\left\{g_{n}\right\}$ be a sequence of independent standard complex-valued Gaussian random variables. Then, for $M$ dyadic and $\delta<1 / 2$, we have

$$
\lim _{M \rightarrow \infty} M^{2 \delta} \frac{\max _{|n| \sim M}\left|g_{n}\right|^{2}}{\sum_{|n| \sim M}\left|g_{n}\right|^{2}}=0 \quad \text { a.s. }
$$

Proof of Proposition 5.5. Let $2 \leq r<\infty$ and $(s-1) r<-1$. In view of Definition 5.3, it suffices to show that for given $\varepsilon>0$, there exists a large $M_{0}$ such that

$$
\rho\left(\left\|P_{M_{0}}^{\perp} v\right\|_{\mathcal{F} L^{s, r}}>\varepsilon\right)<\varepsilon,
$$

where $P_{M_{0}}^{\perp}$ is the projection onto the frequencies $|n|>M_{0}$. Note that if $\mathbb{P}$ is a finitedimensional projection such that $\mathbb{P} \perp P_{M_{0}}$ then $\|\mathbb{P} v\|_{\mathcal{F} L^{s, r}} \leq\left\|P_{M_{0}}^{\perp} v\right\|_{\mathcal{F} L^{s, r} \text {. }}$.

In view of (5.7), we assume that $v$ is of the form

$$
v(x)=\sum_{n} \frac{g_{n}}{\sqrt{1+|n|^{2}}} e^{i n x},
$$

where $\left\{g_{n}\right\}$ is as in (5.7).

7 Proposition 5.5 also holds for $r<2$ and $(s-1) r<-1$, albeit with a different proof (see [1] for details). For our purposes $2 \leq r<\infty$ suffices and so we restrict ourselves to that case. 
Let $\delta<1 / 2$ to be chosen later. Then, by Lemma 5.7 and Egoroff's theorem, there exists a set $E$ such that $\rho\left(E^{c}\right)<\frac{1}{2} \varepsilon$ and the convergence in Lemma 5.7 is uniform on $E$, i.e. we can choose dyadic $M_{0}$ large enough such that

$$
\frac{\left\|\left\{g_{n}(\omega)\right\}_{|n| \sim M}\right\|_{L_{n}^{\infty}}}{\left\|\left\{g_{n}(\omega)\right\}_{|n| \sim M}\right\|_{L_{n}^{2}}} \leq M^{-\delta}
$$

for all $\omega \in E$ and dyadic $M>M_{0}$. In the following, we will work only on $E$ and drop ' $\cap E$ ' for notational simplicity. However, it should be understood that all the events are under the intersection with $E$ so that (5.9) holds.

Let $\left\{\sigma_{j}\right\}_{j \geq 1}$ be a sequence of positive numbers such that $\sum \sigma_{j}=1$, and let $M_{j}=$ $M_{0} 2^{j}$ dyadic. Note that $\sigma_{j}=C 2^{-\lambda j}=C M_{0}^{\lambda} M_{j}^{-\lambda}$ for some small $\lambda>0$ (to be determined later). Then, from (5.8), we have

$$
\rho\left(\left\|P_{M_{0}}^{\perp} v(\omega)\right\| \mathcal{F} L^{s, r}>\varepsilon\right) \leq \sum_{j=1}^{\infty} \rho\left(\left\|\left\{\langle n\rangle^{s-1} g_{n}(\omega)\right\}_{|n| \sim M_{j}}\right\|_{L_{n}^{r}>\sigma_{j} \varepsilon}\right) .
$$

By interpolation and (5.9),

$$
\begin{aligned}
& \left\|\left\{\langle n\rangle^{s-1} g_{n}\right\}_{|n| \sim M_{j}}\right\|_{L_{n}^{r}} \\
& \quad \sim M_{j}^{s-1}\left\|\left\{g_{n}\right\}_{|n| \sim M_{j}}\right\|_{L_{n}^{r}} \leq M_{j}^{s-1}\left\|\left\{g_{n}\right\}_{|n| \sim M_{j}}\right\|_{L_{n}^{2}}^{2 / r}\left\|\left\{g_{n}\right\}_{|n| \sim M_{j}}\right\|_{L_{n}^{\infty}}^{(r-2) / r} \\
& \quad \leq M_{j}^{s-1}\left\|\left\{g_{n}\right\}_{|n| \sim M}\right\|_{L_{n}^{2}}\left(\frac{\left\|\left\{g_{n}\right\}_{|n| \sim M_{j}}\right\|_{L_{n}^{\infty}}}{\left\|\left\{g_{n}\right\}_{|n| \sim M_{j}}\right\|_{L_{n}^{2}}}\right)^{(r-2) / r} \leq M_{j}^{s-1-\delta(r-2) / r}\left\|\left\{g_{n}\right\}_{|n| \sim M_{j}}\right\|_{L_{n}^{2}} .
\end{aligned}
$$

Thus, if $\left\|\left\{\langle n\rangle^{s-1} g_{n}\right\}_{|n| \sim M_{j}}\right\|_{L_{n}^{r}}>\sigma_{j} \varepsilon$, then $\left\|\left\{g_{n}\right\}_{|n| \sim M_{j}}\right\|_{L_{n}^{2}} \gtrsim R_{j}$ where $R_{j}:=\sigma_{j} \varepsilon M_{j}^{\alpha}$ with $\alpha:=-s+1+\delta(r-2) / r$. With $r=2+\theta$, we have

$$
\alpha=\frac{-(s-1) r+\delta \theta}{2+\theta}>\frac{1}{2}
$$

by taking $\delta$ sufficiently close to $1 / 2$ since $-(s-1) r>1$. Then, by taking $\lambda>0$ sufficiently small, $R_{j}=\sigma_{j} \varepsilon M_{j}^{\alpha}=C \varepsilon M_{0}^{\lambda} M_{j}^{\alpha-\lambda} \gtrsim C \varepsilon M_{0}^{\lambda} M_{j}^{(1 / 2)+}$. By a direct computation in polar coordinates, we have

$$
\rho\left(\left\|\left\{g_{n}\right\}_{|n| \sim M_{j}}\right\|_{L_{n}^{2}} \gtrsim R_{j}\right) \sim \int_{B^{c}\left(0, R_{j}\right)} e^{-\frac{1}{2}\left|g_{n}\right|^{2}} \prod_{|n| \sim M_{j}} d g_{n} \lesssim \int_{R_{j}}^{\infty} e^{-\frac{1}{2} s^{2}} s^{2 \#\left\{|n| \sim M_{j}\right\}-1} d s .
$$

Note that, in the inequality, we have dropped the implicit constant $\sigma\left(S^{2 \#\left\{|n| \sim M_{j}\right\}-1}\right)$, a surface measure of the $2 \#\left\{|n| \sim M_{j}\right\}-1$-dimensional unit sphere, since $\sigma\left(S^{n}\right)=$ $2 \pi^{n / 2} / \Gamma(n / 2) \lesssim 1$. By the change of variable $t=M_{j}^{-1 / 2} s$, we have $s^{2 \#\left\{|n| \sim M_{j}\right\}-2} \lesssim$ $s^{4 M_{j}} \sim M_{j}^{2 M_{j}} t^{4 M_{j}}$. Since $t>M_{j}^{-1 / 2} R_{j}=C \varepsilon M_{0}^{\lambda} M_{j}^{0+}$, we have $M_{j}^{2 M_{j}}=e^{2 M_{j} \ln M_{j}}<$ 
$e^{\frac{1}{8} M_{j} t^{2}}$ and $t^{4 M_{j}}<e^{\frac{1}{8} M_{j} t^{2}}$ for $M_{0}$ sufficiently large. Thus, $s^{2 \#\left\{|n| \sim M_{j}\right\}-2}<e^{\frac{1}{4} M_{j} t^{2}}=e^{\frac{1}{4} s^{2}}$ for $s>R_{j}$. Hence,

$$
\rho\left(\left\|\left\{g_{n}\right\}_{|n| \sim M_{j}}\right\|_{L_{n}^{2}} \gtrsim R_{j}\right) \leq C \int_{R_{j}}^{\infty} e^{-\frac{1}{4} s^{2}} s d s \leq e^{-c R_{j}^{2}}=e^{-c C^{2} M_{0}^{2 \lambda} M_{j}^{1+} \varepsilon^{2}} .
$$

From (5.10) and (5.11), we have

$$
\rho\left(\left\|P_{M_{0}}^{\perp} v\right\|_{\mathcal{F} L^{s, r}}>\varepsilon\right) \leq \sum_{j=1}^{\infty} e^{-c C^{2} M_{0}^{1+2 \lambda+}\left(2^{j}\right)^{1+} \varepsilon^{2}} \leq \frac{1}{2} \varepsilon
$$

by choosing $M_{0}$ sufficiently large as long as $(s-1) r<-1$. Hence, the seminorm $\|\cdot\|_{\mathcal{F} L^{s, r}}$ is measurable for $(s-1) r<-1$.

The tail estimate (5.6) is a direct consequence of Fernique's theorem [29, Theorem 3.1].

To construct the weighted Wiener measure $\mu$ let us define

$$
R(v):=\chi_{\left\{\|v\|_{L^{2}} \leq B\right\}} e^{-\frac{1}{2} \mathcal{N}(v)}, \quad R_{N}(v):=R\left(v^{N}\right),
$$

where $\mathcal{N}(v)$ is the nonlinear part of the energy defined in (5.1) and at this stage and for the remainder of this section $v^{N}=P_{N}(v)$ for some generic function $v$. In the next section $v^{N}$ will denote the solution to the FGDNLS (3.1) as in Section 3. We write

$$
\mathcal{N}_{N}(v):=\mathcal{N}\left(v^{N}\right)=F_{N}(v)+G_{N}(v)+K_{N}(v)
$$

where

$$
\begin{aligned}
F_{N}(v) & =-\frac{1}{2} \operatorname{Im} \int_{\mathbb{T}}\left(v^{N}\right)^{2} \overline{v^{N}} \overline{v_{x}^{N}} d x \\
G_{N}(v) & =-\frac{1}{4 \pi}\left(\int_{\mathbb{T}}\left|v^{N}\right|^{2} d x\right)\left(\int_{\mathbb{T}}\left|v^{N}\right|^{4} d x\right), \\
K_{N}(v) & =\frac{1}{\pi}\left(\int_{\mathbb{T}}\left|v^{N}\right|^{2} d x\right)\left(\operatorname{Im} \int_{\mathbb{T}} v^{N} \overline{v_{x}^{N}} d x\right)+\frac{1}{4 \pi^{2}}\left(\int_{\mathbb{T}}\left|v^{N}\right|^{2} d x\right)^{3} .
\end{aligned}
$$

We will construct the measure

$$
d \mu=Z^{-1} R(v) d \rho
$$

for sufficiently small $B$, as the weak limit of the finite-dimensional weighted Wiener measures $\mu_{N}$ on $\mathbb{R}^{4 N+2}$ given by

$$
d \mu_{N}=Z_{N}^{-1} R_{N}(v) d \rho_{N}=Z_{N}^{-1} \chi_{\left\{\left\|v^{N}\right\|_{L^{2}} \leq B\right\}} e^{-\frac{1}{2} \mathcal{N}\left(v^{N}\right)} d \rho_{N}
$$

for a suitable normalization $Z_{N}$. 
Lemma 5.8. (a) The sequence $F_{N}$ converges in $L^{2}(d \rho)$ to

$$
F(v)=-\frac{1}{2} \operatorname{Im} \int_{\mathbb{T}} v^{2} \bar{v} \overline{v_{x}} d x .
$$

Moreover, for $\alpha<3 / 4$, there are $C, \delta>0$ such that for all $M \geq N \geq 1$ and $\lambda>0$,

$$
\rho\left(\left|F_{M}(v)-F_{N}(v)\right|>\lambda\right) \leq C e^{-\delta N^{\alpha} \lambda^{1 / 2}}
$$

(b) Let $p \in[2, \infty)$. Then there exist $\alpha, C$ such that for all $M \geq N \geq 1$ and $\lambda>0$,

$$
\begin{gathered}
\rho\left(\left\|P_{N} v\right\|_{L^{p}(\mathbb{T})}>\lambda\right)<C e^{-c \lambda^{2}}, \\
\rho\left(\left\|P_{M} v-P_{N} v\right\|_{L^{p}(\mathbb{T})}>\lambda\right)<C e^{-c N^{2 \alpha} \lambda^{2}} .
\end{gathered}
$$

Proof. Part (a) was proved by Thomann and Tzvetkov in [42, Proposition 3.1] using Proposition 5.10 below. Note that their proof only uses the fact that $v$ is in the support of the measure and is independent of the function space $v$ is in.

To prove (b) we first note that for any $2 \leq p<\infty$ and $N \leq M$,

$$
\begin{gathered}
\left\|P_{N} v\right\|_{L^{p}(\mathbb{T})} \leq C\left\|P_{N} v\right\|_{\mathcal{F} L^{\frac{2}{3}-, 3}(\mathbb{T})}, \\
\left\|P_{N} v-P_{M} v\right\|_{L^{p}(\mathbb{T})} \leq C \frac{1}{N^{\alpha}}\left\|P_{M} v\right\|_{\mathcal{F} L^{\frac{2}{3}-, 3}(\mathbb{T})},
\end{gathered}
$$

where $\alpha=(1 / p)-$. Then use (5.16) and (5.17) in conjunction with (5.6) to conclude the proof.

Lemma 5.9. $K_{N}(v)$ is Cauchy in measure, i.e. for every $\gamma>0$ and $N \leq M$,

$$
\lim _{N, M \rightarrow \infty} \rho\left(\left|K_{M}(v)-K_{N}(v)\right|>2 \gamma\right)=0,
$$

and hence $K_{N}$ converges in measure to

$$
K(v)=\frac{1}{\pi}\left(\int_{\mathbb{T}}|v|^{2} d x\right)\left(\operatorname{Im} \int_{\mathbb{T}} v \overline{v_{x}} d x\right)+\frac{1}{4 \pi^{2}}\left(\int_{\mathbb{T}}|v|^{2} d x\right)^{3} .
$$

Before the proof we need the following Proposition 5.10 (see Thomann and Tzevtkov [42] for a proof) and Lemma 5.11 which we prove below.

Proposition 5.10. Let $d \geq 1$ and $c\left(n_{1}, \ldots, n_{k}\right) \in \mathbb{C}$. Let $\left\{g_{n}\right\}_{1 \leq n \leq d} \in \mathcal{N}_{\mathbb{C}}(0,1)$ be complex $L^{2}$ normalized independent Gaussians. For $k \geq 1$ set $A(k, d):=\left\{\left(n_{1}, \ldots, n_{k}\right) \in\right.$ $\left.\{1, \ldots, d\}^{k}: n_{1} \leq \cdots \leq n_{k}\right\}$ and

$$
S_{k}(\omega)=\sum_{A(k, d)} c\left(n_{1}, \ldots, n_{k}\right) g_{n_{1}}(\omega) \ldots g_{n_{k}}(\omega) .
$$

Then for all $d \geq 1$ and $p \geq 2$,

$$
\left\|S_{k}\right\|_{L^{p}(\Omega)} \leq \sqrt{k+1}(p-1)^{k / 2}\left\|S_{k}\right\|_{L^{2}(\Omega)} .
$$

Let $X_{N}(v)=\int_{\mathbb{T}} v^{N} \overline{v_{x}^{N}}$. 
Lemma 5.11. For any $N \leq M$ and $\varepsilon>0$ we have

$$
\begin{aligned}
\left|X_{N}(v)\right| & \lesssim N^{2 \varepsilon}\left\|v^{N}\right\|_{\mathcal{F} L^{2 / 3-\varepsilon, 3}}^{2}, \\
\left\|X_{M}(v)-X_{N}(v)\right\|_{L^{4}} & \lesssim \frac{1}{N^{1 / 2}}, \\
\left\|X_{M}(v)-X_{N}(v)\right\|_{L^{q}} & \lesssim c(q-1) \frac{1}{N^{1 / 2}} \quad \text { for any } q \geq 2 .
\end{aligned}
$$

Proof. To prove (5.19) we use Plancherel and Hölder's inequality to obtain

$$
\begin{aligned}
\left|X_{N}(v)\right| & \leq \sum_{|n| \leq N}|n|\left|\widehat{v^{N}}(n)\right|^{2} \\
& \leq\left(\sum_{|n| \leq N}|n|^{-1+6 \varepsilon}\right)^{1 / 3}\left(\sum_{|n| \leq N}\left(|n|^{2 / 3-\varepsilon}\left|\widehat{v^{N}}(n)\right|\right)^{3}\right)^{2 / 3} \leq N^{2 \varepsilon}\left\|v^{N}\right\|_{\mathcal{F} L^{2 / 3-\varepsilon, 3}}^{2} .
\end{aligned}
$$

To prove (5.20) we start by recalling that $v^{N}(\omega, x):=\sum_{|n| \leq N} \frac{g_{n}(\omega)}{\langle n\rangle} e^{i n x}$. Then by Plancherel,

$$
X_{N}(v)=-i \sum_{|n| \leq N} n \frac{\left|g_{n}(\omega)\right|^{2}}{\langle n\rangle^{2}} \quad \text { and } \quad X_{M}(v)-X_{N}(v)=-i \sum_{N \leq|n|<M} n \frac{\left|g_{n}(\omega)\right|^{2}}{\langle n\rangle^{2}},
$$

and

$$
\left|X_{M}(v)-X_{N}(v)\right|^{2}=\sum_{N \leq\left|n_{1}\right|,\left|n_{2}\right|<M} n_{1} n_{2} \frac{\left|g_{n_{1}}(\omega)\right|^{2}\left|g_{n_{2}}(\omega)\right|^{2}}{\left\langle n_{1}\right\rangle^{2}\left\langle n_{2}\right\rangle^{2}}=: Y_{N, M}^{1}+Y_{N, M}^{2}+Y_{N, M}^{3},
$$

where

$$
\begin{aligned}
Y_{N, M}^{1} & :=\sum_{N \leq\left|n_{1}\right|,\left|n_{2}\right|<M} n_{1} n_{2} \frac{\left(\left|g_{n_{1}}(\omega)\right|^{2}-1\right)\left(\left|g_{n_{2}}(\omega)\right|^{2}-1\right)}{\left\langle n_{1}\right\rangle^{2}\left\langle n_{2}\right\rangle^{2}}, \\
Y_{N, M}^{2} & :=\sum_{N \leq\left|n_{1}\right|,\left|n_{2}\right|<M} n_{1} n_{2} \frac{\left(\left|g_{n_{1}}(\omega)\right|^{2}-1\right)+\left(\left|g_{n_{2}}(\omega)\right|^{2}-1\right)}{\left\langle n_{1}\right\rangle^{2}\left\langle n_{2}\right\rangle^{2}}, \\
Y_{N, M}^{3} & :=\sum_{N \leq\left|n_{1}\right|,\left|n_{2}\right|<M} \frac{n_{1} n_{2}}{\left\langle n_{1}\right\rangle^{2}\left\langle n_{2}\right\rangle^{2}} .
\end{aligned}
$$

By symmetry $Y_{N, M}^{3}=0$. We now observe that

$$
\left\|X_{M}(v)-X_{N}(v)\right\|_{L^{4}}^{4} \lesssim\left\|Y_{N, M}^{1}\right\|_{L^{2}}^{2}+\left\|Y_{N, M}^{2}\right\|_{L^{2}}^{2} .
$$

We now proceed as in [42]. Set $G_{n}(\omega):=\left|g_{n}(\omega)\right|^{2}-1$ and note that by the independence of $g_{n}(\omega)$ (cf. (5.7)),

$$
\mathbb{E}\left[G_{n}(\omega) G_{m}(\omega)\right]=0 \quad \text { for } n \neq m .
$$


Since

$$
\left|Y_{N, M}^{1}\right|^{2}=\sum_{N \leq\left|n_{1}\right|,\left|n_{2}\right|,\left|n_{3}\right|,\left|n_{4}\right|<M} n_{1} n_{2} n_{3} n_{4} \frac{G_{n_{1}} G_{n_{2}} G_{n_{3}} G_{n_{4}}}{\left\langle n_{1}\right\rangle^{2}\left\langle n_{2}\right\rangle^{2}\left\langle n_{3}\right\rangle^{2}\left\langle n_{4}\right\rangle^{2}}
$$

We compute $\mathbb{E}\left[\left|Y_{N, M}^{1}\right|^{2}\right]$ and by (5.24) the only contributions come from $\left(n_{1}=n_{3}\right.$ and $\left.n_{2}=n_{4}\right),\left(n_{1}=n_{2}\right.$ and $\left.n_{3}=n_{4}\right)$ and $\left(n_{2}=n_{3}\right.$ and $\left.n_{1}=n_{4}\right)$. Hence by symmetry and using that the fourth moments of the Gaussians $g_{n}(\omega)$ are bounded we have

$$
\left\|Y_{N, M}^{1}\right\|_{L^{2}}^{2}=\mathbb{E}\left[\left|Y_{N, M}^{1}\right|^{2}\right] \leq C \sum_{N \leq\left|n_{1}\right|,\left|n_{2}\right|<M} \frac{n_{1}^{2} n_{2}^{2}}{\left\langle n_{1}\right\rangle^{4}\left\langle n_{2}\right\rangle^{4}} \lesssim \frac{1}{N^{2}} .
$$

On the other hand, since

$$
\left|Y_{N, M}^{2}\right|^{2}=\sum_{N \leq\left|n_{1}\right|,\left|n_{2}\right|,\left|n_{3}\right|,\left|n_{4}\right|<M} n_{1} n_{2} n_{3} n_{4} \frac{\left(G_{n_{1}}+G_{n_{2}}\right)\left(G_{n_{3}}+G_{n_{4}}\right)}{\left\langle n_{1}\right\rangle^{2}\left\langle n_{2}\right\rangle^{2}\left\langle n_{3}\right\rangle^{2}\left\langle n_{4}\right\rangle^{2}},
$$

by symmetry it is enough to consider a single term of the form

$$
\sum_{N \leq\left|n_{1}\right|,\left|n_{2}\right|,\left|n_{3}\right|,\left|n_{4}\right|<M} n_{1} n_{2} n_{3} n_{4} \frac{G_{n_{j}} G_{n_{k}}}{\left\langle n_{1}\right\rangle^{2}\left\langle n_{2}\right\rangle^{2}\left\langle n_{3}\right\rangle^{2}\left\langle n_{4}\right\rangle^{2}},
$$

with $1 \leq j \neq k \leq 4$, which we set without any loss of generality to be $j=1, k=3$. We then have

$$
\left\|Y_{N, M}^{2}\right\|_{L^{2}}^{2}=\mathbb{E}\left[\left|Y_{N, M}^{2}\right|^{2}\right] \leq C \sum_{N \leq\left|n_{1}\right|,\left|n_{2}\right|,\left|n_{4}\right| \leq M} \frac{n_{1}^{2} n_{2} n_{4}}{\left\langle n_{1}\right\rangle^{4}\left\langle n_{2}\right\rangle^{2}\left\langle n_{4}\right\rangle^{2}}=0
$$

by symmetry. From (5.23) and (5.25) we obtain (5.20) as desired.

To prove (5.21) we use (5.22) to define

$$
S_{M, N}(v):=\left|X_{M}(v)-X_{N}(v)\right|^{2}=\sum_{N \leq\left|n_{1}\right|,\left|n_{2}\right|<M} n_{1} n_{2} \frac{\left|g_{n_{1}}(\omega)\right|^{2}\left|g_{n_{2}}(\omega)\right|^{2}}{\left\langle n_{1}\right\rangle^{2}\left\langle n_{2}\right\rangle^{2}}
$$

which fits the framework of (5.18) in Proposition 5.10 with $k=4$. Then it follows that for any $p \geq 2$,

$$
\left\|S_{M, N}(v)\right\|_{L^{p}} \lesssim(p-1)^{2}\left\|S_{M, N}(v)\right\|_{L^{2}}=(p-1)^{2} \| X_{M}(v)-X_{N}\left(v \|_{L^{4}}^{2} \lesssim(p-1)^{2} \frac{1}{N}\right.
$$

On the other hand if we set $q=2 p$, then by (5.27) we have

$$
\left\|X_{M}(v)-X_{N}(v)\right\|_{L^{q}}=\left\|S_{M, N}(v)\right\|_{L^{p}}^{1 / 2} \lesssim(q-1) \frac{1}{N^{1 / 2}},
$$

hence (5.21) for $q \geq 4$. Finally, Hölder's inequality gives (5.21) for $2 \leq q \leq 4$. 
Proof of Lemma 5.9. Let us denote $M_{N}(v):=\int_{\mathbb{T}}\left|v_{N}\right|^{2} d x$. Up to absolute constants we write

$$
\begin{aligned}
\rho\left(\left|K_{M}(v)-K_{N}(v)\right|>2 \gamma\right) \leq & \rho\left(\left|X_{M}(v) M_{M}(v)-X_{N}(v) M_{N}(v)\right|>\gamma\right) \\
& +\rho\left(\left|M_{M}(v)^{3}-M_{N}(v)^{3}\right|>\gamma\right) .
\end{aligned}
$$

Then

$\rho\left(\left|X_{M}(v) M_{M}(v)-X_{N}(v) M_{N}(v)\right|>\gamma\right)$

$\leq \rho\left(\left|X_{M}(v)-X_{N}(v)\right| M_{M}(v)>\gamma / 2\right)+\rho\left(\left|M_{M}(v)-M_{N}(v)\right|\left|X_{N}(v)\right|>\gamma / 2\right)=I_{1}+I_{2}$.

Let $\lambda>0$ to be determined. Then by (5.19), (5.6) and (5.17) with $p=2, \alpha=(1 / 2)-$, we have

$$
\begin{aligned}
I_{2} & \leq \rho\left(\left|X_{N}(v)\right|>\lambda\right)+\rho\left(\left|M_{M}(v)-M_{N}(v)\right|>\frac{\gamma}{2} \lambda^{-1}\right) \\
& \leq e^{-c \lambda N^{-2 \varepsilon}}+\rho\left(\left\|v^{N}-v^{M}\right\|_{L^{2}}>\frac{\gamma}{4 B} \lambda^{-1}\right) \leq e^{-c \lambda N^{-2 \varepsilon}}+e^{-c_{\gamma, B} N^{1-} \lambda^{-2}} .
\end{aligned}
$$

By setting $\lambda=N^{1 / 3+(2 \varepsilon / 3)-}$ we have $I_{2} \lesssim e^{-c_{\gamma, B} N^{1 / 3-(4 \varepsilon / 3)-}}$.

To estimate $I_{1}$ we first note that

$$
M_{M}(v) \leq\|v\|_{L^{2}}^{2} \leq B^{2} .
$$

Then by (5.21) and Chebyshev's inequality ${ }^{8}$ we have

$$
I_{1} \leq \rho\left(\left|X_{M}(v)-X_{N}(v)\right|>\frac{\gamma}{2 B^{2}}\right) \lesssim e^{-C_{B} N^{\frac{1}{2}} \gamma} .
$$

To estimate the second term of (5.28), we use (5.29) to obtain

$$
\rho\left(\left|M_{M}(v)^{3}-M_{N}(v)^{3}\right|>\gamma\right) \leq \rho\left(\left|M_{M}(v)-M_{N}(v)\right|>c_{B} \gamma\right) \leq e^{-C_{B} \gamma^{2} N^{1-}}
$$

by arguing as in the estimate for $I_{2}$ above.

Lemma 5.12. $R_{N}(v)$ converges in measure to $R(v)$.

Proof. If $\left\|P_{N} v\right\|_{L^{2}} \leq B$ for all $N \in \mathbb{N}$, then $\|v\|_{L^{2}} \leq B$. Hence, by continuity from above, we have, for $\delta \in(0,1)$,

$$
\begin{aligned}
\lim _{N \rightarrow \infty} \rho\left(\left\{v:\left|\chi_{\left\{\left\|v^{N}\right\|_{L^{2}} \leq B\right\}}-\chi_{\left\{\|v\|_{L^{2}} \leq B\right\}}\right|>\delta\right\}\right) & \\
= & \lim _{N \rightarrow \infty} \rho\left(\left\|v^{N}\right\|_{L^{2}} \leq B\right)-\rho\left(\|v\|_{L^{2}} \leq B\right) \\
& =\rho\left(\bigcap_{N=1}^{\infty}\left\{\left\|v^{N}\right\|_{L^{2}} \leq B\right\}\right)-\rho\left(\|v\|_{L^{2}} \leq B\right)=0 .
\end{aligned}
$$

Thus, $\chi_{\left\{\left\|v^{N}\right\|_{L^{2}} \leq B\right\}}$ converges to $\chi_{\left\{\|v\|_{L^{2}} \leq B\right\}}$ in measure. By Lemma 5.8(a), $F_{N}$ converges to $F$ in measure, and by Lemma 5.9, $K_{N}$ converges to $K$ in measure.

\footnotetext{
8 Cf. Lemma 4.5 in [46].
} 
Lastly, we consider $G_{N}(v)$ and show it is Cauchy in measure provided $\|v\|_{L^{2}} \leq B$. Assume $N \leq M$. Then

$$
\begin{aligned}
& 4 \pi G_{N}(v)-4 \pi G_{M}(v) \\
& =\left(\int_{\mathbb{T}}\left(\left|v^{M}\right|^{2}-\left|v^{N}\right|^{2}\right) d x\right)\left(\int_{\mathbb{T}}\left|v^{M}\right|^{4} d x\right)+\left(\int_{\mathbb{T}}\left|v^{N}\right|^{2} d x\right)\left(\int_{\mathbb{T}}\left(\left|v^{M}\right|^{4}-\left|v^{N}\right|^{4}\right) d x\right) \\
& \leq c_{B}\left\|v^{M}-v^{N}\right\|_{L^{2}}\left\|v^{M}\right\|_{L^{4}}^{4}+\left\|v^{N}\right\|_{L^{2}}^{2}\left|\left\|v^{M}\right\|_{L^{4}}^{4}-\left\|v^{N}\right\|_{L^{4}}^{4}\right| \\
& \leq C_{B}\left[\left\|v^{M}-v^{N}\right\|_{L^{2}}\left\|v^{M}\right\|_{L^{4}}^{4}+3\left(\left\|v^{M}\right\|_{L^{4}}^{3}+\left\|v^{N}\right\|_{L^{4}}^{3}\right)\left\|v^{M}-v^{N}\right\|_{L^{4}}\right] .
\end{aligned}
$$

Fix any $\gamma>0$; then

$$
\begin{aligned}
\rho\left(\left|4 \pi G_{M}(v)-4 \pi G_{N}(v)\right|>\gamma\right) \leq & \rho\left(\left\|v^{M}-v^{N}\right\|_{L^{2}}^{2}\left\|v^{M}\right\|_{L^{4}}^{4}>\frac{\gamma}{2 C_{B}}\right) \\
& +\rho\left(\left(\left\|v^{M}\right\|_{L^{4}}^{3}+\left\|v^{N}\right\|_{L^{4}}^{3}\right)\left\|v^{M}-v^{N}\right\|_{L^{4}}>\frac{\gamma}{6 C_{B}}\right) .
\end{aligned}
$$

To treat the first term we write

$$
\rho\left(\left\|v^{M}-v^{N}\right\|_{L^{2}}\left\|v^{M}\right\|_{L^{4}}^{4}>\frac{\gamma}{2 C_{B}}\right) \leq \rho\left(\left\|v^{M}-v^{N}\right\|_{L^{2}}>\lambda^{-1} \frac{\gamma}{2 C_{B}}\right)+\rho\left(\left\|v^{M}\right\|_{L^{4}}^{4}>\lambda\right)
$$

for some $\lambda>0$ to be determined. We use (5.15) with $\alpha=(1 / 2)-$ corresponding to $p=2$ and (5.14) to get

$$
\rho\left(\left\|v^{M}-v^{N}\right\|_{L^{2}}>c_{B} \gamma \lambda^{-1}\right) \leq e^{-c_{B}^{\prime} \gamma^{2} N^{1-} \lambda^{-2}}
$$

and

$$
\rho\left(\left\|v^{M}\right\|_{L^{4}}>\lambda^{1 / 4}\right) \leq e^{-c \lambda^{1 / 2}} .
$$

A decay of $e^{-C_{B} N^{(1 / 5)-} \gamma^{2 / 5}}$ follows by setting $\lambda=N^{(2 / 5)-} \gamma^{4 / 5}$.

For the second term write

$$
\begin{aligned}
& \rho\left(\left\|v^{M}-v^{N}\right\|_{L^{4}}\left(\left\|v^{M}\right\|_{L^{4}}^{3}+\left\|v^{N}\right\|_{L^{4}}^{3}\right)>\frac{\gamma}{6 C_{B}}\right) \\
& \quad \leq \rho\left(\left\|v^{M}-v^{N}\right\|_{L^{4}}>c_{B} \gamma \lambda^{-1}\right)+\rho\left(\left\|v^{M}\right\|_{L^{4}}>c_{1} \lambda^{1 / 3}\right)+\rho\left(\left\|v^{N}\right\|_{L^{4}}>c_{2} \lambda^{1 / 3}\right) \\
& \quad \leq e^{-c_{B}^{\prime} \gamma^{2} N^{\frac{1}{2}-} \lambda^{-2}}+2 e^{-c \lambda^{2 / 3}}
\end{aligned}
$$

since $\alpha=(1 / 4)-$ when $p=4$ in (5.15). A decay of $e^{-C_{B} N^{(1 / 8)-} \gamma^{1 / 2}}$ follows by setting $\lambda=N^{(3 / 16)-} \gamma^{3 / 4}$.

Thus, $G_{N}(v)$ converges to $G(v)$ in measure and hence, by composition and multiplication of continuous functions, $R_{N}(v)$ converges to $R(v)$ in measure.

The following proposition shows that the weight $R(v)$ is indeed integrable with respect to the Wiener measure $\rho$. 
Proposition 5.13. (a) For sufficiently small $B>0$, we have $R(v) \in L^{2}(d \rho)$. In particular, the weighted Wiener measure $\mu$ is a probability measure, absolutely continuous with respect to the Wiener measure $\rho$.

(b) We have the following tail estimate: Let $2 \leq r<\infty$ and $(s-1) r<-1$; then there exists a constant $c$ such that

$$
\mu\left(\|v\|_{\mathcal{F} L^{s, r}}>K\right) \leq e^{-c K^{2}}
$$

for sufficiently large $K>0$.

(c) The finite-dimensional weighted Wiener measure $\mu_{N}$ in (5.13) converges weakly to $\mu$.

Proof. (a) By Hölder's inequality, we have

$$
\begin{aligned}
& \int R_{N}^{2}(v) d \rho(v) \leq C_{B}\left(\int \chi_{\left\{\left\|v^{N}\right\|_{L^{2}} \leq B\right\}} e^{-3 \operatorname{Im} \int\left(v^{N}\right)^{2} \overline{v^{N}} \overline{v_{x}^{N}} d x} d \rho(v)\right)^{1 / 3} \\
& \times\left(\int \chi_{\left\{\left\|v^{N}\right\|_{\left.L^{2} \leq B\right\}}\right.} e^{\frac{3 B^{2}}{2 \pi}\left(\int\left|v^{N}\right|^{4} d x\right)} d \rho(v)\right)^{1 / 3} \\
& \times\left(\int \chi_{\left\{\left\|v^{N}\right\|_{L^{2}} \leq B\right\}} e^{-\frac{6}{\pi} M_{N}(v) \operatorname{Im} \int v^{N} \overline{v_{x}^{N}} d x} d \rho(v)\right)^{1 / 3} .
\end{aligned}
$$

It follows from Lemma 3.10 in [3] (see also [30]) that the second factor is finite for any $B>0$, whereas it was shown in [42, Proposition 4.2] that the first factor is finite for sufficiently small $B>0$. For the third factor we proceed as in the proof of [42, Proposition 4.2]. In what follows we always implicitly assume that $\left\|v_{N}\right\|_{L^{2}} \leq B$. If we define

$$
A_{\gamma, N}=\left\{\chi_{\left\{\left\|v^{N}\right\|_{L^{2}} \leq B\right\}} e^{-\frac{6}{\pi} M_{N}(v) \operatorname{Im} \int v^{N} \overline{v_{x}^{N}} d x}>\gamma\right\},
$$

then we need to show that

$$
\int_{0}^{\infty} \gamma^{2} \rho\left(A_{\gamma, N}\right) d \gamma
$$

is convergent uniformly with respect to $N$ for $B>0$ small enough. Let $N_{0}=\ln \gamma$ and assume first that $N \leq N_{0} \leq\left(C / B^{2}\right) \ln \gamma$ for $B$ small enough. We first observe that

$$
\left|M_{N}(v) \operatorname{Im} \int v^{N} \overline{v_{x}^{N}} d x\right| \leq C B^{2}\left\|\partial_{x}\left(v^{N}\right)^{2}\right\|_{L^{\infty}(\mathbb{T})} .
$$

We also note that

$$
\rho\left(A_{\gamma, N}\right) \leq \rho\left(\left|M_{N}(v) \operatorname{Im} \int v^{N} \overline{v_{x}^{N}} d x\right|>C \ln \gamma\right)
$$

combining (5.33) and (5.32) with Proposition 4.1 in [42], we can continue with

$$
\rho\left(A_{\gamma, N}\right) \leq \rho\left(\left\|\partial_{x}\left(v^{N}\right)^{2}\right\|_{L^{\infty}(\mathbb{T})}>C B^{-2} \ln \gamma\right) \lesssim e^{-\frac{C}{B^{2}} \ln \gamma}=\gamma^{-C / B^{2}},
$$

and the convergence of (5.32) follows by taking $B$ small enough. 
Assume now that $N>N_{0}=\ln \gamma$. Then we observe that $A_{\gamma, N} \subset B_{\gamma, N} \cup C_{\gamma, N}$ where

$$
B_{\gamma, N}:=\left\{\left|X_{N_{0}}(v)\right|>\frac{\pi}{12 B^{2}} \ln \gamma\right\}, \quad C_{\gamma, N}:=\left\{\left|X_{N}-X_{N_{0}}(v)\right|>\frac{\pi}{12 B^{2}} \ln \gamma\right\} .
$$

We first observe that from the argument above

$$
\rho\left(B_{\gamma, N}\right) \leq \rho\left(\left\|\partial_{x}\left(v^{N_{0}}\right)^{2}\right\|_{L^{\infty}(\mathbb{T})}>C B^{-2} \ln \gamma\right) \lesssim \gamma^{-C / B^{2}}
$$

On the other hand from (5.30) and the fact that $N>\ln \gamma$ we have

$$
\rho\left(C_{\gamma, N}\right) \lesssim e^{-C_{B} N^{1 / 2} \ln \gamma} \leq e^{-C_{B}(\ln \gamma)^{1+1 / 2}} \leq C_{B, L} \gamma^{L},
$$

for any $L \geq 1$ and an appropriate constant $C_{B, L}$ depending on $B$ and $L$. From this again the convergence of (5.32) follows.

Hence we see that $R_{N}(v) \in L^{2}(d \rho)$ for sufficiently small $B>0$, independent of $N$. Then, by Lemma 5.12 and Fatou's lemma, we obtain $R(v) \in L^{2}(d \rho)$.

(b) By the Cauchy-Schwarz inequality, we have

$$
\int \chi_{\left\{\|v\|_{\left.\mathcal{F} L^{s, r}>K\right\}}\right.} d \mu \leq\|R(v)\|_{L^{2}(d \rho)}\left\{\rho\left(\|v\|_{\mathcal{F} L^{s, r}}>K\right)\right\}^{1 / 2} .
$$

Then (5.31) follows from (5.6).

(c) Let us define

$$
\mathcal{H}:=\bigcup_{M}\left\{F: F=G\left(\widehat{v}_{-M}, \ldots, \widehat{v}_{M}\right), G \text { bounded and continuous }\right\}
$$

Note this is a dense set in $L^{1}\left(\mathcal{F} L^{s, r}, \mu\right)$ with $2 \leq r<\infty$ and $(s-1) r<-1$. Fix $F \in \mathcal{H}$; then $F$ depends on $M$, finitely many modes, for some $M$. Fix $\varepsilon>0$. Then, for $N>M$, we have

$$
\begin{aligned}
\mid \int F(v) d \mu_{N} & -\int F(v) d \mu|=| \int F(v)\left(R_{N}(v)-R(v)\right) d \rho \mid \\
\leq & \left|\int_{\left\{\left|R_{N}(v)-R(v)\right|<\varepsilon\right\}} F(v)\left(R_{N}(v)-R(v)\right) d \rho\right| \\
& +\left|\int_{\left\{\left|R_{N}(v)-R(v)\right| \geq \varepsilon\right\}} F(v)\left(R_{N}(v)-R(v)\right) d \rho\right| \\
\leq & \varepsilon \sup |F|+\sup |F|\left\|R_{N}(v)-R(v)\right\|_{L^{2}(d \rho)}\left\{\rho\left(\left|R_{N}(v)-R(v)\right| \geq \varepsilon\right)\right\}^{1 / 2} .
\end{aligned}
$$

From the proof of Proposition 5.13, we have $\left\|R_{N}(v)-R(v)\right\|_{L^{2}(d \rho)} \leq\left\|R_{N}(v)\right\|_{L^{2}(d \rho)}+$ $\|R(v)\|_{L^{2}(d \rho)}<C<\infty$ for all $N$. By Lemma 5.12, $\rho\left(\left|R_{N}(v)-R(v)\right| \geq \varepsilon\right) \rightarrow 0$ as $n \rightarrow \infty$. 
Now, let $F$ be a general bounded continuous function on $\mathcal{F} L^{s, r}$ with $2 \leq r<\infty$ and $(s-1) r<-1$. Let $F_{M}$ denote its restriction to $E_{M}$, i.e. $F_{M}(v)=F\left(v^{M}\right)$ where $v^{M}=P_{M} v$. By the Cauchy-Schwarz inequality, we have

$$
\begin{aligned}
\left|\int F(v) d \mu-\int F_{M}(v) d \mu\right| & =\left|\int\left(F(v)-F\left(v^{M}\right)\right) R(v) d \rho\right| \\
& \leq\|R(v)\|_{L^{2}(d \rho)}\left(\int\left|F(v)-F\left(v^{M}\right)\right|^{2} d \rho\right)^{1 / 2} .
\end{aligned}
$$

By continuity of $F$, given $\varepsilon>0$, there exists $\delta>0$ such that

$$
\left\|P_{M}^{\perp} v\right\|_{\mathcal{F} L^{s, r}}=\left\|v-v^{M}\right\|_{\mathcal{F} L^{s, r}}<\delta \Rightarrow\left|F(v)-F\left(v^{M}\right)\right|<\varepsilon .
$$

Then the contribution to (5.35) from $\left\{v:\left\|P_{M}^{\perp} v\right\|_{\mathcal{F} L^{s, r}}<\delta\right\}$ is at most $\varepsilon\|R(v)\|_{L^{2}(d \rho)}$. Without loss of generality, assume $\delta \leq \varepsilon^{2}$. By the measurability of the $\mathcal{F} L^{s, r}$-norm (see Definition 5.3), the contribution to (5.35) from $\left\{v:\left\|P_{M}^{\perp} v\right\|_{\mathcal{F} L^{s, r}} \geq \delta\right\}$ is at most

$$
\begin{aligned}
2 \sup |F| \cdot\|R(v)\|_{L^{2}(d \rho)} & \left\{\rho\left(\left\|P_{M}^{\perp} v\right\|_{\mathcal{F} L^{s, r}} \geq \delta\right)\right\}^{1 / 2} \\
\leq & 2 \sup |F| \cdot\|R(v)\|_{L^{2}(d \rho)} \delta^{1 / 2} \leq 2 \sup |F| \cdot\|R(v)\|_{L^{2}(d \rho)} \varepsilon
\end{aligned}
$$

for sufficiently large $M$. A similar argument can be used to show $\mid \int F(v) d \mu_{N}-$ $\int F_{M}(v) d \mu_{N} \mid \leq C(f, R) \varepsilon$, independent of $N$. Hence, $\mu_{N}$ converges weakly to $\mu$.

Remark 5.14. A tail estimate similar to (5.31) holds for the finite-dimensional weighted Wiener measure $\mu_{N}$, i.e. we have

$$
\mu_{N}\left(\left\|v^{N}\right\|_{\mathcal{F} L^{s, r}}>K\right) \leq e^{-c K^{2}},
$$

where the constant is independent of $N$.

Remark 5.15. The measure $\rho_{N}$ is not absolutely continuous with respect to $\mu_{N}$ but its restriction to $\left\{\left\|v^{N}\right\|_{L^{2}} \leq B\right\}$, i.e., $\widetilde{\rho}_{N}=\widehat{Z}_{N}^{-1} \chi_{\left\{\left\|v^{N}\right\|_{L^{2}} \leq B\right\}} \rho_{N}$ is absolutely continuous with respect to $\mu_{N}$, and from (5.13) we have

$$
\frac{d \widetilde{\rho}_{N}}{d \mu_{N}}:=\tilde{R}_{N}=\tilde{Z}_{N}^{-1} \chi_{\left\{\left\|v^{N}\right\|_{L^{2}} \leq B\right\}} e^{\frac{1}{2} \mathcal{N}\left(v^{N}\right)}
$$

for a suitable renormalization $\tilde{Z}_{N}$. Since $\mathcal{N}\left(v^{N}\right)$ does not have a definite sign, Lemma 5.8, Lemma 5.12 and Proposition 5.13(a) hold for $\tilde{R}_{N}$ and its corresponding limit $\tilde{R}$. In particular, for sufficiently small $B, \tilde{R}_{N} \in L^{2}(d \rho)$ for all $N$ with bound independent of $N$. The latter fact will be used in the proof of Proposition 6.2 below.

Remark 5.16. Given any $p<\infty$, one can prove $R(v) \in L^{p}(d \rho)$ for sufficiently small $B \leq B(p)$. However, $B(p) \rightarrow 0$ as $p \rightarrow \infty$, i.e. there is no uniform lower bound on the size of the $L^{2}$-cutoff. For our purpose, the integrability with $p=2$ suffices. 


\section{Almost sure well-posedness of FGDNLS and invariance of the measure}

In order to prove the global well-posedness of $\mu$-almost all solutions of FGDNLS (3.1) we fix once again $s=(2 / 3)-$ and $r=3$ so that we have at our disposal the local wellposedness result in $\mathcal{F} L^{s, r}$, that the measure is supported on $\mathcal{F} L^{s, r}$, and also the energy growth estimates in Theorem 4.2 as explained in Remark 4.3.

We first use the almost invariance of the finite-dimensional measure $\mu_{N}$ under the flow of the truncated equation (3.1) to control the growth of solutions.

Lemma 6.1. For any given $T>0$ and $\varepsilon>0$ there exists an integer $N_{0}=N_{0}(T, \varepsilon)$ and sets $\widetilde{\Omega}_{N}=\widetilde{\Omega}_{N}(\varepsilon, T) \subset \mathbb{R}^{4 N+2}$ such that for $N>N_{0}$ :

(a) $\mu_{N}\left(\widetilde{\Omega}_{N}\right) \geq 1-\varepsilon$.

(b) For any initial condition $v_{0}^{N} \in \widetilde{\Omega}_{N}, F G D N L S$ (3.1) is well-posed on $[-T, T]$ and its solution $v^{N}(t)$ satisfies the bound

$$
\sup _{|t| \leq T}\left\|v^{N}(t)\right\|_{\mathcal{F} L^{\frac{2}{3}-, 3}} \lesssim\left(\log \frac{T}{\varepsilon}\right)^{1 / 2} .
$$

Proof. It is enough to consider $t \in[0, T]$; the argument for $t \in[-T, 0]$ is similar. We set

$$
C_{N}(K, B):=\left\{w^{N} \in \mathbb{R}^{4 N+2}:\left\|w^{N}\right\|_{\mathcal{F}^{L^{3}-, 3}} \leq K,\left\|w^{N}\right\|_{L^{2}} \leq B\right\} .
$$

If the initial condition $v_{0}^{N}$ is in $C_{N}(K, B)$ then FGDNLS (3.1) is locally well-posed on the time interval of length $\delta \sim K^{-\gamma}$ by Theorem 3.2, where $\gamma>0$ is independent of $N$. Furthermore, if $\mu_{N}$ is given by (5.13), then for sufficiently large $K$ we have $\mu_{N}\left(C_{N}(K, B)^{c}\right) \leq e^{-c K^{2}}$ for some constant $c$ which is independent of $N$ by (5.36).

Let $\Phi_{N}(t)$ be the flow map of (3.1). We define

$$
\widetilde{\Omega}_{N}:=\left\{v_{0}^{N}: \Phi_{N}(j \delta)\left(v_{0}^{N}\right) \in C_{N}(K, B), j=0,1, \ldots,[T / \delta]\right\} .
$$

Note that $\widetilde{\Omega}_{N}^{c}=\bigcup_{k=0}^{[T / \delta]} D_{k}$, where

$$
\begin{aligned}
D_{k} & =\left\{v_{0}^{N} ; k=\min \left\{j: \Phi_{N}(j \delta)\left(v_{0}^{N}\right) \in C_{N}(K, B)^{c}\right\}\right\}, \\
& =\left[\bigcap_{j=0}^{k-1} \Phi_{N}(-j \delta)\left(C_{N}(K, B)\right)\right] \cap \Phi_{N}(-k \delta)\left(C_{N}(B, K)^{c}\right) .
\end{aligned}
$$

One verifies easily that the sets $D_{k}$ satisfy

$$
D_{0}=C_{N}(K, B)^{c}, \quad D_{k}=C_{N}(K, B) \cap \Phi_{N}(-\delta)\left(D_{k-1}\right) .
$$

By Lemma 4.1, the Lebesgue measure $d \mu_{N}^{0} \equiv \prod_{|n| \leq N} d a_{n} d b_{n}$ is invariant under the flow $\Phi_{N}(t)$ (i.e. for any $f \in L^{1}\left(d \mu_{N}^{0}\right)$ we have $\left.\int f \circ \Phi_{N}(t) d \mu_{N}^{0}=\int f d \mu_{N}^{0}\right)$. 
Using the energy growth estimate ${ }^{9}$ in Theorem 4.2 and the invariance of the $L^{2}$ norm $m(v)=\frac{1}{2 \pi}\|v\|_{L^{2}}$ under $\Phi_{N}(t)$ (i.e. $m \circ \Phi_{N}(t)=m$ for all $t$; see Remark 3.1) we have, for any set $A \subset \mathbb{R}^{4 N+2}$,

$$
\begin{aligned}
\mu_{N}\left(C_{N}(K, B) \cap A\right) & =Z_{N}^{-1} \int \chi_{\left\{C_{N}(K, B) \cap A\right\}} \chi_{\left\{m \leq 2 \pi B^{2}\right\}} e^{-\frac{1}{2} \mathcal{E}-\pi m} d \mu_{N}^{0} \\
& =Z_{N}^{-1} \int \chi_{\left\{C_{N}(K, B) \cap A\right\}} \circ \Phi_{N}(-\delta) \chi_{\left\{m \leq 2 \pi B^{2}\right\}} e^{-\mathcal{E}_{\circ} \Phi_{N}(-\delta)-\pi m} d \mu_{N}^{0} \\
& =\int \chi_{\left\{\Phi_{N}(\delta)\left(C_{N}(K, B) \cap A\right)\right\}} e^{-\frac{1}{2}\left(\mathcal{E} \circ \Phi_{N}(-\delta)-\mathcal{E}\right)} d \mu_{N} \\
& \leq e^{c(\delta) N^{-\beta} K^{8}} \mu_{N}\left(\Phi_{N}(\delta)\left(C_{N}(K, B) \cap A\right)\right) \\
& \leq e^{c(\delta) N^{-\beta} K^{8}} \mu_{N}\left(\Phi_{N}(\delta)(A)\right)
\end{aligned}
$$

Applying (6.3) to (6.2) with $A=\Phi_{N}(-\delta)\left(D_{k-1}\right)$ and iterating in $k \in\{0, \ldots,[T / \delta]\}$, we obtain

$$
\mu_{N}\left(D_{K}\right) \leq e^{c(\delta) N^{-\beta} K^{8}} \mu_{N}\left(D_{K-1}\right) \leq e^{k c(\delta) N^{-\beta} K^{8}} e^{-c K^{2}}
$$

and thus

$$
\mu_{N}\left(\widetilde{\Omega}_{N}^{c}\right) \leq \sum_{k=0}^{[T / \delta]} e^{k c(\delta) N^{-\beta} K^{8}} e^{-c K^{2}} \lesssim\left[\frac{T}{\delta}\right] e^{-c K^{2}} \sim T K^{\gamma} e^{-c K^{2}}
$$

for $N \geq N_{0}(T, K)$. By choosing $K \sim(\log (T / \varepsilon))^{1 / 2}$, we have $\mu_{N}\left(\widetilde{\Omega}_{N}^{c}\right)<\varepsilon$ as desired.

Finally, by construction, we have $\left\|v^{N}(j \delta)\right\|_{\mathcal{F} L^{(2 / 3)-, 3}} \leq K$ for $j=0, \ldots,[T / \delta]$ and by the local theory, we have

$$
\sup _{0 \leq t \leq T}\left\|v^{N}(t)\right\|_{\mathcal{F} L^{\frac{2}{3}-, 3}} \leq 2 K \sim\left(\log \frac{T}{\varepsilon}\right)^{1 / 2} .
$$

Combining Lemma 6.1 with the approximation Lemma 3.3 we can now prove a similar result for the solution of the initial value problem GDNLS (2.6).

Proposition 6.2. For any given $T>0$ and $\varepsilon>0$ there exists a set $\Omega(\varepsilon, T)$ such that:

(a) $\mu(\Omega(\varepsilon, T)) \geq 1-\varepsilon$.

(b) For any initial condition $v_{0} \in \Omega(\varepsilon, T)$ the initial value problem GDNLS (2.6) is well-posed on $[-T, T]$ with the bound

$$
\sup _{|t| \leq T}\|v(t)\|_{\mathcal{F}^{2} \frac{2}{3}-3} \lesssim\left(\log \frac{T}{\varepsilon}\right)^{1 / 2} .
$$

\footnotetext{
9 Without loss of generality we assume $\max \left(K^{6}, K^{8}\right)=K^{8}$ in Theorem 4.2.
} 
Proof. Let $\widetilde{\Omega}_{N}=\widetilde{\Omega}_{N}(\varepsilon, T)$ be the set given in Lemma 6.1 for $N \geq N_{0}(\varepsilon, T)$. This set is defined in terms of $K \sim(\log (T / \varepsilon))^{1 / 2}$ and for that same $K$ we define

$$
\Omega_{N}:=\Omega_{N}(\varepsilon, T):=\left\{v_{0} \in \mathcal{F} L^{\frac{2}{3}-, 3}:\left\|v_{0}\right\|_{\mathcal{F} L^{\frac{2}{3}-, 3}} \leq K, P_{N} v_{0} \in \widetilde{\Omega}_{N}\right\}
$$

If $v_{0} \in \Omega_{N}$ then by Lemma 6.1 we have

$$
\sup _{t \leq T}\left\|\Phi_{N}(t)\left(P_{N} v_{0}\right)\right\|_{\mathcal{F} L^{\frac{2}{3}-, 3}} \leq 2 K .
$$

On the other hand for $v_{0} \in \Omega_{N}$ the local well-posedness theorem in [22] gives a $\delta>0$ and a solution $v(t)$ of GDNLS (2.6) for $|t| \leq \delta$.

By (3.5) in the proof of Lemma 3.3, with $K$ in place of $A$, we find that for every $s_{1}<(2 / 3)-$,

$$
\left\|v(\delta)-v^{N}(\delta)\right\|_{\mathcal{F} L^{s_{1}, 3}} \lesssim K N^{s_{1}-\frac{2}{3}+} .
$$

By choosing a larger $N_{0}$ if necessary, so that $[T / \delta] K N^{s_{1}-(2 / 3)+} \ll 1$ for $N>N_{0}$ we can repeat this argument $[T / \delta]$ times over the intervals $[j \delta,(j+1) \delta], j=0,1, \ldots,[T / \delta]-1$, to obtain

$$
\left\|v(j \delta)-v^{N}(j \delta)\right\|_{\mathcal{F} L^{s_{1}, 3}}<1 .
$$

Then from (6.4) and (6.5) we conclude

$$
\|v(t)\|_{\mathcal{F} L^{s_{1}, 3}} \lesssim 2 K+1 \sim\left(\log \frac{T}{\varepsilon}\right)^{1 / 2},
$$

and since the right hand side is independent of $s_{1}<(2 / 3)-$, we obtained the desired estimate.

To estimate $\mu\left(\Omega_{N}\right)$ note first that

$$
\Omega_{N}^{c} \subset\left\{v_{0} \in \mathcal{F} L^{\frac{2}{3}-, 3}:\left\|v_{0}\right\|_{\mathcal{F} L^{\frac{2}{3}-, 3}} \geq K\right\} \cup\left\{v_{0} \in \mathcal{F} L^{\frac{2}{3}-, 3}: P_{N} v_{0} \in \widetilde{\Omega}_{N}^{c}\right\}
$$

The first set on the right hand side of (6.6) has $\mu$ measure less than $\varepsilon$ by the tail bound in Proposition 5.13. The set $F_{N} \equiv\left\{v_{0} \in \mathcal{F} L^{(2 / 3)-, 3}: P_{N} v_{0} \in \widetilde{\Omega}_{N}^{c}\right\}$ is a cylinder set and we have $F_{N} \cap E_{N}=\widetilde{\Omega}_{N}^{c}$ (recall $\left.E_{N}=\operatorname{span}\left\{e^{i n x}\right\}_{|n| \leq N}\right)$. Thus $\rho\left(F_{N}\right)=\rho_{N}\left(F_{N}\right)=$ $\rho_{N}\left(\widetilde{\Omega}_{N}^{c}\right)$. On the other hand, recall that $\mu \ll \rho$ and that $\widetilde{\rho}_{N}$, the restriction of $\rho_{N}$ to the ball $\left\{\left\|v^{N}\right\|_{L^{2}} \leq B\right\}$, is absolutely continuous with respect to $\mu_{N}$ (see Remark 5.15). Then using Cauchy-Schwarz repeatedly we obtain

$$
\begin{aligned}
\mu\left(F_{N}\right) & \leq\left(\int R^{2} d \rho\right)^{1 / 2}\left(\int_{\tilde{\Omega}_{N}^{c}} \chi_{\left\{\left\|v^{N}\right\|_{L^{2}} \leq B\right\}} d \rho_{N}\right)^{1 / 2} \\
& \leq\left(\int R^{2} d \rho\right)^{1 / 2}\left(\int \tilde{R}_{N}^{2} d \mu_{N}\right)^{1 / 4} \mu_{N}\left(\tilde{\Omega}_{N}^{c}\right)^{1 / 4} \\
& \leq\left(\int R^{2} d \rho\right)^{1 / 2}\left(\int \tilde{R}_{N} d \rho_{N}\right)^{1 / 4} \mu_{N}\left(\tilde{\Omega}_{N}^{c}\right)^{1 / 4}
\end{aligned}
$$


where $\tilde{R}_{N}$ is as defined in Remark 5.15 and where in the last inequality we have used that by definition $\tilde{R}_{N}^{2} R_{N}=\tilde{R}_{N}$.

By relying on Lemma 5.12, Proposition 5.13 and Remark 5.15 we can bound the first two terms in (6.7) by a constant independent of $N$. This combined with Lemma 6.1 allows us to conclude that there exist a constant $d>0$ and $N_{1}(\varepsilon, T)$ such that $\mu\left(F_{N}\right) \leq d \varepsilon$ for $N \geq N_{1}$. So for $N \geq \max \left(N_{0}, N_{1}\right)$, any set $\Omega(\varepsilon, T):=\Omega_{N}(\varepsilon, T)$ satisfies the desired hypothesis.

Theorem 6.3 (Almost sure global well-posedness). There exists a subset $\Omega$ of the space $\mathcal{F} L^{(2 / 3)-, 3}$ with $\mu\left(\Omega^{c}\right)=0$ such that for every $v_{0} \in \Omega$ the initial value problem $G D N L S$ (2.6) with initial data $v_{0}$ is globally well-posed.

Proof. Fix an arbitrary $T$ and let $\varepsilon=2^{-i}$. Using the sets given in Proposition 6.2 we set

$$
\Omega(T):=\bigcup_{i} \Omega\left(2^{-i}, T\right) .
$$

If $v_{0} \in \Omega(T)$ then the initial value problem GDNLS (2.6) is well-posed up to time $T$. Since $\mu(\Omega(T)) \geq 1-2^{-i}$ for any $i \in \mathbb{N}$, the set $\Omega(T)$ has full measure.

Finally by taking $T:=2^{j}$ the set

$$
\Omega=\bigcap_{j} \Omega\left(2^{j}\right)
$$

also has full measure and if $v_{0} \in \Omega$ then the initial value problem GDNLS (2.6) is globally well-posed.

Remark 6.4. We note that by slightly modifying the proof of Theorem 6.3 above we could also derive a logarithmic bound in time on solutions similar to the one in [3] and [12].

Now that we have a well-defined flow on the measure space $\left(\mathcal{F} L^{(2 / 3)-, 3}, \mu\right)$, we show that $\mu$ is invariant under the flow $\Phi(t)$, following the argument in [38].

Theorem 6.5. The measure $\mu$ is invariant under the flow $\Phi(t)$.

Proof. Let us consider the measure space $\left(\mathcal{F} L^{(2 / 3)-, 3}, \mu\right)$. We need to show that for any measurable $A$ we have $\mu(A)=\mu(\Phi(-t)(A))$ for all $t \in \mathbb{R}$. Note that by the group property of the flow without loss of generality we can assume that $|t| \leq \delta$. An equivalent characterization of invariance is that for all $F \in L^{1}\left(\mathcal{F} L^{(2 / 3)-, 3}, \mu\right)$ we have

$$
\int F(\Phi(t)(v)) d \mu=\int F(v) d \mu .
$$

By an elementary approximation argument it is enough to show (6.9) for $F$ in a dense set in $L^{1}\left(\mathcal{F} L^{(2 / 3)-, 3}, \mu\right)$ which we choose as in (5.34) to be

$$
\mathcal{H}:=\bigcup_{M}\left\{F: F=G\left(\widehat{v}_{-M}, \ldots, \widehat{v}_{M}\right), G \text { bounded and continuous }\right\} .
$$


For $F \in \mathcal{H}$ choose an arbitrary $\epsilon>0$ and assume $N \geq M$. By Proposition 5.13, $\mu_{N}$ converges weakly to $\mu$ and thus

$$
\left|\int F d \mu-\int F d \mu_{N}\right|+\left|\int F \circ \Phi(t) d \mu-\int F \circ \Phi(t) d \mu_{N}\right| \leq \epsilon .
$$

Let $\Phi_{N}(t)$ be the flow map for FGDNLS (3.1). For $s_{1}<(2 / 3)-$, by Lemma 3.3, we deduce that $\left\|\Phi(t)(v)-\Phi_{N}(t)(v)\right\|_{\mathcal{F} L^{s_{1}, 3}}$ converges to 0 uniformly on $\{v$ : $\left.\|v\|_{\mathcal{F}_{L^{(2 / 3)-, 3}}} \leq K\right\}$. Using the tail estimate $\mu_{N}\left(\left\|v^{N}\right\|_{\mathcal{F} L^{(2 / 3)-, 3}}>K\right) \leq e^{-c K^{2}}$ (uniformly in $N$ ) and the continuity of $F$ in $\mathcal{F} L^{s_{1}, 3}$ we obtain

$$
\left|\int F \circ \Phi(t) d \mu_{N}-\int F \circ \Phi_{N}(t) d \mu_{N}\right| \leq 2\|F\|_{\infty} e^{-c K^{2}}+\epsilon \leq 3 \epsilon
$$

for large enough $K$ and $N$.

Finally using again the tail estimate for $\mu_{N}$, the invariance of Lebesgue measure under $\Phi_{N}(t)$ and the energy estimate given in Theorem 4.2 we obtain

$$
\begin{aligned}
& \left|\int F \circ \Phi_{N}(t) d \mu_{N}-\int F d \mu_{N}\right| \\
& \quad \leq 2\|F\|_{L^{\infty}} e^{-c K^{2}}+\left|\int_{\left\{\|v\|_{\mathcal{F} L^{\frac{2}{3}-, 3}} \leq K\right\}} F\left[e^{-\frac{1}{2}\left(\mathcal{E}_{\circ} \Phi_{N}(-t)-\mathcal{E}\right)}-1\right] d \mu_{N}\right| \\
& \quad \leq 2 \epsilon+\|F\|_{L^{\infty}\left(e^{c(\delta) N^{-\beta} K^{8}}-1\right) \leq 3 \epsilon}
\end{aligned}
$$

for sufficiently large $N$. By combining (6.10)-(6.12) we obtain invariance.

\section{The ungauged DNLS equation}

Recall that if $u(t, x)$ is a solution of DNLS (2.1) then $w(t, x)=G(u(t, x))$ where $G(f)(x)=\exp (-i J(f)) f(x)($ see $(2.5))$ is a solution of

$$
w_{t}-i w_{x x}-2 m(w) w_{x}=-w^{2} \overline{w_{x}}+\frac{i}{2}|w|^{4} w-i \psi(w) w-i m(w)|w|^{2} w
$$

with initial data $w(0)=G(u(0))$. Furthermore $v(t, x)=w(t, x-2 t m(w))$ is a solution of (2.6) with initial condition $v(0)=w(0)$. If $\Phi(t)$ denotes the flow map for GDNLS (2.6), let $\widetilde{\Phi}(t)$ denote the flow map of (7.1) and let $\Psi(t)$ denote the flow map of (2.1).

Clearly we have the relation

$$
\Psi(t)=G^{-1} \circ \widetilde{\Phi}(t) \circ G .
$$

To elucidate the relation between $\Phi(t)$ and $\widetilde{\Phi}(t)$ let $\tau_{\alpha}(s)$ denote the action of the group of spatial translations on functions, i.e., $\left(\tau_{\alpha}(s) w\right)(x):=w(x-\alpha s)$. We define a state dependent translation

$$
(\Gamma(s) w)(x):=\left(\tau_{2 m(w)}(s) w\right)(x)=w(x-2 s m(w)) .
$$


Note that the $H^{s}, L^{p}$ and $\mathcal{F} L^{s, r}$ norms are all invariant under this transformation. Furthermore we have

$$
v(t, x):=(\Gamma(t) w)(t, x)
$$

Since $m$ is preserved under $G, \Gamma(s)$ and both flows $\Psi(t)$ and $\widetilde{\Phi}(t)$, we have the relation

$$
\Phi(t)=\Gamma(t) \widetilde{\Phi}(t)=\widetilde{\Phi}(t) \Gamma(t),
$$

in particular $\widetilde{\Phi}(t)$ and $\Gamma(t)$ commute.

Finally if $\mu$ is a measure on $\Omega$ as in Theorem 6.3 and $\varphi: \Omega \rightarrow \Omega$ is a measurable map then we define the measure $v=\mu \circ \varphi^{-1}$ by

$$
v(A):=\mu\left(\varphi^{-1}(A)\right)=\mu(\{x: \varphi(x) \in A\}) .
$$

for all measurable sets $A$ or equivalently by

$$
\int F d \nu=\int F \circ \varphi d \mu
$$

for integrable $F$.

Consider the measure defined by

$$
v:=\mu \circ G
$$

Since the measure $\mu$ constructed in Proposition 5.13 is invariant under the flow $\Phi(t)$ we show that the flow $\Psi(t)$ for DNLS is well defined $v$ almost surely and that $v$ is invariant under the flow $\Psi(t)$.

Theorem 7.1 (Almost sure global well-posedness for DNLS). There exists a subset $\Sigma$ of the space $\mathcal{F} L^{(2 / 3)-, 3}$ with $v\left(\Sigma^{c}\right)=0$ such that for every $u_{0} \in \Sigma$ the IVP DNLS (2.1) with initial data $u_{0}$ is globally well-posed.

Proof. Let $\Omega$ be the set of full $\mu$ measure given in Theorem 6.3 and let $\Sigma=G^{-1}(\Omega)$. Note that $\Sigma$ is a set of full $v$ measure by (7.4). For $v_{0} \in \Omega$ the IVP GDNLS (2.6) with initial data $v_{0}$ is globally well-posed. Hence since the map $\mathcal{G}: C\left([-T, T] ; \mathcal{F} L^{s, r}\right) \rightarrow$ $C\left([-T, T] ; \mathcal{F} L^{s, r}\right)$ is a homeomorphism if $s>1 / 2-1 / r$ when $2<r<\infty$, the IVP (DNLS) (2.1) with initial data $u_{0}=G^{-1}\left(v_{0}\right)$ is also globally well-posed.

Finally we show that the measure $v$ is invariant under the flow map of DNLS (2.1).

Theorem 7.2. The measure $v=\mu \circ G$ is invariant under the flow $\Psi(t)$.

Proof. First we note that the measure $\mu$ is invariant under $\Gamma(t)$. The density of $\mu$ with respect to $\rho$ is $R(v)$ (see (5.12)), and it is verified easily that $R \circ \Gamma(t)=R$. Furthermore one also verifies easily that the finite-dimensional measures $\rho_{N}$ are also invariant under $\Gamma(t)$. As a consequence, since $\mu$ is invariant under $\Phi(t)$ by Theorem $6.5, \mu$ is also invariant under $\widetilde{\Phi}(t)$ because of (7.3). Finally $v$ is invariant under $\Psi(t)$ since by (7.2),

$$
\begin{aligned}
\int F \circ \Psi(t) d v & =\int F \circ G^{-1} \circ \widetilde{\Phi}(t) \circ G d \mu \circ G=\int F \circ G^{-1} \circ \widetilde{\Phi}(t) d \mu \\
& =\int F \circ G^{-1} d \mu=\int F d \nu .
\end{aligned}
$$


Acknowledgments. Andrea R. Nahmod and Gigliola Staffilani would like to warmly thank the Radcliffe Institute for Advanced Study at Harvard University for its wonderful hospitality while part of this work was being carried out. They also thank their fellow Fellows for the stimulating environment they created.

The first author is funded in part by NSF DMS 0803160 and a 2009-2010 Radcliffe Institute for Advanced Study Fellowship.

The third author is funded in part by NSF DMS 0605058.

The fourth author is funded in part by NSF DMS 0602678 and a 2009-2010 Radcliffe Institute for Advance Study Fellowship.

\section{References}

[1] Bényi, Á., Oh, T.: Modulation spaces, Wiener amalgam spaces, and Brownian motions. Adv. Math. 228, 2943-2981 (2011) Zbl 1229.42021 MR 2838066

[2] Biagioni, H. A., Linares, F.: Ill-posedness for the derivative Schrödinger and generalized Benjamin-Ono equations. Trans. Amer. Math. Soc. 353, 3649-3659 (2001) Zbl 0970.35154 MR 1837253

[3] Bourgain, J.: Periodic nonlinear Schrödinger equation and invariant measures. Comm. Math. Phys. 166, 1-26 (1994) Zbl 0822.35126 MR 1309539

[4] Bourgain, J.: Nonlinear Schrödinger equations. In: Hyperbolic Equations and Frequency Interactions (Park City, UT, 1995), IAS/ Park City Math. Ser. 5, Amer. Math. Soc., 3-157 (1999) Zbl 0952.35127 MR 1662829

[5] Bourgain, J.: Global Solutions of Nonlinear Schrödinger Equations. Amer. Math. Soc. Colloq. Publ. 46, Amer. Math. Soc. (1999) Zbl 0933.35178 MR 1691575

[6] Bourgain, J.: Refinements of Strichartz inequality and applications to $2 d$ NLS with critical nonlinearity. Int. Math. Res. Notices 1998, 253-284 Zbl 0917.35126 MR 1616917

[7] Bourgain, J.: Invariant measures for the 2D defocusing nonlinear Schrödinger equation. Comm. Math. Phys. 176, 421-445 (1996) Zbl 0852.35131 MR 1374420

[8] Bourgain, J.: Invariant measures for the Gross-Pitaevskii equation. J. Math. Pures Appl. 76, 649-702 (1997) Zbl 0906.35095 MR 1470880

[9] Bourgain, J.: On the Cauchy problem and invariant measure problem for the periodic Zakharov system. Duke Math. J. 76, 175-202 (1994) Zbl 0821.35120 MR 1301190

[10] Bourgain, J.: Fourier transform restriction phenomena for certain lattice subsets and applications to nonlinear evolution equations. I. Schrödinger equations. Geom. Funct. Anal. 3, 107-156 (1993) Zbl 0787.35097 MR 1209299

[11] Burq, N., Thomann, L., Tzvetkov, N.: Long time dynamics for the one dimensional nonlinear Schrödinger equation. arXiv:1002.4054v1 [math.AP]

[12] Burq, N., Tzvetkov, N.: Invariant measure for a three dimensional nonlinear wave equation. Int. Math. Res. Notices 2007, art. ID rnm108, 26 pp. Zbl 1134.35076 MR 2376217

[13] Burq, N., Tzvetkov, N.: Random data Cauchy theory for supercritical wave equations. II. A global theory. Invent. Math. 173, 477-496 (2008) Zbl 1187.35233 MR 2425134

[14] Christ, M: Power series solution of a nonlinear Schrödinger equation. In: Mathematical Aspects of Nonlinear Dispersive Equations, Ann. of Math. Stud. 163, Princeton Univ. Press, Princeton, NJ, 131-155 (2007) Zbl 1142.35084 MR 2333210

[15] Colliander, J., Keel, M., Staffilani, G., Takaoka, H., Tao, T.: Global well-posedness for Schrödinger equations with derivative. SIAM J. Math. Anal. 33, 649-669 (2001) Zbl 1002.35113 MR 1871414 
[16] Colliander, J., Keel, M., Staffilani, G., Takaoka, H., Tao, T.: A refined global wellposedness for Schrödinger equations with derivative. SIAM J. Math. Anal. 34, 64-86 (2002) Zbl 1034.35120 MR 1950826

[17] Colliander, J., Keel, M., Staffilani, G., Takaoka, H., Tao, T.: Sharp global well-posedness for $\mathrm{KdV}$ and modified $\mathrm{KdV}$ on $\mathbb{R}$ and $\mathbb{T}$. J. Amer. Math. Soc. 16, 705-749 (2003) Zbl 1025.35025 MR 1969209

[18] De Silva, D., Pavlovic, N., Staffilani, G., Tzirakis, N.: Global well-posedness for a periodic nonlinear Schrödinger equation in 1D and 2D. Discrete Contin. Dynam. Systems 19, 37-65 (2007) Zbl pre05236234 MR 2318273

[19] Fernique, M. X.: Intégrabilité des vecteurs gaussiens. C. R. Acad. Sci. Paris 270, 1698-1699 (1970) Zbl 0206.19002 MR 0266263

[20] Gross, L.: Abstract Wiener spaces. In: Proc. 5th Berkeley Sympos. Math. Statist. Probab. 2, Univ. California Press, Berkeley, CA, 31-42 (1965) Zbl 0187.40903 MR 0212152

[21] Grünrock, A.: Bi- and trilinear Schrödinger estimates in one space dimension with applications to cubic NLS and DNLS. Int. Math. Res. Notices 2005, 2525-2558 Zbl 1088.35063 MR 2181058

[22] Grünrock, A., Herr, S.: Low regularity local well-posedness of the derivative nonlinear Schrödinger equation with periodic initial data. SIAM J. Math. Anal. 39, 1890-1920 (2008) Zbl 1156.35471 MR 2390318

[23] Hayashi, N.: The initial value problem for the derivative nonlinear Schrödinger equation in the energy space. Nonlinear Anal. 20, 823-833 (1993) Zbl 0787.35099 MR 1214746

[24] Hayashi, N., Ozawa, T.: On the derivative nonlinear Schrödinger equation. Phys. D 55, 14-36 (1992) Zbl 0741.35081 MR 1152001

[25] Hayashi, N., Ozawa, T.: Finite energy solutions of nonlinear Schrödinger equation of derivative type. SIAM J. Math. Anal. 25, 1488-1503 (1994) Zbl 0809.35124 MR 1302158

[26] Herr, S.: On the Cauchy problem for the derivative nonlinear Schrödinger equation with periodic boundary condition. Int. Math. Res. Notices 2006, art. ID 96763, 33 pp. Zbl 1149.35074 MR 2219223

[27] Hörmander, L.: The Analysis of Linear Partial Differential Operators. II. Grundlehren Math. Wiss. 257, Springer, Berlin (1983) Zbl 0521.35002 MR 0705278

[28] Kaup, D. J., Newell, A. C.: An exact solution for the derivative nonlinear Schrödinger equation. J. Math. Phys. 19, 798-801 (1978) Zbl 0383.35015 MR 0464963

[29] Kuo, H.: Gaussian Measures in Banach Spaces. Lecture Notes in Math. 463, Springer, New York (1975) Zbl 0306.28010 MR 0461643

[30] Lebowitz, J., Rose, H., Speer, E.: Statistical mechanics of the nonlinear Schrödinger equation. J. Statist. Phys. 50, 657-687 (1988) Zbl 1084.82506 MR 0939505

[31] Miao, C., Wu, Y., Xu, G.: Global well-posedness for Schrödinger equation with derivative in $H^{1 / 2}(\mathbb{R})$. J. Differential Equations 251, 2164-2195 (2011) Zbl 1227.35236 MR 2823664

[32] Nahmod, A. R., Rey-Bellet, L., Sheffield, S., Staffilani, G.: Absolute continuity of weighted Wiener measures under gauge transformations. In preparation (2010)

[33] Oh, T.: Well-posedness theory of a one parameter family of coupled KdV-type systems and their invariant Gibbs measures. Ph.D. Thesis, Univ. of Massachusetts at Amherst (2007) MR 2711095

[34] Oh, T.: Invariant Gibbs measures and a.s. global well-posedness for coupled KdV systems. Differential Integral Equations 22, 637-668 (2009) Zbl pre05944836 MR 2532115

[35] Oh, T.: Invariance of the white noise for KdV. Comm. Math. Phys. 292, 217-236 (2009) (erratum in preparation) Zbl 1185.35257 MR 2540076

[36] Oh, T.: Invariance of the Gibbs measure for the Schrödinger-Benjamin-Ono system. SIAM J. Math. Anal. 41, 2207-2225 (2009) Zbl 1205.35268 MR 2579711 
[37] Ozawa, T.: On the nonlinear Schrödinger equations of derivative type. Indiana Univ. Math. J. 45, 137-163 (1996) Zbl 0859.35117 MR 1406687

[38] Rey-Bellet, L., Thomas L. E.: Low regularity solutions to a gently stochastic nonlinear wave equation in nonequilibrium statistical mechanics. Stoch. Process. Appl. 115, 1041-1059 (2005) Zbl 1083.82010 MR 2138813

[39] Sulem, C., Sulem, J. P.: The Nonlinear Schrödinger Equation. Self-Focusing and Wave Collapse. Appl. Math. Sci. 139, Springer, New York (1999) Zbl 0928.35157

[40] Takaoka, H.: Well-posedness for the one dimensional nonlinear Schrödinger equation with the derivative nonlinearity. Adv. Differential Equations 4, 561-580 (1999) Zbl 0951.35125 MR 1693278

[41] Takaoka, H.: Global well-posedness for Schrödinger equations with derivative in a nonlinear term and data in low order Sobolev spaces. Electron. J. Differential Equations 2001, no. 42, 23 pp. Z Zbl 0972.35140 MR 1836810

[42] Thomann, L., Tzvetkov, N.: Gibbs measure for the periodic derivative nonlinear Schrödinger equation. Nonlinearity 23, 2771-2791 (2010) Zbl 1204.35154 MR 2727169

[43] Tsutsumi, M., Fukuda, I.: On solutions of the derivative nonlinear Schrödinger equation II. Funkcial. Ekvac. 24, 85-94 (1981) Zbl 0491.35016 MR 0634894

[44] Tzvetkov, N.: Invariant measures for the nonlinear Schrödinger equation on the disc. Dynam. Partial Differential Equations 3, 111-160 (2006) MR 2227040

[45] Tzvetkov, N.: Invariant measures for the defocusing nonlinear Schrödinger equation. Ann. Inst. Fourier (Grenoble) 58, 2543-2604 (2008) Zbl 1171.35116 MR 2498359

[46] Tzvetkov, N.: Construction of a Gibbs measure associated to the periodic BenjaminOno equation. Probab. Theory Related Fields 146, 481-514 (2010) Zbl 1188.35183 MR 2574736

[47] Win, Y.: Global well-posedness of the derivative nonlinear Schrödinger equations on TT. Funkcial. Ekvac. 53, 51-88 (2010) Zbl 1194.35433 MR 2668514

[48] Zhidkov, P.: KdV and Nonlinear Schrödinger Equations: Qualitative Theory. Lecture Notes in Math. 1756, Berlin, Springer (2001) Zbl 0987.35001 MR 1831831 